\title{
Mechanistic Insights into Heterogeneous Catalytic Conversion of Light Oxygenates and Olefins from Biomass Derivatives
}

\author{
A dissertation presented to \\ the faculty of the school of engineering and applied science \\ University of Virginia
}

In fulfillment

of the requirement for the degree

of Doctor of Philosophy in Chemical Engineering

by

Erin Fitzharris

19 July 2017 


\begin{abstract}
The decreasing availability of fossil fuel resources has generated growing interests in chemical transformations of small oxygenates and olefin molecules derived from biomass sources into larger hydrocarbon fuel and chemical precursors. Aldol condensation of light oxygenates and alkene oligomerization of light olefins which proceed via C-C bond formation paths show potential for production of valuable chemical and fuel precursors. However, oxygenate coupling by $\mathrm{C}-\mathrm{O}$ bond formation such as in the reaction of methanol to dimethoxy methane (DMM) can occur together with C-C formation processes and lead to coke formation and catalyst deactivation. First principle density functional theory (DFT) calculations were carried out to probe the $\mathrm{C}-\mathrm{C}$ as well as $\mathrm{C}-\mathrm{O}$ coupling pathways over metal oxide as well as zeolite catalysts. More specifically we examined the paths for aldol condensation of aldehydes and ketones over $\mathrm{ZrO}_{2}$ and $\mathrm{ZnO}$ and alkene oligomerization and oxygenate coupling in zeolites. These three systems show similarities in the formation and stability of charged intermediates, stabilization of transition states, and intermolecular and catalytic steric effects on the activity for these reactions.
\end{abstract}

Aldol condensation of $\mathrm{C}_{2}-\mathrm{C}_{4}$ aldehydes and ketones over model $\mathrm{ZrO}_{2}$ and $\mathrm{ZnO}$ surfaces show that the rates are controlled by the kinetics for enolate formation, with $\mathrm{m}-\mathrm{ZrO}_{2}$ being the most active surface for aldol condensation. The rates of reaction in these systems were controlled by the stabilization of an adsorbed enolate that forms on the oxide where smaller aldehydes show higher interaction energies and thus faster rates of reaction.

Alkene oligomerization of $\mathrm{C}_{2}-\mathrm{C}_{4}$ olefins which proceed via similar $\mathrm{C}-\mathrm{C}$ bond formation steps in TON, MFI, and MOR zeolites show similar results, where the relative differences in stabilization of the reacting carbenium ion by the zeolite as well as differences in alkene adsorption largely determined the overall activity for alkene oligomerization in various zeolite structures. TON was predicted to be the most active catalyst as the narrow 1-D channel maximizes the number of potential secondary interactions with the carbenium ion at the transition state.

Oxygenate coupling reactions in zeolites lead to larger oxygenate species that can go on to form coke and deactivate the zeolite catalyst. The methanol to hydrocarbons process, for 
example, is inhibited by the formation of dimethoxy methane (DMM) formation. This reaction of methanol to DMM was calculated to be controlled by the low stability of the methyl cation that forms. The large size of the CHA cage as well as the exothermic nature of the proposed mechanism suggests that the CHA zeolite can accommodate further coupling reactions of much larger molecules that could ultimately block entrance into the open zeolite cages. 


\section{Table of Contents}

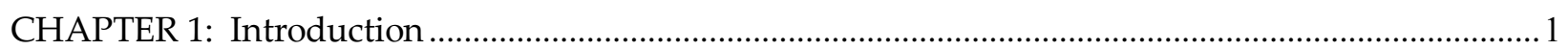

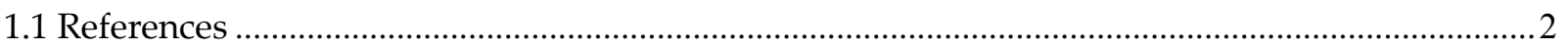

CHAPTER 2: Aldol Condensation in Metal Oxides................................................................................

2.1 Aldol Condensation for Light Oxygenates over Metal Oxides .................................................... 4

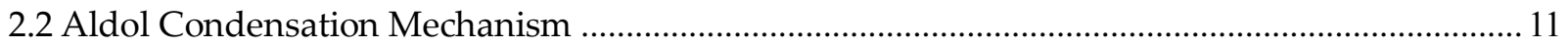

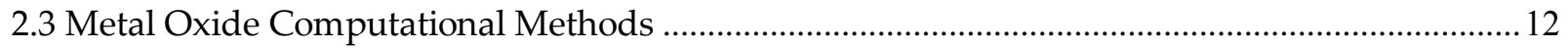

2.4 Calculation of Entropy for Free Energies of Activation and Adsorption .................................... 13

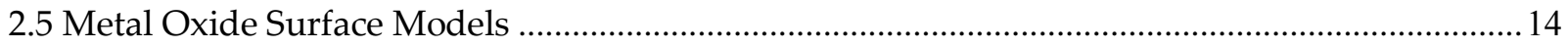

2.6 Gas Phase Deprotonation Energies of Light Aldehydes and Ketones........................................... 15

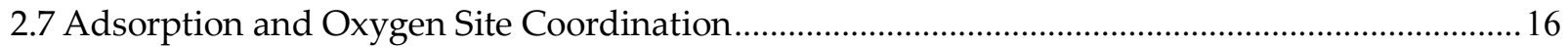

2.8 Adsorption of Aldehyde and Ketone Reactants onto Oxide Surfaces ......................................... 17

2.9 Intrinsic Energies for Enolate Formation Reaction on Oxide Surfaces........................................ 18

2.10 Apparent Activation Energies and Gibbs Free Activation Energies for Enolate Formation .....23

2.11 Born-Haber Cycle Analysis and Effect of Catalyst-Intermediate Interaction.............................25

2.12 C-C Bond Formation Step in Aldol Condensation .......................................................................29

2.13 Comparison of C-C Bond Formation and Enolate Formation Apparent Activation Energy .... 32

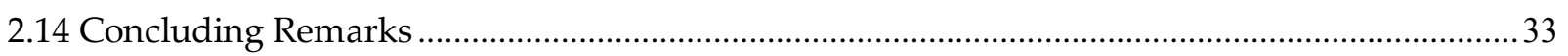

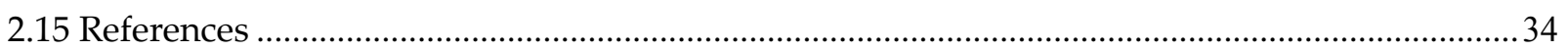

CHAPTER 3: Alkene Oligomerization in Zeolites ............................................................................... 38

3.1 Alkene Oligomerization of Light Olefins within Zeolites ......................................................... 38

3.2 Catalytic Mechanism for Alkene Oligomerization in Zeolites ......................................................42

3.3 Alkene Oligomerization Computational Methods ........................................................................... 43

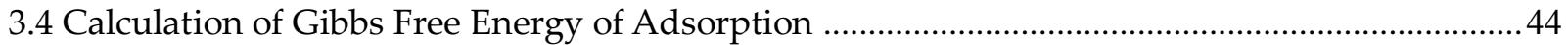

3.5 Calculation of Entropy for Free Energies of Activation ................................................................. 45

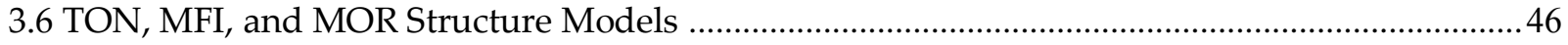

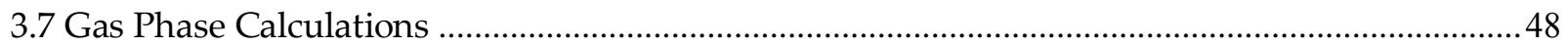

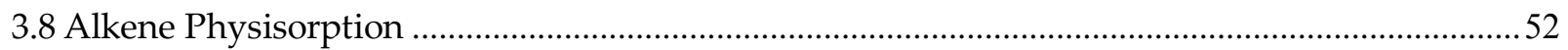

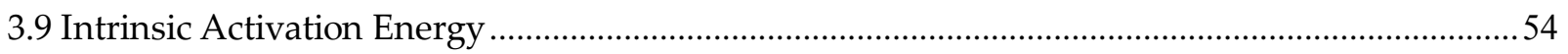

3.10 Charge analysis of Alkene Oligomerization in Zeolites ............................................................55

3.11 Close-contact Analysis and Van der Waals Volume Overlap ....................................................... 65

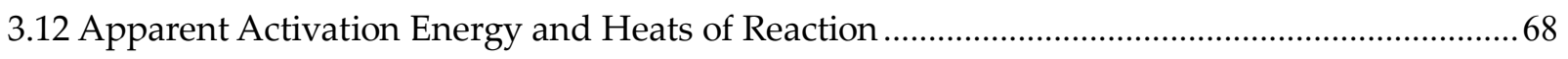


3.13 Apparent Gibbs Free Energy of Activation .............................................................................. 71

3.14 Born Haber Cycle Analysis and Interaction Energy ….............................................................. 73

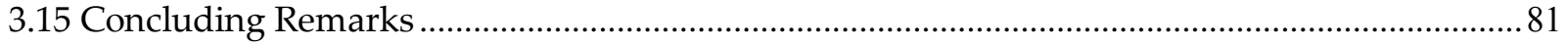

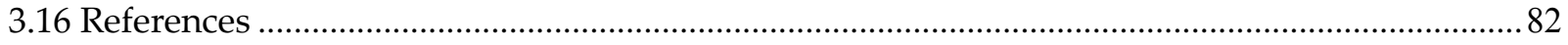

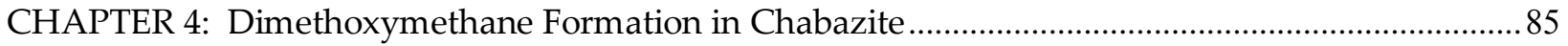

4.1 Deactivation during Methanol-to-Olefins Reaction and Dimethoxymethane Formation in CHA

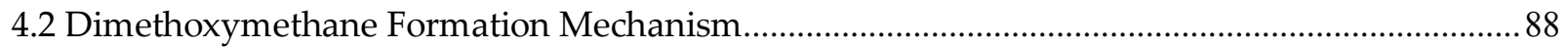

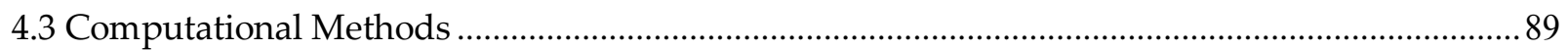

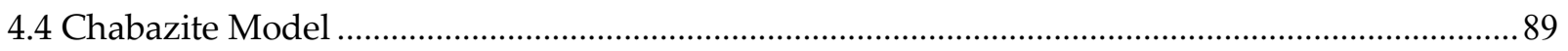

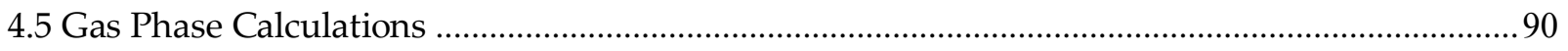

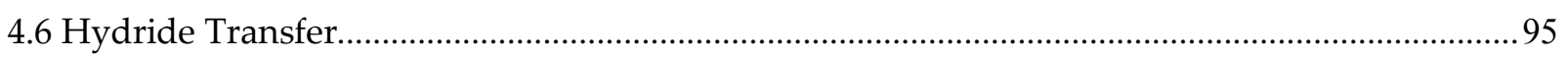

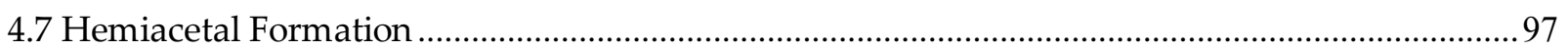

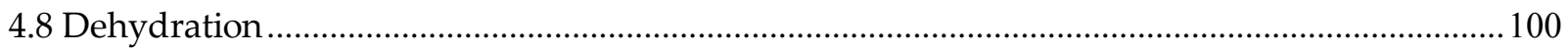

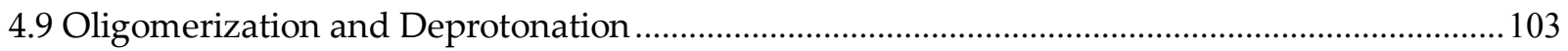

4.10 Apparent Energies of Activation for DMM Formation in CHA ........................................... 106

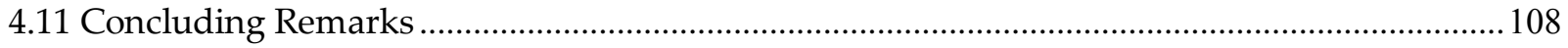

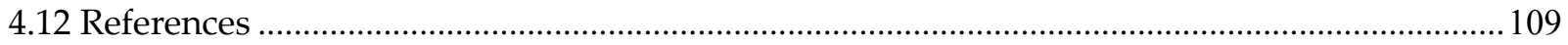

CHAPTER 5: Conclusions on the Similarities Between Aldol Condensation, Alkene

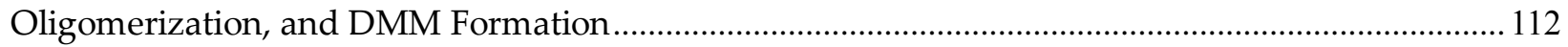

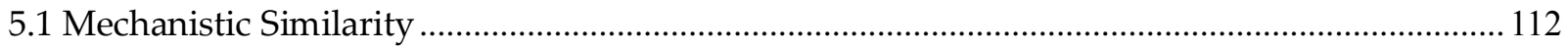

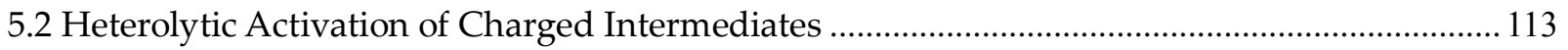

5.3 Delocalization and Steric Limitations of Electrophilic/Nucleophilic Reactant.......................... 114

5.4 Transition State Structure and Energetic Barriers Associated with Ion Formation .................... 117

5.5 The Role of the Catalyst on Intermediate Stabilization .............................................................. 119

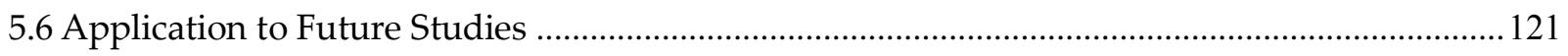

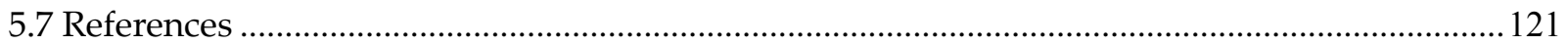




\section{CHAPTER 1: Introduction}

Fossil fuels are presently the main source of carbon-based products used in the production of heat and power. The combustion of fossil fuels and their derivatives, however, has been associated with a steady increase in greenhouse gas levels worldwide [1], and has been implicated in climate change [2]. A more attractive solution to the world's energy needs would draw upon multiple renewable resources and prevent greenhouse gas emissions. Biomassderived fuels and products are well suited to begin to meet these needs. They are renewable, readily available, and $\mathrm{CO}_{2}$-neutral, as the $\mathrm{CO}_{2}$ that is produced during combustion can be taken up by green plants and algae during photosynthesis [3]. There are different possible sources of biomass that could be used as raw material for production of biofuels and chemicals, including waste materials, forest products, energy crops, or aquatic biomass [4]. Similar to the petrochemical industry, where crude oil is fractionated and refined to produce varying types of fuels and specialty chemicals, biomass could be used in a bio-refining process to achieve similar results. However, biomass-derived molecules are small molecules that have an abundance of oxygen and therefore, any process used to produce biofuels and specialty chemicals must create C-C bonds to form longer hydrocarbon chains and reduce the oxygen/carbon ratio from the biomass-derived intermediates [1].

Aldol condensation provides an attractive route for the upgrading of light oxygenates to biofuels as the reaction increases the carbon chain length via $C^{*}-C$ bond formation and simultaneously reduces the oxygen/carbon ratio through dehydration. Alkene oligomerization also shows potential for catalytic conversion of light alkenes into longer hydrocarbon chains for use in chemicals or biofuels. At the same time, the coupling of oxygenates by $\mathrm{C}^{*}-\mathrm{O}$ bond formation, such as dimethoxymethane (DMM) formation, occurs alongside these processes and is one of the many reactions thought responsible for the eventual deactivation of catalysts through the formation of large coke molecules that block potential catalyst sites. Understanding these mechanisms at a molecular level grants insight into the catalytic features that promote $\mathrm{C}^{*}$ $\mathrm{C}$ bond formation necessary for the upgrading of biomass-derived molecules, and also gives insight into the features responsible for $\mathrm{C}^{*}$-O bond formation that leads to catalyst deactivation. 
Each of these catalytic reactions involve the formation of a charged intermediate species that then undergoes $\mathrm{C}^{*}-\mathrm{C}$ bond formation or $\mathrm{C}^{*}$-O bond formation with a second molecule. The goals of this work for each of these catalytic reactions were to better understand how the charged intermediate is formed, to identify what factors influence the bond formation of said charged intermediate with a second reactant, analyze which features of the catalyst and reactants facilitate both these steps, and then summarize their overall effects on the kinetics of the reaction. First principle density functional theory (DFT) calculations were carried out herein to examine the energetics of each catalytic mechanism and potentially quantify the different reactant and catalyst features on observed differences in activity. Charge analyses were used to identify the nature of the charged intermediate formation step by observing the transfer of charge between the intermediates and the catalyst along the reaction coordinate. Born-Haber cycle analyses were also used to isolate the fundamental thermochemical steps that make up the activation energies of the rate determining steps, while also quantifying the stabilization provided by the catalyst on the transition state.

Using first principle density functional theory (DFT) calculations, the work herein analyzes and investigates aldol condensation, alkene oligomerization, and DMM formation for the coupling of light oxygenates and alkene intermediates derived from biomass into longer hydrocarbon chains.

\subsection{References}

[1] Huber, G. W.; Iborra, S.; Corma, A., “Synthesis of transportation fuels from biomass: Chemistry, catalysts, and engineering", Chem. Rev. 2006, 106, 4044- 4098.

[2] Schimel, D. "Contributions to accelerating atmospheric $\mathrm{CO}_{2}$ growth from economic activity, carbon intensity, and efficiency of natural sinks", Proceedings of the National Academy of Sciences 2007, 104 (47), 18353-18354.

[3] Wyman, C.E.; Hinman, N.D., “Ethanol: Fundamentals of production from renewable feedstocks and use as a transportation fuel", Appl. Biochem. Biotechnol. 1990, 24/25, 735753. 
[4] U.S. Department of Energy, Feedstock Composition Gallery, Washington, DC 2005, http://www.eere.energy.gov/biomass/feedstock_glossary.html. 


\section{CHAPTER 2: Aldol Condensation in Metal Oxides}

\subsection{Aldol Condensation for Light Oxygenates over Metal Oxides}

Aldol condensation over heterogeneous catalysts shows high potential for the transformation of small biomass derivatives through the coupling of small molecule oxygenates and subsequent dehydration. The reaction can proceed by base-catalyzed or acid-catalyzed routes, via abstraction of the proton off the carbon alpha to the carbonyl to form the enolate ion by a basic catalyst, or through simultaneous protonation of the carbonyl oxygen and deprotonation of the alpha carbon to form the enol intermediate by an acidic catalyst. The reaction continues with subsequent $\mathrm{C}-\mathrm{C}$ bond formation of the nucleophilic intermediate to a second oxygenate followed by dehydration to yield the final aldol condensation products with both increased carbon chain length and decreased oxygen/carbon ratio. Base-catalyzed aldol condensation is distinguished from acid-catalyzed condensation by the first two steps of the reaction: the formation of a charged intermediate via enolate formation from an aldehyde or ketone starting material, and C-C coupling between the enolate's nucleophilic $\alpha$-carbon and the weakly electrophilic carbonyl carbon center of a neighboring aldehyde or ketone (Figure 2.2.1).

The first step which involves the formation of the enolate is thought to proceed via the activation of the acidic $\mathrm{C}_{\alpha}-\mathrm{H}$ bond. Enolate formation is dictated by the gas phase stability of the enolate and its stabilization on the acid sites on the catalyst surface. Using pulsed ionization high pressure mass spectroscopy, Burkell et al. [1] determined the gas phase acidities of $\mathrm{C}_{3}-\mathrm{C}_{10}$ 2-ketones and showed a clear increase in gas phase acidity with chain length up to $\mathrm{C}_{10}$ along with a higher enolate stability at the secondary $\alpha$-carbon position with respect to the primary $\alpha$ carbon position. An earlier study by Chyall et al. [2] reported the relative enolate stabilities for $\mathrm{C}_{4}-\mathrm{C}_{6} 2$-ketones of: $2^{\circ}>1^{\circ}>3^{\circ}$ via fluoride deprotonation reactions. The results are consistent with those by Burkell et al. They also noted the strong stabilizing effects of the solvent, counterion, and associated aggregates during enolate reaction, and referred to such effects as "indispensable elements" to the deprotonation of asymmetric ketones. Such effects will also likely be important in understanding the enolate formation paths on solid acid and base catalysts. 
Enolate formation occurs over heterogenous catalysts after adsorption of the oxygenates upon the surface, and the modes of binding for aldehydes during aldol condensation on solid catalysts have been investigated. Finklestein-Shapiro et al. analyzed the adsorption of acetaldehyde onto $\mathrm{TiO}_{2}$ nanorods at low pressures using chemical ionization mass spectrometry as well as Fourier transform infrared (FT-IR) spectroscopy [3]. They detected reversible adsorption of acetaldehyde with an energy of $44 \mathrm{~kJ} / \mathrm{mol}$, consistent with multiple hydrogen bonds, and trace aldol condensation reactions with oxidative binding to the surface. Raskó, Keskés, and Kiss analyzed acetaldehyde interaction with $\mathrm{TiO}_{2}$-supported $\mathrm{Pt}, \mathrm{Rh}$, and $\mathrm{Au}$ catalysts using FT-IR and mass spectrometry [4] and identified two modes of acetaldehyde adsorption on $\mathrm{TiO}_{2}$. The first is through a strong bond that results from the donation of the lone pair of electrons on the oxygen of acetaldehyde to the Ti Lewis acid site. The second mode of adsorption involves a weak hydrogen bonding interaction between the oxygen on the aldehyde and the protic hydrogens on the hydroxyl groups on the $\mathrm{TiO}_{2}$ surface. While the metal that was present enhanced the formation of $\mathrm{H}_{2}$ and dehydration, the aldolization reaction predominantly proceeded via the activation and coupling of strongly coordinated aldehydes bound to the $\mathrm{Ti}$ sites on the $\mathrm{TiO}_{2}$ surface.

More detailed kinetics along with insights into the mechanisms involving enolate as well as $\mathrm{C}-\mathrm{C}$ bond formation have been reported for both homogeneous as well as heterogeneous base-catalyzed reactions. Singh et al. analyzed the surface intermediates of acetaldehyde aldol condensation on Degussa P-25 $\mathrm{TiO}_{2}$ using IR spectroscopy at low temperatures [5] and clearly showed $\mathrm{Ti}-\mathrm{O}$ stretching bands that form during acetaldehyde adsorption, consistent with adsorption of the acetaldehyde oxygen at the Lewis acid metal site. Gradual increase in temperature resulted in growth of IR features for a negatively charged carbanion and 3hydroxybutanal surface species, consistent with formation of enolate and aldol intermediates from base-catalyzed aldol condensation. West et al. [6] examined the homogeneous aldol condensation of furfurals and ketones using $\mathrm{NaOH}$ in a biphasic system and showed that the rates were first-order in the aldehyde. More detailed analyses showed that the reagent ratios correlated to the selectivity for single condensation and multi-condensation type products, whereas the $\mathrm{NaOH}$-furfural ratio greatly determined the rate of condensation. Rekoske and 
Barteau analyzed the kinetics, selectivity and deactivation of acetaldehyde aldol condensation on oxidized anatase $\mathrm{TiO}_{2}$ [7]. They noted a significant deactivation within the first few minutes on stream. The activity for aldol condensation following the initial deactivation, however, remained fairly constant demonstrating first-order kinetics for acetaldehyde consumption and high selectivities for aldol condensation products at low pressures. All of the heterogeneous and homogeneous studies discussed here showed first-order kinetics at low pressures for acetaldehyde consumption, which involves bi-molecular C-C bond formation steps. This would suggest that the kinetically relevant step is either enolate formation or adsorption, as any step at or following C-C bond formation would result in second-order kinetics at low pressures.

Several studies reported in the literature illustrated the sensitivity of aldol condensation on reduced and oxidized surfaces, and discuss the importance of Lewis base-site strength. Idriss, Kim, and Barteau studied aldol condensation and competing reactions of acetaldehyde on single crystal rutile $\mathrm{TiO}_{2}$ carried out at ultra-high vacuum and powdered anatase $\mathrm{TiO}_{2}$ at 1 atm. They observed activity for aldol condensation in both systems, with competing reactions including reduction of aldehydes to alcohols, Cannizaaro reaction, and minor reductive coupling to form butane [8]. While the selectivities and yields to form crotonaldehyde varied across systems, it still remained a major product and aldol condensation was suggested to be relatively structure-insensitive. This was contrasted to carboxylate ketonization, which is another bi-molecular reaction that instead has high structure sensitivity due to the large catalytic ensemble required. A follow up study by Idriss and Barteau [9] analyzed shifts in selectivity for acetaldehyde aldol condensation on oxidized and reduced $\mathrm{TiO}_{2}$ single-crystals and showed higher aldol condensation selectivities on oxidized surfaces along with an increased number of Lewis base sites. Aldol condensation activity which was still observed on the reduced surfaces was suggested to be less sensitive to surface reconstruction. Idriss et al. [10] found that $\mathrm{Pd}$ and $\mathrm{Co}$ supported systems reduced the $\mathrm{CeO}_{2}$ surface, and resulted in lower aldol condensation activities and selectivities than those on pure $\mathrm{CeO}_{2}$ surface. While not as selective as $\mathrm{TiO}_{2}$ or $\mathrm{Cu} / \mathrm{ZnO} / \mathrm{Al}_{2} \mathrm{O}_{3}$, the pure $\mathrm{CeO}_{2}$ surface produced a $48 \%$ selectivity to single aldol condensation products. Raskó and Kiss analyzed the adsorption and reactions of acetaldehyde on Degussa P-25 $\mathrm{TiO}_{2}, \mathrm{CeO}_{2}$, and $\mathrm{Al}_{2} \mathrm{O}_{3}$ for both $\mathrm{O}_{2}$-pretreated and $\mathrm{H}_{2}$-pretreated 
surfaces [11] and showed that the reduced surfaces predominantly carried out aldol condensation to form crotonaldehyde. The oxidized surfaces, on the other hand, resulted in higher concentrations of benzene which is thought to be produced via an intramolecular cyclization of a double aldol condensation product between acetaldehyde and crotonaldehyde. Zhang, Hattori, and Tanabe studied aldol addition of butanal over alkaline earth oxides, $\mathrm{ZrO}_{2}$, and $\mathrm{La}_{2} \mathrm{O}_{3}$, to understand the active site and its effect on the activity for aldol addition in batch reactor at $0{ }^{\circ} \mathrm{C}[12]$. At this low temperature, the activity greatly depended upon the basic strength of the catalyst whereby poisoning by $\mathrm{CO}_{2}$ and $\mathrm{H}_{2} \mathrm{O}$ decreased activity for aldol condensation by almost half. While pyridine demonstrated some poisoning, it was much less than that for $\mathrm{CO}_{2}$ which suggested a higher sensitivity of the rate of condensation to the base sites over the acid sites.

Other studies on $\mathrm{TiO}_{2}$ and other oxides report a different picture suggesting that the Lewis acid sites dominate aldol condensation. Luo and Falconer examined the condensation of acetaldehyde over Degussa $\mathrm{P}-25 \mathrm{TiO}_{2}$ [13] and showed the formation of single and double condensation products, as well as dehydro-cyclization side products thought to be responsible for $\mathrm{TiO}_{2}$ deactivation at elevated temperatures. In comparison to anatase, $\mathrm{P}-25 \mathrm{TiO}_{2}$ has a higher concentration of acid sites and adsorbed acetaldehyde, which was suspected to facilitate the formation of both double-condensation products and their dehydro-cyclization derivatives. Similar conclusions were also reached by Ordomsky, Sushkevich, and Ivanova, who studied acetaldehyde aldol condensation over silica supported magnesia and zirconia catalysts and used IR spectroscopy of $\mathrm{CO}_{2}$ and pyridine adsorption as well as TPD of $\mathrm{NH}_{3}$ and $\mathrm{CO}_{2}$ to explore the role of acid and base sites for aldol condensation [14]. Co-feeding of $\mathrm{CO}_{2}$ to purposefully poison Lewis base sites did not inhibit the initial acetaldehyde conversion rates for either $\mathrm{MgO}$ or $\mathrm{ZrO}_{2}$. The co-feeding of pyridine, on the other hand, appeared to poison Lewis acid sites which significantly reduced the catalyst activity for condensation. IR spectroscopy of pyridine poisoned and non-poisoned surfaces showed a large decrease in amount of acetaldehyde adsorbed, and concluded that the Lewis acid sites were responsible for the catalytic activity of both $\mathrm{ZrO}_{2}$ and $\mathrm{MgO}$ for aldol condensation. These results signifying the importance of acid sites for aldol condensation and the sensitivity which is rather different than the results on the 
reduced surfaces, which show the importance of base site strength and the general structureinsensitivity of the reaction towards the catalytic surface.

Many of the elementary steps in aldol condensation require Lewis acid and base site pairs, and as such, both are likely important. Tanabe and Hölderich surveyed 127 processes and 180 acid-base catalysts used in existing industrial processes [15]. Fourteen of these are solid bifunctional catalysts with weak acid-base strength and exhibit surprisingly high activity and resistance to coking. A review on the future of acid-base catalysis in industry stressed the need for acid as well as base catalyzed reactions to be tested over weak acid-base bifunctional catalysts as their activity has been noted as particularly high due to the close orientation and coordination of base and acid sites [16]. Of the solid bifunctional catalysts discussed, Tanabe and Yamaguchi analyzed the acid-base bifuntionality of $\mathrm{ZrO}_{2}$ and its catalytic activity for acidbase type mechanisms [17]. A TPD analysis of the $\mathrm{ZrO}_{2}$ acid-base sites concluded that $\mathrm{ZrO}_{2}$ is nearly a neutral catalyst, with the acid-site strength found to be as comparably weak as the base-site strength. Even with the relative weak site strength, several reactions including $\mathrm{C}-\mathrm{H}$ bond cleavage, ketone synthesis, and $\alpha$-olefin synthesis from alcohols were all found to produce relatively high yields and selectivities on $\mathrm{ZrO}_{2}$ catalysts in comparison to more basic or more acidic catalysts. The activity for these reactions was suggested to derive from the weak strength of both acids and bases that facilitate the heterolytic activation of surface intermediates while limiting the binding and deactivation of these intermediates that form. It was also noted that the orientation of acid-base pairs can be significantly altered by catalyst preparation, pretreatment conditions, and the addition of other metal-oxides. The specific location and orientation of the acid-base sites can be important in the activation of particular molecules and can potentially be explored through the adsorption of acid/base probe molecules [18]. Climent et al. analyzed Knovenagel condensation, aldolization, and Michael addition over catalysts with different acid and base site strengths including $\mathrm{MgO}$, calcined hydrotalcites, and amorphous aluminophosphates (ALPO) in an effort to explore the importance of acid and base functionality on catalysis [19]. Of the catalysts analyzed, ALPO catalysts exhibited weak acid-base site strength, but produced relatively high yields and high selectivities in comparison to more basic solid catalysts. They concluded that the high activity may derive from the cooperative role of 
milder acid and base sites. These studies show the potential of weaker acid-base bifunctional catalysts to excel in base-catalyzed reactions that were otherwise dominated by solid base catalysts.

More recent studies for aldol condensation activity highlight the role of cooperativity between the acid-base sites in driving aldol condensation, particularly in those catalysts with weak acid-base site pairs such as $\mathrm{ZrO}_{2}$. Sad et al. analyzed C-C and C-O formation pathways for condensation of alkanols and alkanals over oxide-supported copper catalysts and show the surprising results that $\mathrm{Cu}$ metal can catalyze aldol condensation [20]. They show that $\mathrm{Cu}$ catalyzes the interconversion of alkanols and alkanals, and as such the formation of adsorbed alkoxides act as a base for deprotonation of propanal and enolate formation. The basic alkoxide sites are formed catalytically during the reaction and work together with the metal $\mathrm{Cu}$ sites that act as Lewis acid sites to catalyze the aldol condensation and esterification. Tai and Davis studied the cross aldol condensation of propionic acid with formaldehyde over $\mathrm{MgO}, \mathrm{SiO}_{2}$, $\mathrm{Al}_{2} \mathrm{O}_{3}$, and $\mathrm{ZrO}_{2}$ catalysts while utilizing stepwise TPD of $\mathrm{NH}_{3}$ and $\mathrm{CO}_{2}$ to analyze the catalyst site properties [21]. Their TPD analysis found that of those catalysts tested, only $\mathrm{Al}_{2} \mathrm{O}_{3}$ and $\mathrm{ZrO}_{2}$ possessed a significant number of both acid and base sites. Comparison of the activity for aldol condensation found that $\mathrm{MgO}$, the classic basic oxide, was completely inactive for aldol condensation whereas $\mathrm{ZrO}_{2}$ showed relatively good activity for aldol condensation as well as high water resistance that otherwise greatly reduced the activity for aldol condensation, especially for those catalysts with loaded cesium. These experimental findings in addition to Tanabe's conclusions on acid-base bifunctionality illustrate the potential of $\mathrm{ZrO}_{2}$ and $\mathrm{ZnO}$ surfaces for aldol condensation through the oddly high activity on weak acid-base site pairs.

Recent experimental evidence, however, has found high deactivation due to strongly bound aldol products upon the surface. Despite the relatively weak strength of the acid and base site pairs, strong product binding greatly affects the observed kinetics on metal oxides. Work by Young et al. [22] analyzed the aldol condensation of acetaldehyde over pure anatase $\mathrm{TiO}_{2}$ surfaces and found very high deactivation within the first hour on stream. A comparison of reaction rates with time on stream yielded deactivation curves similar to the deactivation of acetaldehyde condensation on Degussa P-25 $\mathrm{TiO}_{2}$ discussed by Rekoske et al. [7]. Only at low 
acetaldehyde partial pressures of less than $0.4 \mathrm{kPa}$ was no deactivation detected, and the reaction was found to be first order with respect to acetaldehyde pressure. However, at these low pressure conditions, Young et al. found no kinetic isotope effect, indicating that the enolate formation step is not kinetically relevant under these conditions. Rather, the acetaldehyde adsorption and crotonaldehyde product desorption steps on pure $\mathrm{TiO}_{2}$ appeared to be the kinetically relevant steps. This would both satisfy the first-order dependence on acetaldehyde at low pressures, and the relative dependence of product desorption due to site availability that yields a zero order dependence on acetaldehyde at high acetaldehyde pressures.

However, experimental work by Wang et al. [23] found that, when $\mathrm{TiO}_{2}$ is accompanied by $\mathrm{H}_{2}$ gas and a supported $\mathrm{Cu} / \mathrm{SiO}_{2}$ co-catalyst, deactivation due to strongly bound products is eliminated. A comparison of condensation rates with time on stream for a pure $\mathrm{TiO}_{2}$ catalyst yielded the same deactivation curves as discussed by Young et al. [22] and Rekoske et al. [7]. A comparison of condensation rates with time on stream for a $\mathrm{TiO}_{2}$ catalyst with $\mathrm{H}_{2}$ gas and supported metal co-catalyst showed no deactivation with time on stream. The presence of $\mathrm{H}_{2}$ gas and the $\mathrm{Cu} / \mathrm{SiO}_{2}$ catalyst were suggested to act as hydrogenating agents that saturated the aldol condensation products as soon as they desorbed off the $\mathrm{TiO}_{2}$ surface. This would decrease the binding of the products on the $\mathrm{TiO}_{2}$ surface and simultaneously decrease the crotonaldehyde concentration in the gas phase. These two effects would increase the rate of desorption of crotonaldehyde and therefore decrease the effect of deactivation from product binding. Under these conditions, a kinetic isotope effect using deuterated acetone was detected for $\mathrm{TiO}_{2}$, signifying that enolate formation is kinetically relevant for acetaldehyde aldol condensation on $\mathrm{TiO}_{2}$ in the presence of $\mathrm{H}_{2}$ gas and a $\mathrm{Cu} / \mathrm{SiO}_{2}$ co-catalyst. The combination of observed kinetics by Young et al. and Wang et al. suggests that, at product coverages, the reaction depends upon the molecules' ability to get on and off the surface whereas under low product coverages, the reaction instead depends upon the metal oxide's inherent ability to facilitate the aldol condensation reaction. The work herein analyzes the effects of reactants and metal oxide surfaces for aldol condensation at ideal non-covered surface so as to isolate the inherent activity of these systems without the effects of deactivation. 
The large body of work concerning aldol condensation cover different metal oxide catalysts discussed above clearly show that the site strength, location, and proximity of the acid and base site pairs on metal oxide surfaces control the catalytic activity and selectivity for these as well as other reactions. Little, however, is known about the detailed atomic structure of the active acid and base sites, and the cooperation between these sites in controlling the molecular transformations that occur during aldol condensation. First principle density functional theory (DFT) calculations were carried out herein to examine the sites and the mechanism that carry out aldol condensation and, more specifically, the enolate formation and C-C bond formation elementary steps over model zirconia and zinc oxide surfaces to help identify the kinetically relevant features of the catalyst and the reactants.

\subsection{Aldol Condensation Mechanism}

Base-catalyzed aldol condensation over metal oxides is hypothesized to proceed via adsorption of two aldehydes or ketones via adsorption of the carbonyl oxygen onto the metal Lewis acid sites (Figure 2.2.1).

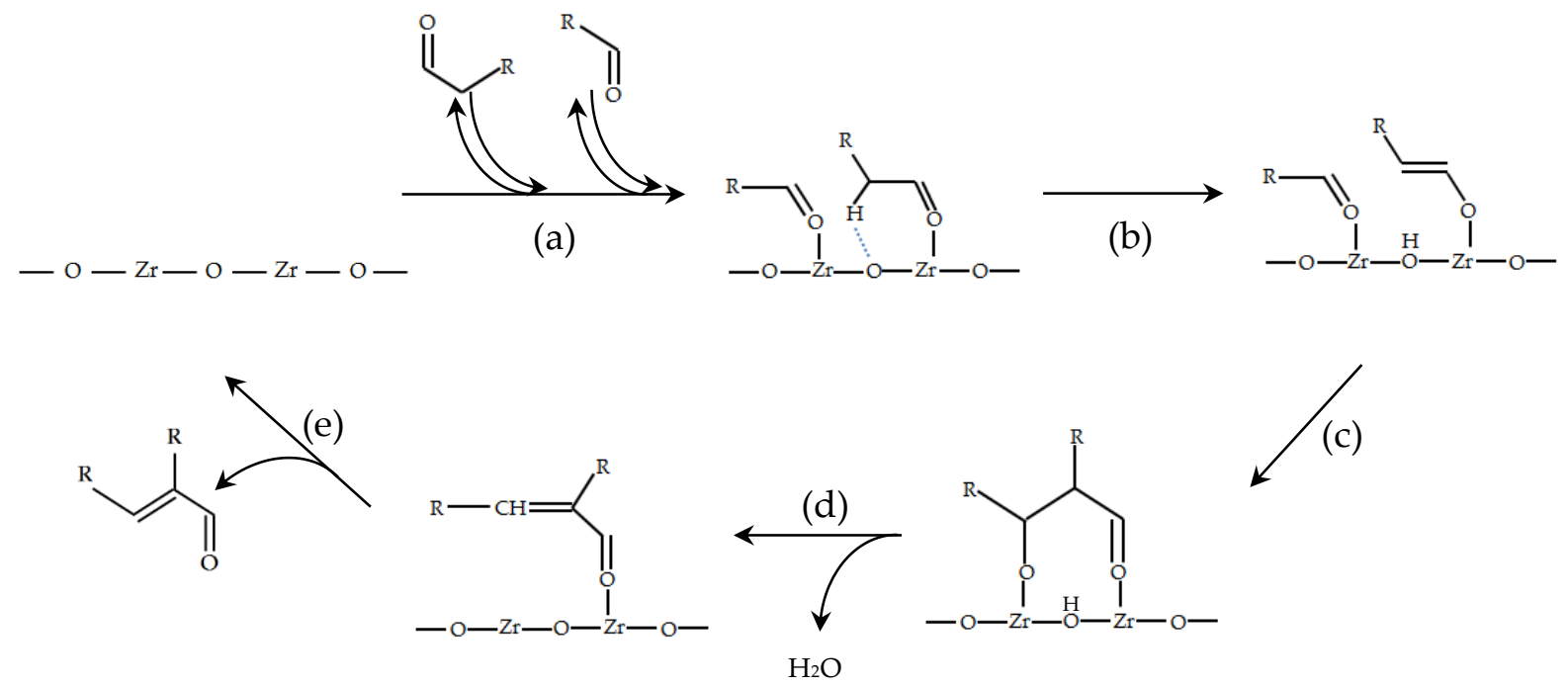

Figure 2.2.1: Reaction scheme for the proposed elementary reactions involved in aldol condensation over model zirconia surfaces. The steps include: (a) oxygenate adsorption, (b) enolate formation, (c) C-C bond formation, (d) dehydration, (e) enone product desorption. 
The $\alpha$-proton of one of the oxygenates is deprotonated by the neighboring oxygen Lewis base site to form the surface enolate, which was experimentally determined by Singh et al. as an active surface intermediate by low temperature IR spectroscopy [5]. The enolate then forms a $\mathrm{C}-\mathrm{C}^{*}$ bond to the carbonyl carbon of the second adsorbed oxygenate via nucleophilic backside attack and forms the surface aldol intermediate. Re-protonation and intramolecular hydride shift leading to subsequent dehydration forms water and the final aldol condensation product as a larger conjugated aldehyde or ketone, depending on the reactants.

\subsection{Metal Oxide Computational Methods}

All of the calculations reported herein were carried out using periodic dispersion corrected density functional theory (DFT-D2) as implemented in the Vienna ab initio Simulation Package (VASP) [24]. The effects of dispersion were accounted for through the use of semiempirical Grimme-D2 corrections [25]. The Perdew-Burke-Ernzerhof (PBE) exchangecorrelation functional was used to determine the nonlocal gradient corrections to exchange and correlation energies. Projector-augmented wave (PAW) pseudopotentials were used to describe core electrons [26]. The valence electrons were described using a plane-wave basis set with an energy cutoff of $396 \mathrm{eV}$. A Monkhorst-Pack k-point mesh of $(3 \times 3 \times 1)$ was used to sample the first Brillion zone. All of the calculations were carried out spin polarized. The wave functions and charge density were converged to within $10^{-6} \mathrm{eV}$. Atomic charges were analyzed using the Bader charge analysis which describes the charge distribution by partitioning of the electron density [27]. The saddle points were initially isolated using the nudged elastic band method (NEB) to map out the reaction coordinate [28, 29]. Sixteen evenly spaced points were chosen along the reaction coordinate and the structures at each of these points were minimized until the forces perpendicular to the reaction path converged to within $0.25 \mathrm{eV} / \AA$. The minimum energy reaction path and the approximate transition states were subsequently used as input to isolating more refined transition states using the dimer method [29]. The dimer calculations were converged until the forces perpendicular to the reaction mode were less than $0.05 \mathrm{eV} / \AA$.

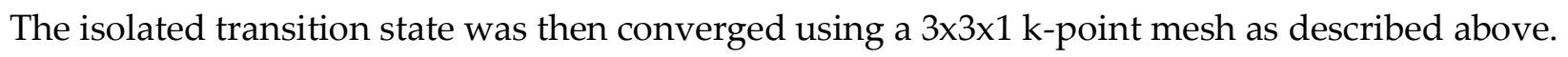




\subsection{Calculation of Entropy for Free Energies of Activation and Adsorption}

The entropy of adsorption was calculated using the vibrational contributions to entropy for the metal oxide systems with an adsorbed aldehyde and then compared to the entropy of the intrinsic metal oxide system with a bare metal oxide surface and gas phase molecule. The entropy of the reference state consisting of a bare metal oxide surface and gas phase aldehyde was measured using vibrational frequencies of the bare metal oxide surface as well as translational, rotational, and vibrational contributions to entropy for the gas phase aldehyde.

The vibrational partition function was calculated using the first vibrational mode as reference:

$$
q_{V}=1 /\left(1-\exp \left(\frac{h c v}{k_{B} T}\right)\right)
$$

The vibrational, translational, and rotational contributions to the partition function of the aldehyde gas molecule $\left(Q_{\text {gas }}\right)$ were calculated using ideal gas partition function expressions at $1 \mathrm{~atm}$ and 298.15 Kelvin, where $m_{\text {gas }}$ is the mass of the aldehyde and $V_{\text {gas }}$ is the volume of the ideal gas.

$$
Q_{g a s}=q_{g a s}=V_{g a s} *\left(\frac{2 \pi m_{g a s} k_{B} T}{h^{2}}\right)^{3 / 2}
$$

The change in entropy of adsorption $\left(\Delta S_{\text {ads }}\right)$ was then calculated via the following equations, where $Q_{v}$ is the vibrational partition function of the metal oxide with an adsorbed aldehyde, $Q v, R$ is the vibrational partition function of the naked metal oxide surface at the reference state, and $\mathrm{Q}_{\text {gas }}$ is the partition function of the aldehyde gas molecule.

$$
\begin{gathered}
Q_{V}=\prod q_{V} \\
S=R \ln \left(Q_{V}\right) \\
\Delta S_{a d s}=R \ln \left(\frac{Q_{V}}{Q_{V, R} Q_{g a s}}\right)
\end{gathered}
$$

The entropy of reaction was calculated in a similar manner, in which the vibrational partition function of the metal oxide system at the enolate transition state was compared to the partition functions of the metal oxide surface and gas phase aldehyde. 


$$
\Delta S_{a p p}^{\neq}=R \ln \left(\frac{Q_{V}^{\neq}}{Q_{V, R} Q_{g a s}}\right)
$$

With the entropy of reaction and the entropy of adsorption, the Gibbs free energies of reaction $\left(\Delta \mathrm{G}^{\neq}\right)$and Gibbs free energies of adsorption ( $\left.\Delta \mathrm{Gads}\right)$ was calculated using the following equation at $\mathrm{T}=523 \mathrm{~K}$.

$$
\Delta G^{\neq}=\Delta H^{\neq}-T \Delta S^{\neq}
$$

\subsection{Metal Oxide Surface Models}

The calculations reported herein examine the monoclinic $\mathrm{ZrO}_{2}\left(\begin{array}{lll}-1 & 1 & 1\end{array}\right)\left(\mathrm{m}-\mathrm{ZrO}_{2}\right)$, the

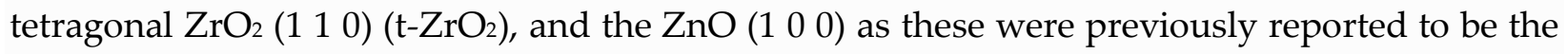
most stable surface structures [30-32] (Figure 2.5.1). Each surface was constructed to have a consistent number of metal sites available for reactant adsorption rather than consistent number of oxygen sites as the number of oxygen sites per until cell varies between catalysts. The unit cells were comprised of a metal oxide slab and a $10 \AA$ vacuum layer to separate each slab to reduce interactions with mirror images. The metal oxide slab consisted of 4 metal layers for $\mathrm{ZnO}$ and $\mathrm{m}-\mathrm{ZrO}_{2}$ surfaces, and 3 metal layers for the $\mathrm{t}-\mathrm{ZrO}_{2}$ surface. The monoclinic (-111) $\mathrm{m}-$ $\mathrm{ZrO} 2$ surface used was constructed using a (2x1) unit supercell with $4 \mathrm{~m}$ - $\mathrm{ZrO} 2$ layers, creating a total slab thickness at $14 \AA$. The m-ZrO2 model surface has 2 possible bridge oxygen sites and 8 possible metal sites for reactant adsorption. The tetragonal $\mathrm{t}-\mathrm{ZrO} 2$ surface was constructed using a (2x1) unit supercell with $3 \mathrm{t}-\mathrm{ZrO} 2$ layers, creating a total slab thickness at $10 \AA$. The $\mathrm{t}$ $\mathrm{ZrO} 2$ surface has 6 possible tri-coordinated oxygen sites and 8 possible metal sites for reactant adsorption. The $\mathrm{ZnO}$ surface model was comprised of $(2 \times 2)$ unit supercell with a $10 \AA$ vacuum slab above and approximately $4 \mathrm{ZnO}$ layers, creating a total slab thickness at $11 \AA$. With this model, the $\mathrm{ZnO}$ surface has 4 possible tri-coordinated oxygen sites and 8 possible metal sites for reactant adsorption. The atoms in the bottom 2 layers for $\mathrm{ZnO}$ and $\mathrm{m}-\mathrm{ZrO} 2$ and the bottom most layer for $\mathrm{t}-\mathrm{ZrO} 2$ were held fixed in their bulk positions during all geometry optimizations while the remaining atoms in the slab and in the vacuum layer were allowed to relax. 
(a)
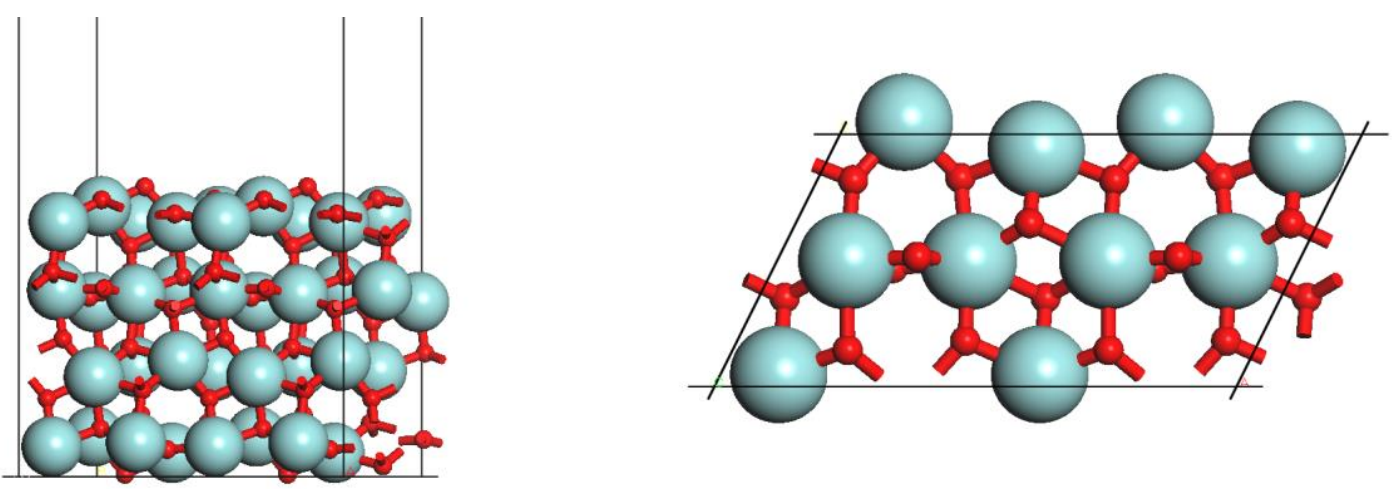

(b)
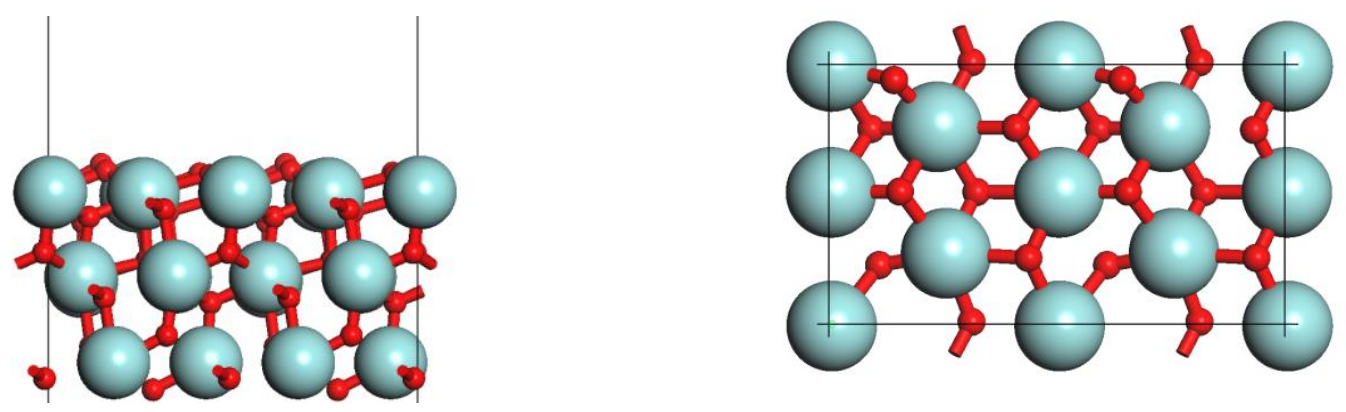

(c)
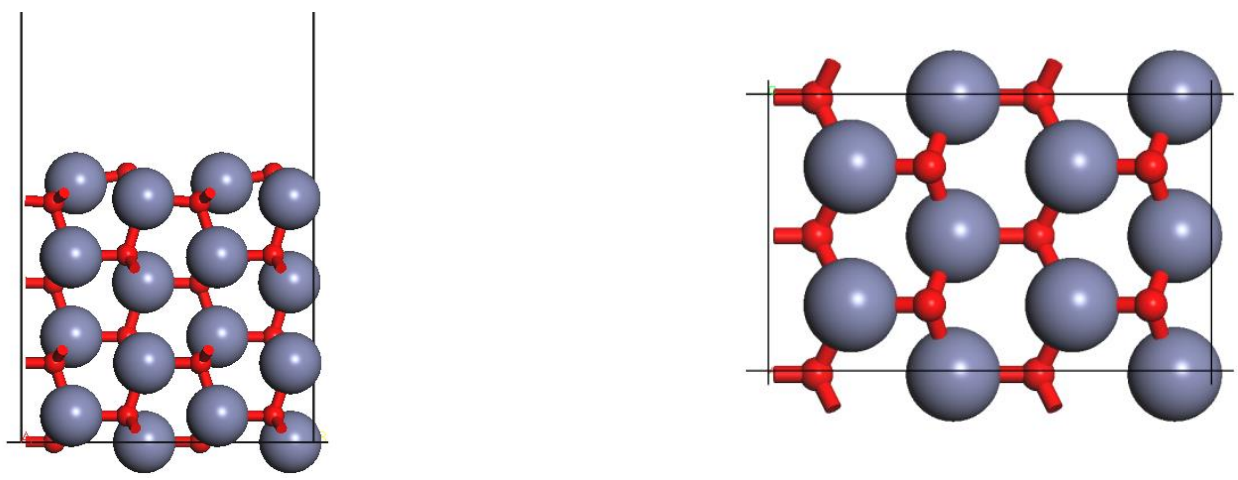

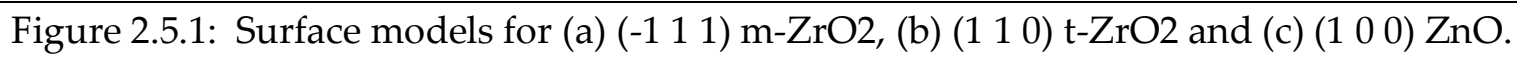

\subsection{Gas Phase Deprotonation Energies of Light Aldehydes and Ketones}

Gas phase properties studied and reported in literature describe effects inherent to the reactants themselves without catalytic influence. Gas phase properties are detailed in the NIST handbook $[2,33-34]$ for $C_{2}-C_{4}$ aldehydes as well as acetone and butanone deprotonation at the methyl position (butanone-1) and deprotonation of the ethyl position (butanone-2) (Table 2.6.1). 
Table 2.6.1: Reported deprotonation energies for $\mathrm{C} 2-\mathrm{C} 4$ aldehydes and $\mathrm{C} 3-\mathrm{C} 4$ ketones in the gas phase [2, 33-34]

\begin{tabular}{lll}
\hline Adsorbate & Deprotonation Energy ${ }^{\mathrm{a}}[\mathrm{kJ} / \mathrm{mol}]$ \\
\hline Ethanal & $1533.1+/-3.4$ \\
Propanal & $1528+/-8.8$ \\
Butanal & $1526+/-8.8$ \\
& & \\
Acetone & 1544 & $+/-8.8$ \\
Butanone-1 & $1540+/-12$ \\
Butanone-2 & $1536+/-12$ \\
\hline
\end{tabular}

The deprotonation reactivity is theorized to be controlled mainly by the stability of the resulting enolate ion [1]. Alkyl groups are weakly donating, so longer carbon chains will provide greater amounts of electron density to the $\alpha$-carbon center and make the final enolate ion less stable. Ketones have two alkyl branches, one on either side of the carbonyl oxygen, which means that ketones will possess even higher amounts of negative charge localized at that $\alpha$-carbon as compared to aldehydes, which only contain one alkyl branch and one neutral acting hydrogen atom on either side of the carbonyl group. As the enolate ion product becomes less stable, the parent enolate formation reaction becomes less favorable and decreases activity for deprotonation for all ketones with respect to aldehydes. At the same time, longer alkyl substrates allow for greater charge delocalization across the entire molecule, which explains why we see deprotonation favorability increase with carbon backbone lengths for both aldehydes and ketones. However, the data above show that this increase in possible delocalization is not overshadowed by the slight increase in electron donation towards the $\alpha$ carbon as even a C4 ketone is still less favorable than a C2 aldehyde for enolate formation. The gas phase literature therefore predicts that an oxygenate's ability to delocalize the resulting negative charge takes precedence over the total reactant size, and is the main determining factor for enolate formation with respect to changing reactant.

\subsection{Adsorption and Oxygen Site Coordination}

The reactant aldehydes or ketones were found to bind to the $\mathrm{Zr}$ as well as the $\mathrm{Zn}$ Lewis acid sites on the surface via electron transfer from their oxygens as well as through a weak 
interaction between the hydrogen at the alpha carbon $\left(\mathrm{C}_{\alpha}\right)$ and a surface oxygen site. A second reactant molecule can adsorb at an adjacent site via the same strong O--M bond and weak O--H interactions. The metal sites that neighbor the bridging oxygen on $\mathrm{m}-\mathrm{ZrO}_{2}$ were chosen as they possess the lowest coordination number. This results in the strongest metal-O binding energy and the highest proton affinity. The $\mathrm{t}-\mathrm{ZrO}_{2}$ and $\mathrm{ZnO}$ surfaces present elevated tri-coordinated oxygen sites that are equally available for enolate formation (Figure 2.5.1).

\subsection{Adsorption of Aldehyde and Ketone Reactants onto Oxide Surfaces}

The adsorption of different aldehydes and ketones onto the $\mathrm{m}-\mathrm{ZrO}_{2}$ surface becomes more favorable with increasing size and degree of substitution of the aldehydes or ketones, with ethanal exhibiting the weakest total heat of adsorption at $-172 \mathrm{~kJ} / \mathrm{mol}$ for two ethanals and the strongest at $-181 \mathrm{~kJ} / \mathrm{mol}$ for two butanals (Table 2.8.1). The $\mathrm{Zr}^{*}--\mathrm{O}$ bond was calculated to be 2.4 $\AA$ for all aldehydes and ketones studied. A secondary interaction between the $\alpha$-carbon's hydrogen and the surface $\mathrm{O}$ is also observed with an $\mathrm{O}^{*}---\mathrm{H}$ bond distance of $1.9 \AA$ for all $\mathrm{Zr}-\mathrm{O}$ sites and surface systems examined. Increasing the carbon chain length results in weaker $\mathrm{C}_{\alpha}-\mathrm{H}$ bond and a greater $\mathrm{H}$ acidity, which is consistent with gas phase deprotonation energies. The more acidic the $\mathrm{C}-\mathrm{H}$ bond, the stronger the $\mathrm{O}^{*}-\mathrm{H}$ interactions between the $\alpha$-carbon's hydrogen and surface oxygen upon oxygenate absorption.

Table 2.8.1: Adsorption energy for two adsorbing molecules, for C2-C4 aldehydes and C3-C4 ketones onto Metal Oxide Surfaces

\begin{tabular}{l|r|r|r}
\hline Adsorbate & $\begin{array}{c}\mathrm{m}-\mathrm{ZrO}_{2} \\
{[\mathrm{~kJ} / \mathrm{mol}]}\end{array}$ & $\begin{array}{c}\mathrm{t}-\mathrm{ZrO}_{2} \\
{[\mathrm{~kJ} / \mathrm{mol}]}\end{array}$ & $\begin{array}{c}\mathrm{ZnO} \\
{[\mathrm{kJ} / \mathrm{mol}]}\end{array}$ \\
\hline Ethanal & -172 & -175 & -170 \\
Propanal & -175 & -177 & -172 \\
Butanal & -181 & -180 & -174 \\
& -181 & -187 & -181 \\
Acetone & -172 & -184 & -176 \\
Butanone & & & \\
\hline
\end{tabular}

The results for the adsorption of different aldehydes on $\mathrm{ZnO}$ and $\mathrm{ZrO}_{2}$ reported in Table 2.8.1 shows that increasing the chain length increases the $\mathrm{C}_{\alpha}-\mathrm{H}$ acidity along with the aldehyde 
adsorption energies. The changes in the $\alpha$-carbon acidity between aldehydes appear to be correlated with the changes in adsorption energy differences. The changes in the adsorption energies, however, vary by only a few $\mathrm{kJ} / \mathrm{mol}$. As a result, it is difficult to distinguish catalytic surface effects on adsorption based on energetic values alone.

\subsection{Intrinsic Energies for Enolate Formation Reaction on Oxide Surfaces}

Aldol condensation is thought to proceed via the activation of the $\mathrm{C} \alpha-\mathrm{H}$ bond at the Lewis acid $(\mathrm{M})$ and base $\left(\mathrm{O}^{*}\right)$ site pair to form an enolate that binds to the metal center via its oxygen and a proton that is coordinated to the basic $\mathrm{O}^{*}$ site. The reaction proceeds via $\mathrm{C} \alpha-\mathrm{H}$ activation and transfer of the acidic $\mathrm{H}$ from the $\mathrm{C} \alpha$ to $\mathrm{O}^{*}$ site on the surface as is shown in Figure 2.9.1.

\section{Propanal Adsorption $\quad$ Transition State Enolate Formation}

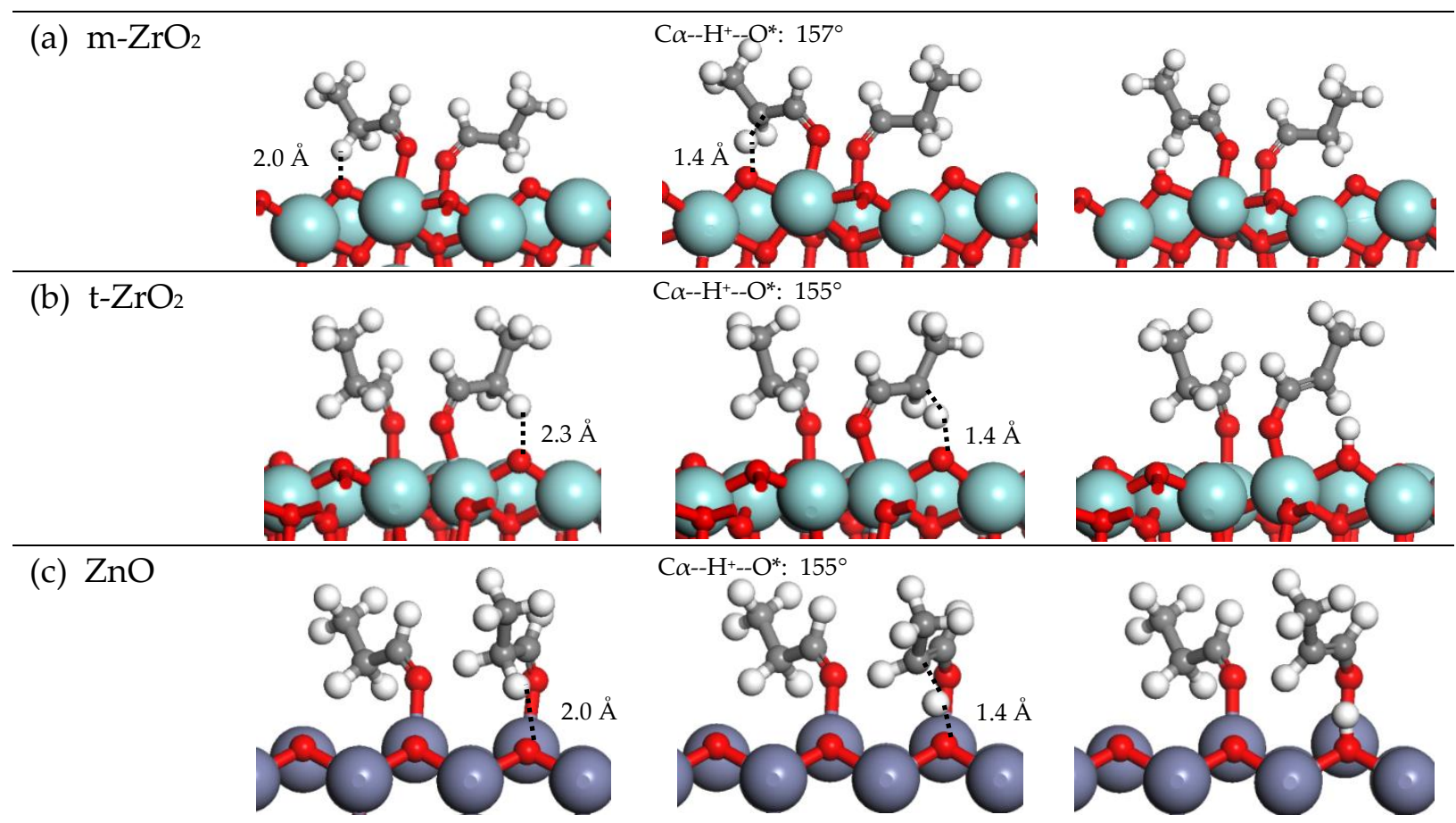

Figure 2.9.1: Surface models of aldehyde adsorption, transition state, and enolate intermediate for propanal enolate formation over (a) $\mathrm{m}-\mathrm{ZrO}_{2}$, (b) $\mathrm{t}-\mathrm{ZrO}_{2}$, and (c) $\mathrm{ZnO}$ surfaces.

Measurements of the $\mathrm{C} \alpha-\mathrm{H}--\mathrm{O}^{*}$ bond length and the $\mathrm{C} \alpha--\mathrm{H}(+)--\mathrm{O}^{*}$ angle at the aldehyde states and transition states show that the propanal leans over during deprotonation in order to 
place the proton in close proximity of the $\mathrm{O}^{*}$ site at the transition state, with similar bond lengths and angles observed for all $\mathrm{ZrO} 2$ and $\mathrm{ZnO}$ surfaces. A more detailed analysis of the charge presented later in this section shows the heterolytic activation of the $\mathrm{C} \alpha-\mathrm{H}$ bond to form a proton and an enolate with the negative charge delocalized over the surface $\mathrm{CH} 3 \mathrm{CHCH}=\mathrm{O}$ enolate.

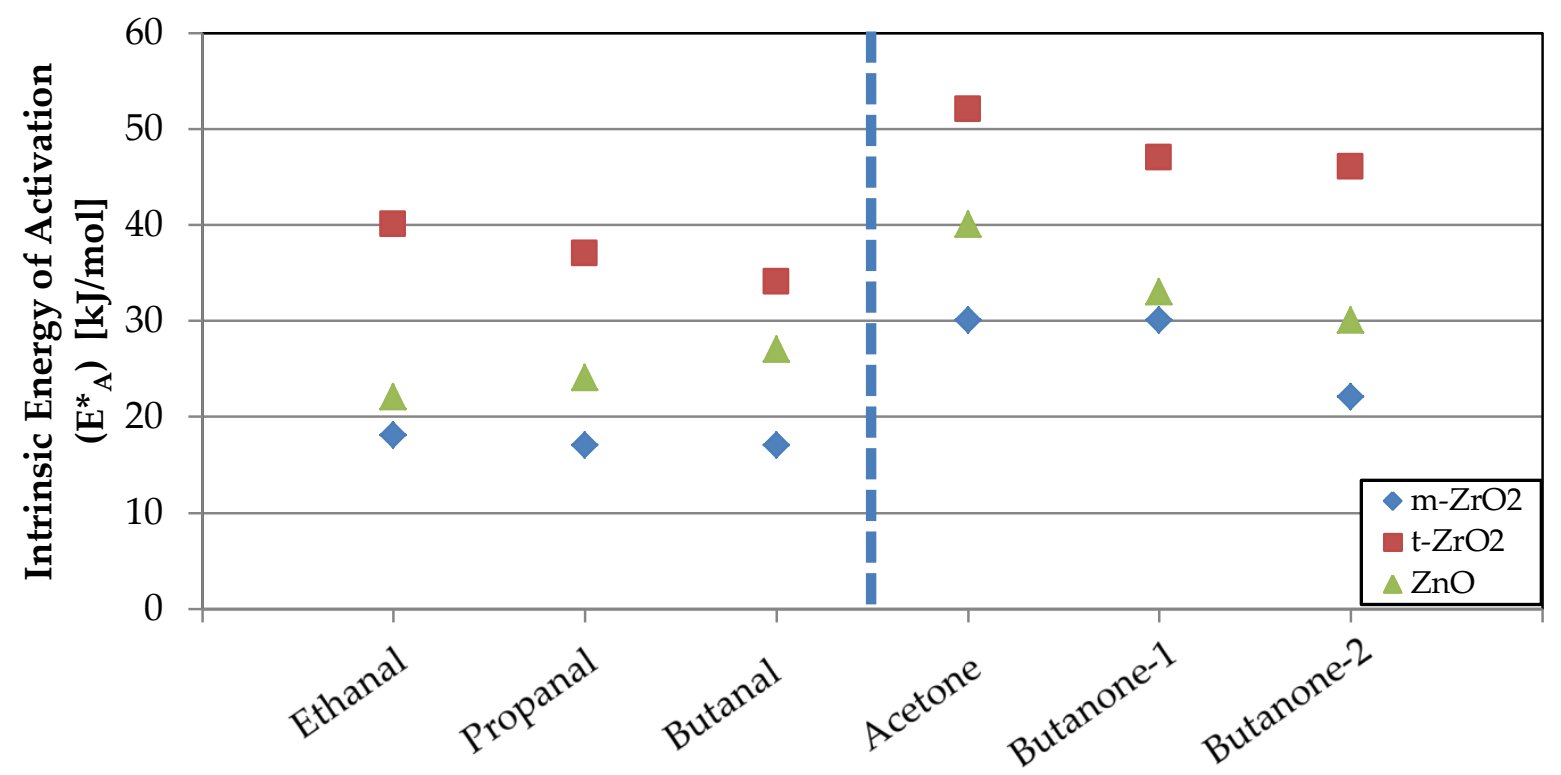

Figure 2.9.2: Intrinsic energy of activation of $\mathrm{C}_{2}-\mathrm{C}_{4}$ aldehydes and $\mathrm{C}_{3}-\mathrm{C}_{4}$ ketones over $\mathrm{m}-\mathrm{ZrO}_{2}$, $\mathrm{t}-\mathrm{ZrO}_{2}$, and $\mathrm{ZnO}$.

We examined a range of $\mathrm{C}_{2}-\mathrm{C}_{4}$ aldehydes and ketones which include ethanal, propanal, butanal, acetone, and both butanone instances where the $\mathrm{C}_{\alpha}-\mathrm{H}$ bond is cleaved on the methyl side (butanone-1) or the ethyl side (butanone-2). The calculated activation barriers for enolate formation for all of the aldehydes examined were calculated to be low over m- $\mathrm{ZrO}_{2}$, with propanal and butanal both demonstrating the lowest energy barriers of $17 \mathrm{~kJ} / \mathrm{mol}$ (Figure 2.9.2). The barriers for the ketones shown in Figure 2.9.2 are notably higher with acetone exhibiting an energy of $30 \mathrm{~kJ} / \mathrm{mol}$ and butanone resulting in barriers of 30 and $22 \mathrm{~kJ} / \mathrm{mol}$ for the deprotonation of methyl and ethyl side chains, respectively. The increased activity for aldehyde enolate formation is consistent with gas phase deprotonation energies reported in the literature [14], thus emphasizing the importance of the resulting enolate ion's stability inherent to the reactant 
molecule. When comparing intrinsic values across the oxide surfaces, the differences in calculated intrinsic energies reveal that the surface further stabilizes each reactant in a different manner.

The Bader charge distribution of reactions on $\mathrm{m}-\mathrm{ZrO}_{2}$ illustrates that, as the $\mathrm{H}+$ transfers from the carbon chain to the oxide surface, both the enolate and reactive proton experience significant changes in charge, with the enolate carbon gaining in negative charge from -0.26 at the reactant state to -0.74 at the product state, indicating the formation of a charged enolate intermediate (Figure 2.9.3). Similarly, the transferring $\mathrm{H}^{+}$shows a positive gain in charge from +0.21 to +0.58 , which is characteristic of a proton transfer event. Conversely, during this proton transfer event, the second adsorbed reactant experiences negligibly small charge transfer of +0.01 , suggesting that this adsorbate acts as a spectator during the enolate formation reaction. The oxide surface experiences minimal charge transfer with a total change of only +0.09 charge, consistent with literature regarding charge neutrality of zirconia surfaces [16].

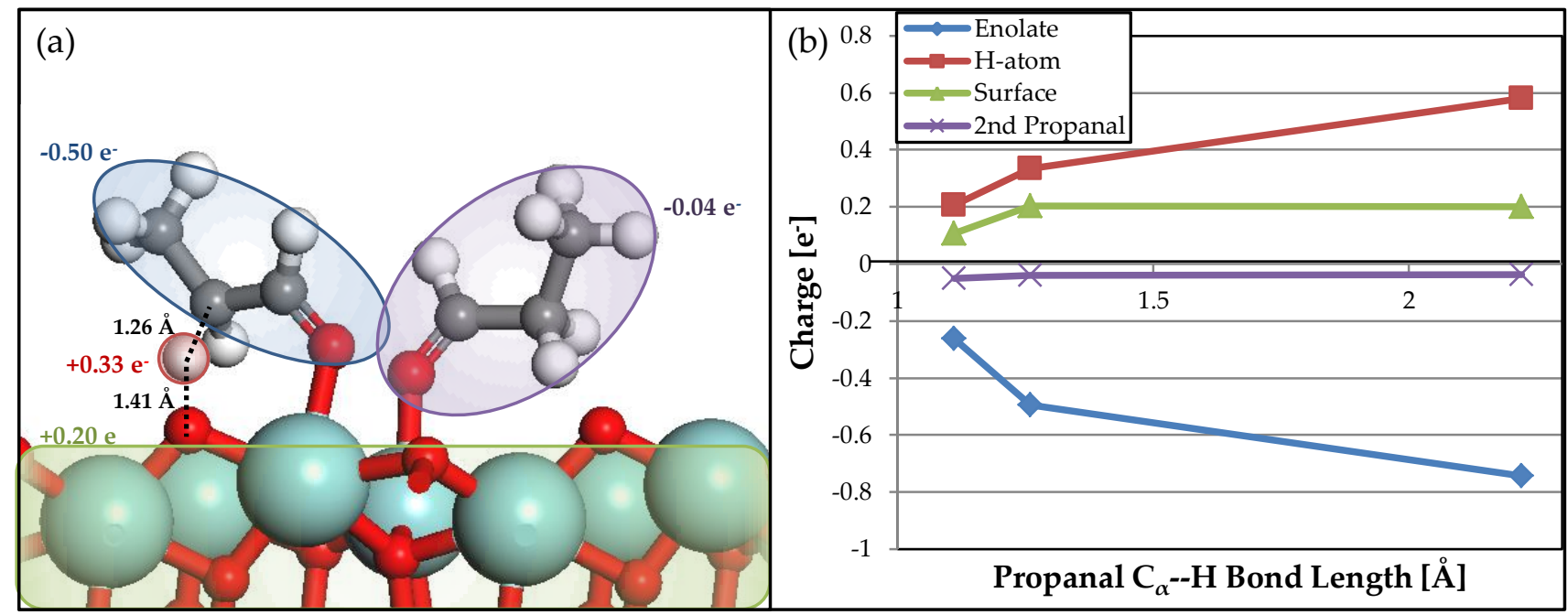

Figure 2.9.3: (a) The structure of the transition state for the activation of propanal at the $\mathrm{Zr}-\mathrm{O}$ site pair to form the enolate on $\mathrm{m}-\mathrm{ZrO}_{2}$. (b) The changes in charge on the $\mathrm{H}$ (red), enolate ion (blue), and $\mathrm{ZrO}_{2}$ surface (green) and the co-adsorbed propanal (purple) that occur as function of the $\mathrm{C}_{\alpha}-\mathrm{H}$ bond length along the reaction coordinate involved in the formation of the enolate. 
Collectively, these results suggest that the enolate forms via a proton transfer with minimal involvement of nearby adsorbate molecules. If there is any charge transfer from the m$\mathrm{ZrO}_{2}$ surface to the adsorbed intermediates, it takes place either during adsorption or during elementary steps following enolate formation. The proton and the enolate that form at the $\mathrm{Zr}$ and $\mathrm{O}$ sites remain in close proximity to one another upon enolate formation, with a distance of $1.9 \AA$ between the surface proton and $C_{\alpha}$ of the enolate. The close proximity allows for further enolate stabilization through intermolecular coulombic interactions between the adsorbed surface proton and enolate intermediate that would not be present in gas phase reactions.

A comparison of the Bader charges that occur on the $\mathrm{H}$ atom, the enolate, the $\mathrm{ZrO}_{2}$ surface and co-adsorbed alkanal for the reactions of ethanal and acetone over $\mathrm{m}-\mathrm{ZrO}_{2}$ reported in Figure 2.9.4 provide insights into the differences in the activation energies between the aldehydes and ketones. The results reveal that, even though the enolates for ethanal and acetone have the same accumulation of negative charge at -0.6 , the $C_{\alpha}$ center on ethanal is less charged (-0.18) in the transition state than acetone (-0.21). This difference is predominantly due to differences in delocalization potential of aldehydes and ketones, in which the primary $\mathrm{C}_{\alpha}$ of acetone cannot delocalize the negative charge as well as the primary $\mathrm{C}_{\alpha}$ center of ethanal.

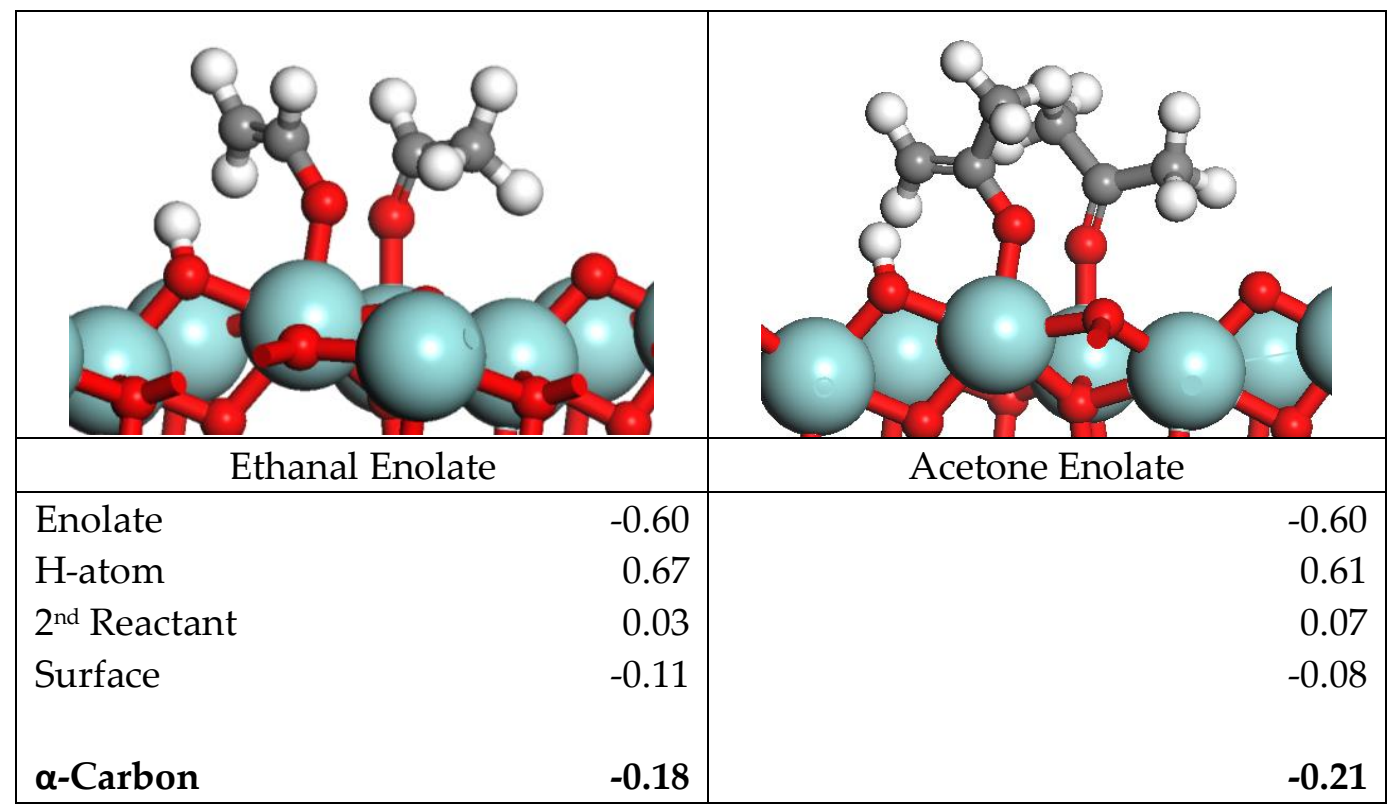

Figure 2.9.4: Bader charge analysis of ethanal vs acetone over $\mathrm{m}-\mathrm{ZrO}_{2}$. 
While the actual intrinsic activation energies for enolate formation are higher on $\mathrm{t}-\mathrm{ZrO}_{2}$ versus those on $\mathrm{m}-\mathrm{ZrO}_{2}$, the relative changes in the intrinsic barriers for different aldehyde and ketone over $\mathrm{t}-\mathrm{ZrO}{ }_{2}$ are similar to those calculated for $\mathrm{m}-\mathrm{ZrO}$. As in $\mathrm{m}-\mathrm{ZrO}$ and gas phase enolate formation, butanal results in the lowest intrinsic barrier of $27 \mathrm{~kJ} / \mathrm{mol}$ over $\mathrm{t}-\mathrm{ZrO}_{2}$. Similarly, the intrinsic barriers for the ketones over $\mathrm{t}-\mathrm{ZrO}_{2}$ are significantly higher than those of the aldehydes, with acetone activation retaining the highest energetic barrier $52 \mathrm{~kJ} / \mathrm{mol}$. The, $\mathrm{ZnO}$ systems, on the other hand, differ significantly from both the $\mathrm{m}-\mathrm{ZrO}_{2}$ and the $\mathrm{t}-\mathrm{ZrO}_{2}$ surfaces, as the barriers for enolate formation from the linear aldehydes becomes more difficult with increasing molecular size. This trend is counter to the expected increase in enolate formation reactivity with increased acidity, and therefore suggests that the surface preferentially aids in stabilizing smaller enolate intermediates.

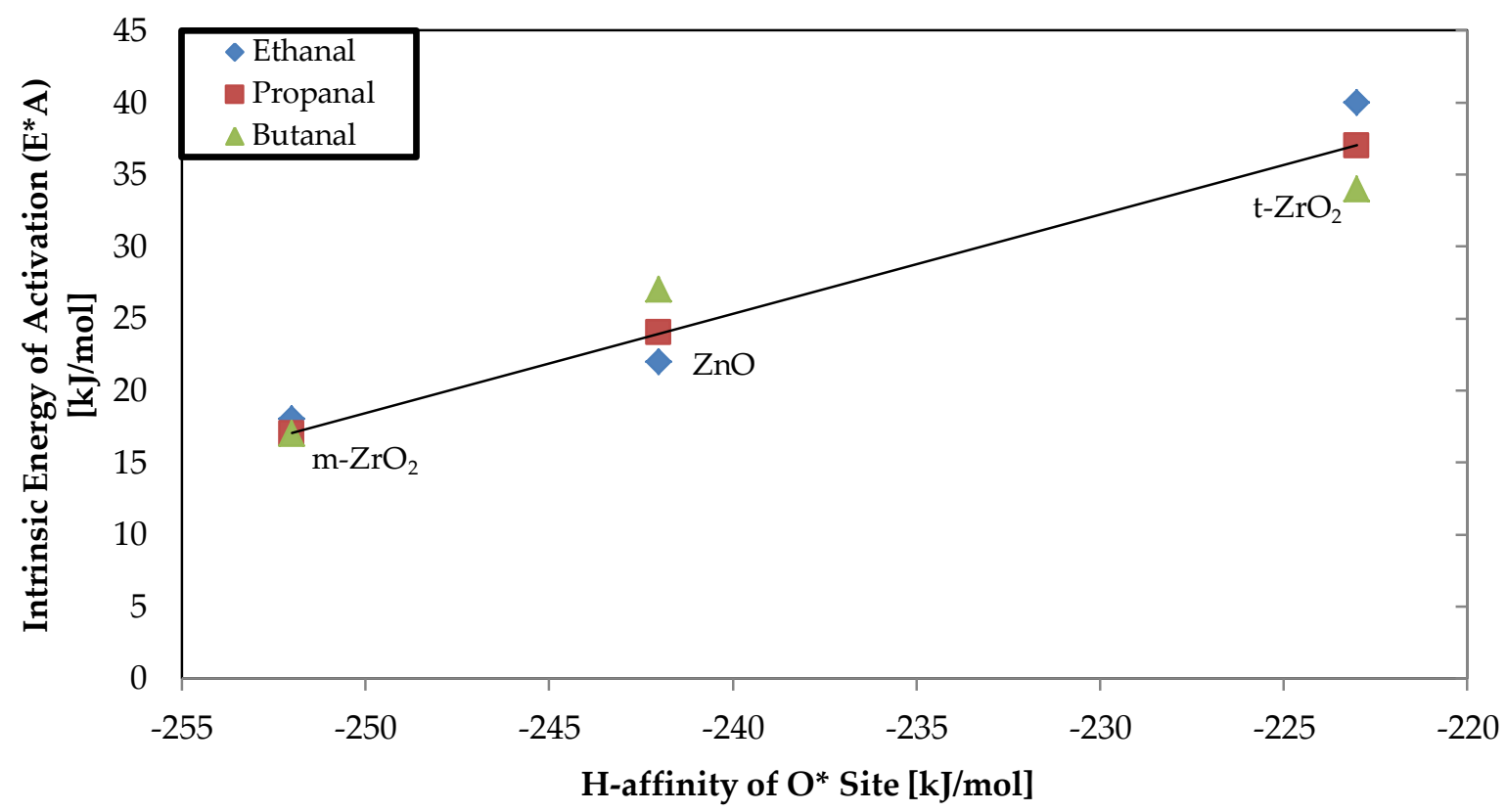

Figure 2.9.5: Intrinsic activation energies for the $\mathrm{C}_{2}-\mathrm{C}_{4}$ aldehydes to react to form the corresponding enolate vs $\mathrm{H}$-affinity for the $\mathrm{m}-\mathrm{ZrO}_{2}, \mathrm{ZnO}$, and $\mathrm{t}-\mathrm{ZrO}_{2}$ surfaces.

The intrinsic activation barriers for enolate formation were plotted as a function of surface oxygen site H-affinities to help elucidate the importance of the interaction of the proton that forms upon deprotonation and the oxide surface. The results in Figure 2.9.5 show a direct 
correlation between the surface basicity and enolate formation energy. The H-affinities reported in Figure 2.9.5 suggest that the relative basicities of the surface decrease from $\mathrm{m}-\mathrm{ZrO}_{2}>$ $\mathrm{ZnO}>\mathrm{t}-\mathrm{ZrO}_{2}$. The $\mathrm{H}$-affinity, which is defined as the energy associated with adding an $\mathrm{H}$ atom to a bare catalyst surface, provides an approximate measure of the surface oxygen's basicity. The relative differences in the basicity between $\mathrm{m}-\mathrm{ZrO}$ and $\mathrm{t}-\mathrm{ZrO}_{2}$ is likely due to differences in oxygen coordination number, where the $\mathrm{m}-\mathrm{ZrO}_{2}$ involve 2-coordinate bridge oxygen sites for proton acceptance which bind the $\mathrm{H}$ more strongly than the 3-coordinate oxygen sites on the $\mathrm{t}$ $\mathrm{ZrO}_{2}$ surface.

Of all oxide surfaces within this study, $\mathrm{m}-\mathrm{ZrO}_{2}$ consistently yielded the lowest intrinsic activation energies for all of the small oxygenates examined. The intrinsic activation barrier for propanal enolate formation for $\mathrm{m}-\mathrm{ZrO}_{2}$ is $17 \mathrm{~kJ} / \mathrm{mol}$ whereas $\mathrm{ZnO}$ and $\mathrm{t}-\mathrm{ZrO}{ }_{2}$ values for propanal enolate formation are higher at $24 \mathrm{~kJ} / \mathrm{mol}$ and $37 \mathrm{~kJ} / \mathrm{mol}$, respectively. Considering the slight differences in intrinsic energy due to molecular size of $2-4 \mathrm{~kJ} / \mathrm{mol}$, the data illustrated in Figure 2.8.5 suggests that catalyst basicity has a greater impact on the intrinsic energies than variations that result from changes in carbon chain length. Increases in $\mathrm{H}$-affinity of the $\mathrm{O}^{*}$ site from $-252 \mathrm{~kJ} / \mathrm{mol}$ for $\mathrm{m}-\mathrm{ZrO}_{2}$ to $-223 \mathrm{~kJ} / \mathrm{mol}$ for $\mathrm{t}-\mathrm{ZrO}_{2}$ yield a $20 \mathrm{~kJ} / \mathrm{mol}$ decrease in intrinsic energy barrier for propanal, whereas increasing the carbon chain length from ethanal to butanal over $\mathrm{t}-\mathrm{ZrO}_{2}$ results in a $4 \mathrm{~kJ} / \mathrm{mol}$ decrease in intrinsic energy. However, differences between ketones and aldehydes may be equal in importance to catalyst oxygen site basicity, with differences in intrinsic activation energy of $12 \mathrm{~kJ} / \mathrm{mol}$ between propanal and acetone over m$\mathrm{ZrO}_{2}$.

\subsection{Apparent Activation Energies and Gibbs Free Activation Energies for Enolate Formation}

The intrinsic activation barriers were used along with the heats of adsorption to assess and calculate the apparent barriers. The calculated entropy differences associated with adsorption and forming the enolate transition state allowed us to subsequently calculate the relative free energies associated with the reaction. The calculated apparent free energies of activation to form the enolate are plotted for all of the different $\mathrm{C}_{2}-\mathrm{C}_{4}$ aldehydes and ketones considered in Figure 2.10.1. The results show that all of the enolate formation energies have 
moderately positive Gibbs free energies of activation when referenced to the gas phase of all reactants. The strong interaction between the oxygen of the carbonyl of the reactant and the

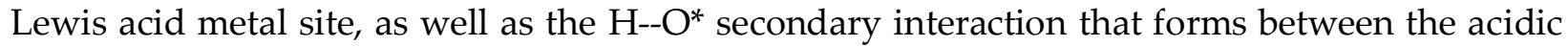
$\mathrm{C}_{\alpha}-\mathrm{H}$ and the basic $\mathrm{O}^{*}$ surface atom, result in a significant loss in the degrees of freedom of the reactant, and a large loss in entropy. This loss of entropy results in fairly high positive free energies of adsorption for both of the alkanal reactants onto the oxide surfaces. ।

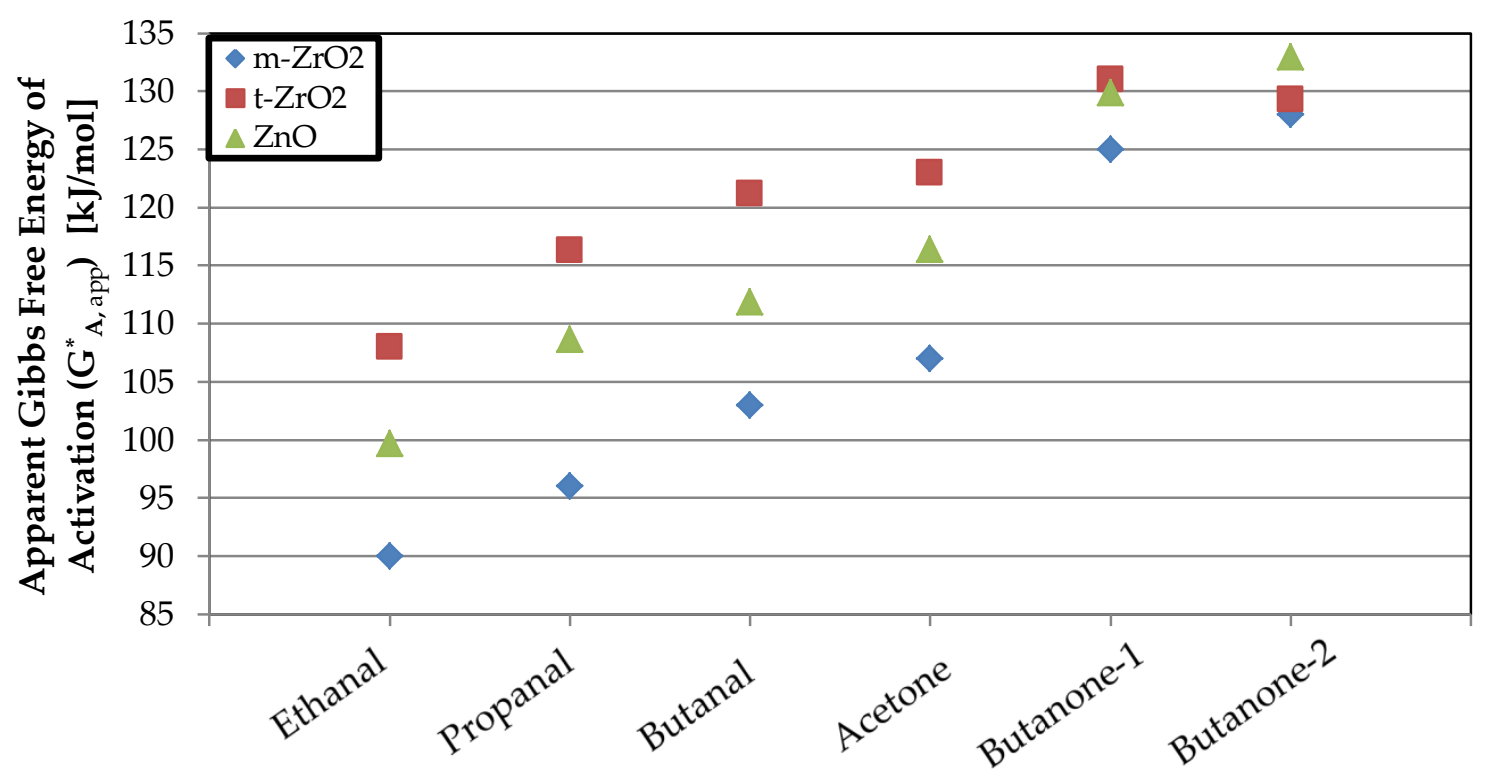

Figure 2.10.1: Apparent Gibbs free energy of activation taken with respect to the gas phase for $\mathrm{C}_{2}-\mathrm{C}_{4}$ aldehydes and $\mathrm{C}_{3}-\mathrm{C}_{4}$ ketones at $523 \mathrm{~K}$ over (a) $\mathrm{m}-\mathrm{ZrO}_{2}$, (b) $\mathrm{t}-\mathrm{ZrO}_{2}$, and (c) $\mathrm{ZnO}$.

The free energies of activation of the different alkanals shown in Figure 2.10.1 systematically increase with increasing chain length from ethanol $<$ propanal $<$ butanal, as well as acetone $<$ butanone- $1<$ butanone- 2 as well as increasing the degree of substitution for the reactions on oxide surfaces examined, except in the case of $\mathrm{t}-\mathrm{ZrO}_{2}$ for butanone-2 in which $\mathrm{t}$ $\mathrm{ZrO}_{2}$ yields a lower apparent free energy than $\mathrm{ZnO}$. The lowest free energy barriers were those for ethanal with energies of $90 \mathrm{~kJ} / \mathrm{mol}, 100 \mathrm{~kJ} / \mathrm{mol}$ and $108 \mathrm{~kJ} / \mathrm{mol}$ for the $\mathrm{m}-\mathrm{ZrO}$, $\mathrm{ZnO}$ and t$\mathrm{ZrO}_{2}$ surfaces, respectively. The apparent free energy barriers for the formation of enolate species on $\mathrm{m}-\mathrm{ZrO}_{2}$ ranges increase from $90 \mathrm{~kJ} / \mathrm{mol}$ for ethanal to $128 \mathrm{~kJ} / \mathrm{mol}$ for 2-butanone. An 
analysis of free energy barriers across oxide surfaces indicates that the $\mathrm{m}-\mathrm{ZrO}_{2}$ surface is the most active, followed by $\mathrm{ZnO}$, and with $\mathrm{t}-\mathrm{ZrO}_{2}$ as the least active for enolate formation.

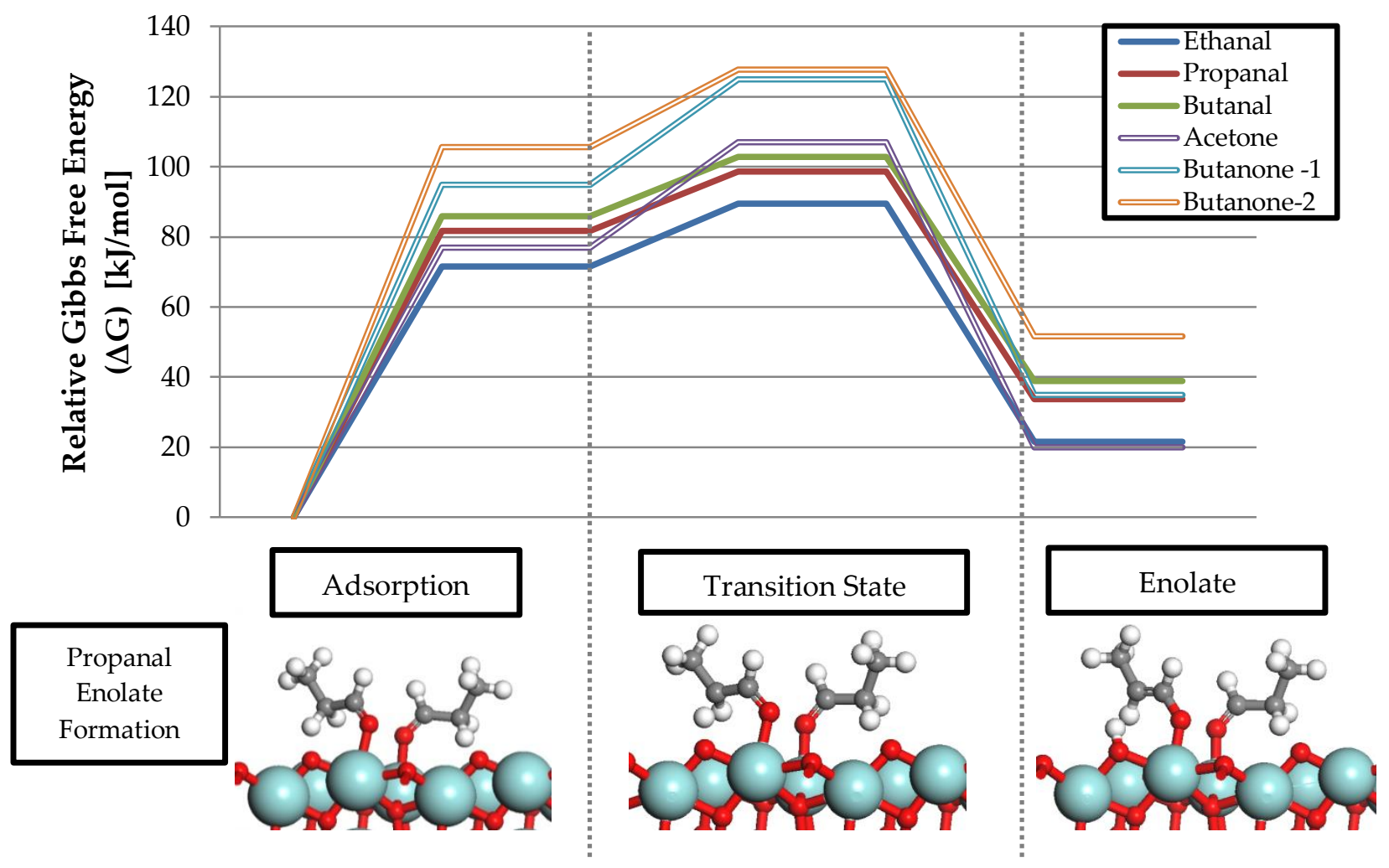

Figure 2.10.2: Gibbs free energy diagram at $523 \mathrm{~K}$ for enolate formation of $\mathrm{C}_{2}-\mathrm{C}_{4}$ aldehydes and C3-C4 ketones over $\mathrm{m}-\mathrm{ZrO}_{2}$.

\subsection{Born-Haber Cycle Analysis and Effect of Catalyst-Intermediate Interaction}

In order to elucidate the importance of the elementary thermochemical bond making and breaking steps in controlling the activation of the alkanal we use a Born-Haber cycle analysis to analyze the change in energy from the adsorbed alkanal reactant state to the enolate$-\mathrm{H}---\mathrm{O}^{*}$ transition state. The apparent activation energy for enolate formation can be broken down into a sequence of simplified thermochemical steps that follow the elementary gas phase reactions, and then sequentially adds each of these intermediates back onto the surface to form the transition state (Figure 2.11.1) [35]. This process separates the apparent activation energy for enolate formation into 3 steps: the deprotonation energy (DPE) of the gas phase alkanal to 
form the $\mathrm{RC}(-) \mathrm{H}-\mathrm{CH}=\mathrm{O}$ enolate and a free proton $\left(\mathrm{H}^{+}\right)$, the adsorption of the $\mathrm{H}^{+}$to the $\mathrm{O}$ on the oxide surface, and the adsorption of the enolate $\mathrm{RC}\left({ }^{(-)} \mathrm{H}-\mathrm{CH}=\mathrm{O}\right.$ enolate at the neighboring metal site on the protonated oxide surface in the transition state. The binding of the enolate is comprised of the enolate adsorption energy along with the coulombic interaction energy between the negatively charged surface enolate and positively charged adsorbed proton. Once isolated, the coulombic interaction can be calculated directly using the enthalpic energies of activation and then compared against the Gibbs free energies of activation to better understand the effect of transition state stabilization on the observed activity for enolate formation (Figure 2.11.2).

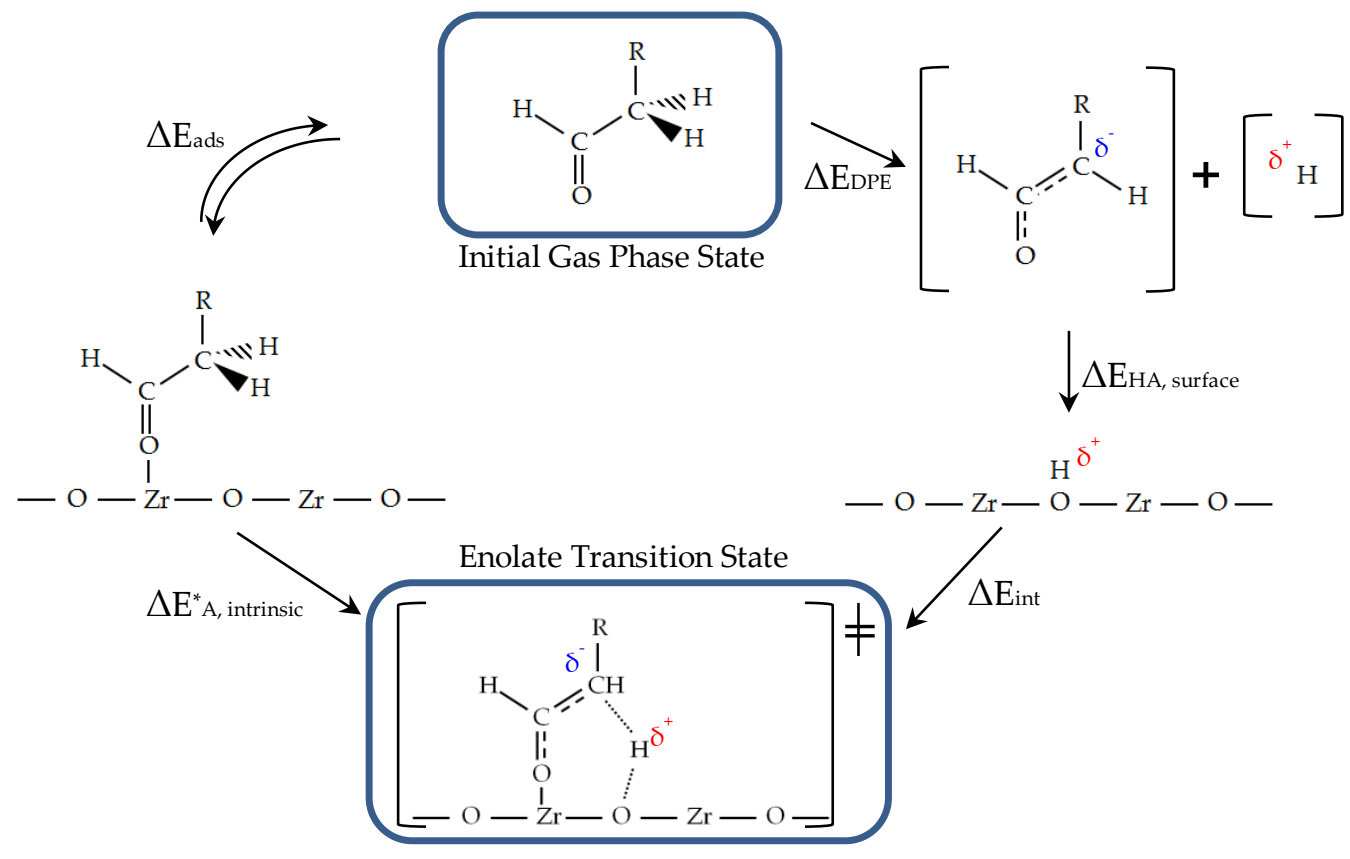

Figure 2.11.1: Born-Haber cycle diagram for enolate formation of aldehydes taken with respect to the gas phase. Left side energies consist of aldehyde adsorption energy ( $\Delta$ Eads) and intrinsic activation energy for enolate formation $\left(\Delta \mathrm{E}^{*} \mathrm{~A}\right.$,intrinsic). Right side energies consist of deprotonation energy of gas phase molecule $(\triangle \mathrm{EDPE}), \mathrm{H}$-affinity of the oxide surface $(\Delta \mathrm{EHA}$, surface), and enolate adsorption energy and surface intermediate coulombic interactions ( $\Delta$ Eint). 
The deprotonation energies provide important insight into how changes in the reactant molecules can influence the activation energies by stabilizing the conjugate base that forms, thus resulting in lower deprotonation energies whereas the changes in the proton affinities of the oxide surface provide insights into the basicity and reactivity of the surface. We found herein that the coulombic interactions between the enolate and the protonated oxide surface appears to reliably capture both the changes in the reactant and the surface in stabilizing the transition state as the coulombic interactions correlated linearly with the apparent Gibbs free energy of activation to form the enolate for all of the alkanal systems examined as shown in Figure 2.11.2.

The calculated coulomb energy here involves the interaction between the negative enolate and positive surface proton. The smaller aldehydes result in stronger coulombic stabilization as they act as a hard base with greater localized negative charge that can better stabilize the neighboring proton. Of the aldehydes tested, ethanal consistently possessed the greatest coulombic interaction with $-202 \mathrm{~kJ} / \mathrm{mol},-146 \mathrm{~kJ} / \mathrm{mol}$, and $-142 \mathrm{~kJ} / \mathrm{mol}$ for $\mathrm{m}-\mathrm{ZrO}_{2}, \mathrm{ZnO}$, and $\mathrm{t}-\mathrm{ZrO}_{2}$ systems, respectively. It is also noted that the calculated differences between ethanal and propanal are significantly larger than the coulombic interaction energy differences between propanal and butanal on the different oxide surfaces as is shown along the x-axis in Figure 2.11.2. The larger difference between $C_{2}$ and $C_{3}$ aldehydes is due to the difference in degree of substitution at the $\alpha$-carbon, as the $\alpha$-carbon on ethanal is a primary carbon that is not able to delocalize the negative charge as effectively as the secondary $\alpha$-carbon in propanal, and as such acts as a harder base.

A similar correlation between activation energies and coulombic interaction energies also exists for ketone systems. As observed for aldehydes, short carbon chains possess greater affinities for the charged surface with acetone possessing the greatest interaction energy of -199 $\mathrm{kJ} / \mathrm{mol},-144 \mathrm{~kJ} / \mathrm{mol}$, and $-139 \mathrm{~kJ} / \mathrm{mol}$ for $\mathrm{m}-\mathrm{ZrO}_{2}, \mathrm{ZnO}$, and $\mathrm{t}-\mathrm{ZrO}_{2}$ systems, respectively. The difference in calculated interaction energy between acetone and 1-butanone is also larger than the difference in interaction energy between 1-butanone and 2-butanone. This indicates that the enolate stabilization as result of the longer carbon backbone of 1-butanone over acetone outweighs the delocalization differences that result from the increased substitution at the 
primary $\alpha$-carbon position in 1-butanone over the secondary 2-butanone for the respective enolates.
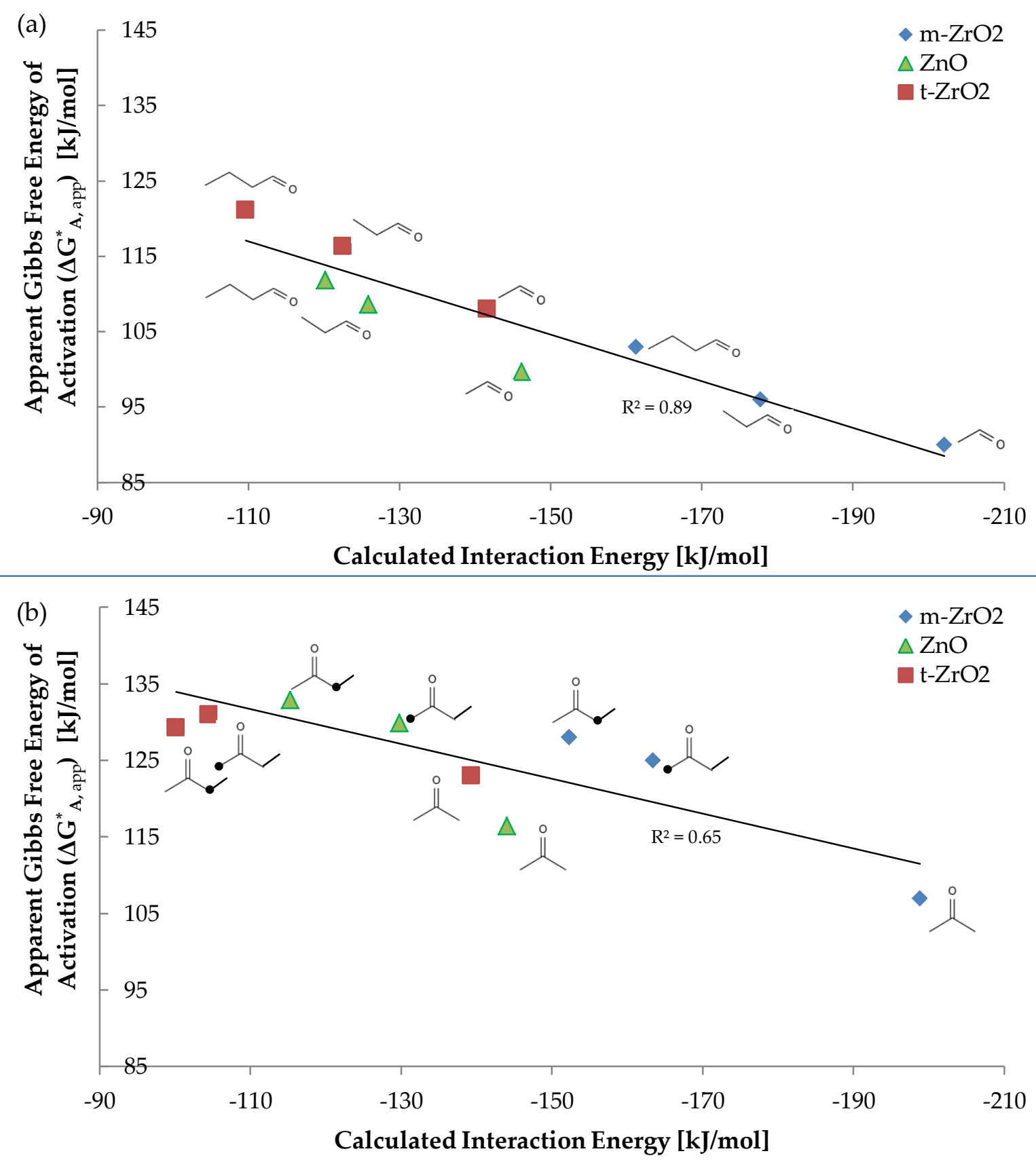

Figure 2.11.2: Linear relationship between apparent Gibbs free energy of activation and calculated enolate-proton interaction energy for (a) $\mathrm{C}_{2}-\mathrm{C}_{4}$ aldehydes and (b) $\mathrm{C}_{3}-\mathrm{C}_{4}$ ketones. 
The analysis of coulombic interactions and activation energies reveals several important trends. The first is that the interaction energy tends to be dominated by the choice of the oxide surface, indicating that the surface interaction with the charged intermediate is likely controlled by the acid and base properties of the oxide surface. Second, increased stabilization of the charged intermediate is directly translated into lowering of free energy barriers. Consequently, the oxide surfaces appear to increase reaction rates through a stabilization of the charged intermediate.

The intrinsic heats of reaction for enolate formation are fairly exothermic for all of the reactants and surfaces tested, at roughly $-50 \mathrm{~kJ} / \mathrm{mol}$ for aldehydes and $-60 \mathrm{~kJ} / \mathrm{mol}$ for ketones on both $\mathrm{m}-\mathrm{ZrO}_{2}$ and $\mathrm{t}-\mathrm{ZrO}_{2}$. The exothermicity for enolate formation likely derives from the strong interaction between the negatively charged enolate with the adsorbed proton as well as the catalyst surface as compared to the weaker interaction of the adsorbed aldehyde in the reactant state. The large exothermicity of the reaction together with the intrinsic activation barrier for the forward reaction $(10-40 \mathrm{~kJ} / \mathrm{mol})$ results in a high barrier for the reverse reaction, which range from $70 \mathrm{~kJ} / \mathrm{mol}$ to $90 \mathrm{~kJ} / \mathrm{mol}$ across all of the reactants examined on the $\mathrm{m}-\mathrm{ZrO}{ }_{2}$ surface. These results indicate the high difficulty for reforming the reactant and the likelihood of irreversibility for enolate formation.

\subsection{C-C Bond Formation Step in Aldol Condensation}

The negatively charged enolate that forms can subsequently carry out a nucleophilic attack of the carbon center of the carbonyl on a neighboring alkanal to form a C-C bond. As the enolate formation step was analyzed with respect to the co-adsorption of both alkanals onto the metal-oxide surface, the intrinsic activation barriers for C-C coupling were calculated with respect to the co-adsorbed alkanal and the enolate for both self-coupling and cross-coupling reactions in order to gain insight about the possible product distributions for mixed reactant pools.

The DFT-calculated intrinsic activation energies for the self- and cross-coupling pairs for $\mathrm{C}_{2}-\mathrm{C}_{4}$ aldehydes over $\mathrm{m}-\mathrm{ZrO}_{2}$ are compared against one another in Figure 2.12.1. The results show a clear dependence of the barrier on the size and degree of substitution of the reactant 
molecule. The lowest energy barriers are associated with the coupling of smallest aldehyde molecules. The activation barriers increase with increasing molecular size and substitution. The self-coupling of ethanal, for example, occurs with an intrinsic activation energy of $6 \mathrm{~kJ} / \mathrm{mol}$, while that of butanal proceeds with a barrier of $31 \mathrm{~kJ} / \mathrm{mol}$. This size dependence of activation energies is likely due to the steric penalties incurred when the neighboring carbon tails or substituents are forced into close proximity during the C-C coupling reaction. This increase in intermolecular repulsive interactions between larger adsorbates results in a net increase of the activation energy.

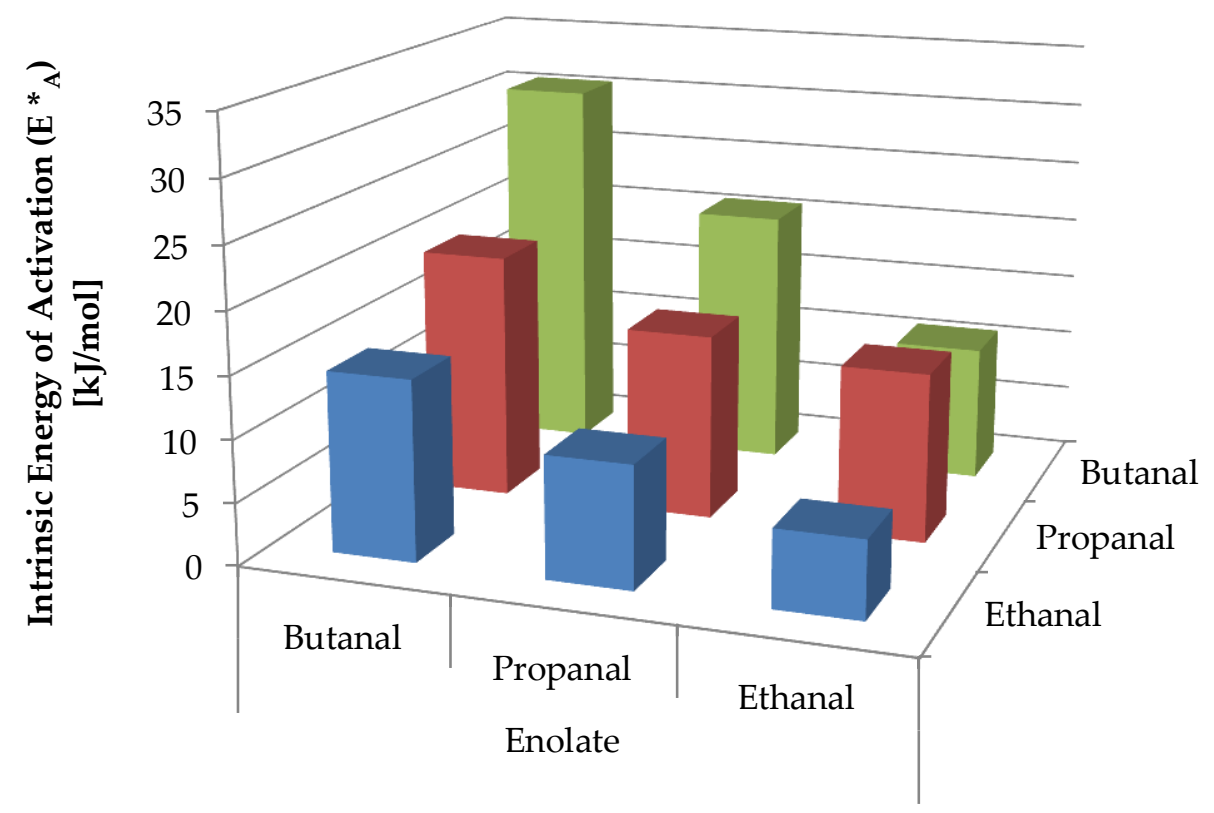

Figure 2.12.1: Intrinsic activation energies for $C-C$ bond formation of $\mathrm{C}_{2}-\mathrm{C}_{4}$ aldehydes over m$\mathrm{ZrO}_{2}$.

Comparison of the self-coupling of propanal the cross-coupling of propanal and acetone reveals the significant role of functional group on C-C bond formation (Figure 2.12.2). The selfcoupling of acetone was not considered as the apparent free energy of activation to form the enolate was $29 \mathrm{~kJ} / \mathrm{mol}$ higher than that of propanal, and as such would result in much lower surface concentrations than that for the propanal enolate. The intrinsic barriers for propanal self-coupling was calculated to be $15 \mathrm{~kJ} / \mathrm{mol}$ while the barrier for propanal/acetone cross- 
coupling was higher at $44 \mathrm{~kJ} / \mathrm{mol}$. The observed differences between propanal self-coupling and propanal-acetone cross-coupling likely stems from a balance between the repulsive steric interactions and the attractive coulombic interactions between the two carbon centers that ultimately go on to form the $\mathrm{C}-\mathrm{C}$ bond. The differences in steric repulsion are due to the differences in the coordination of the two carbon centers. The self-coupling of two aldehydes involves two secondary carbon centers whereas the coupling of propanal and acetone involves the coordination of a secondary carbon bonding to a tertiary carbon center. The tertiary carbon center results in greater steric repulsion, thus resulting in an increase in the activation barrier over that involving the coupling of two secondary carbon centers.

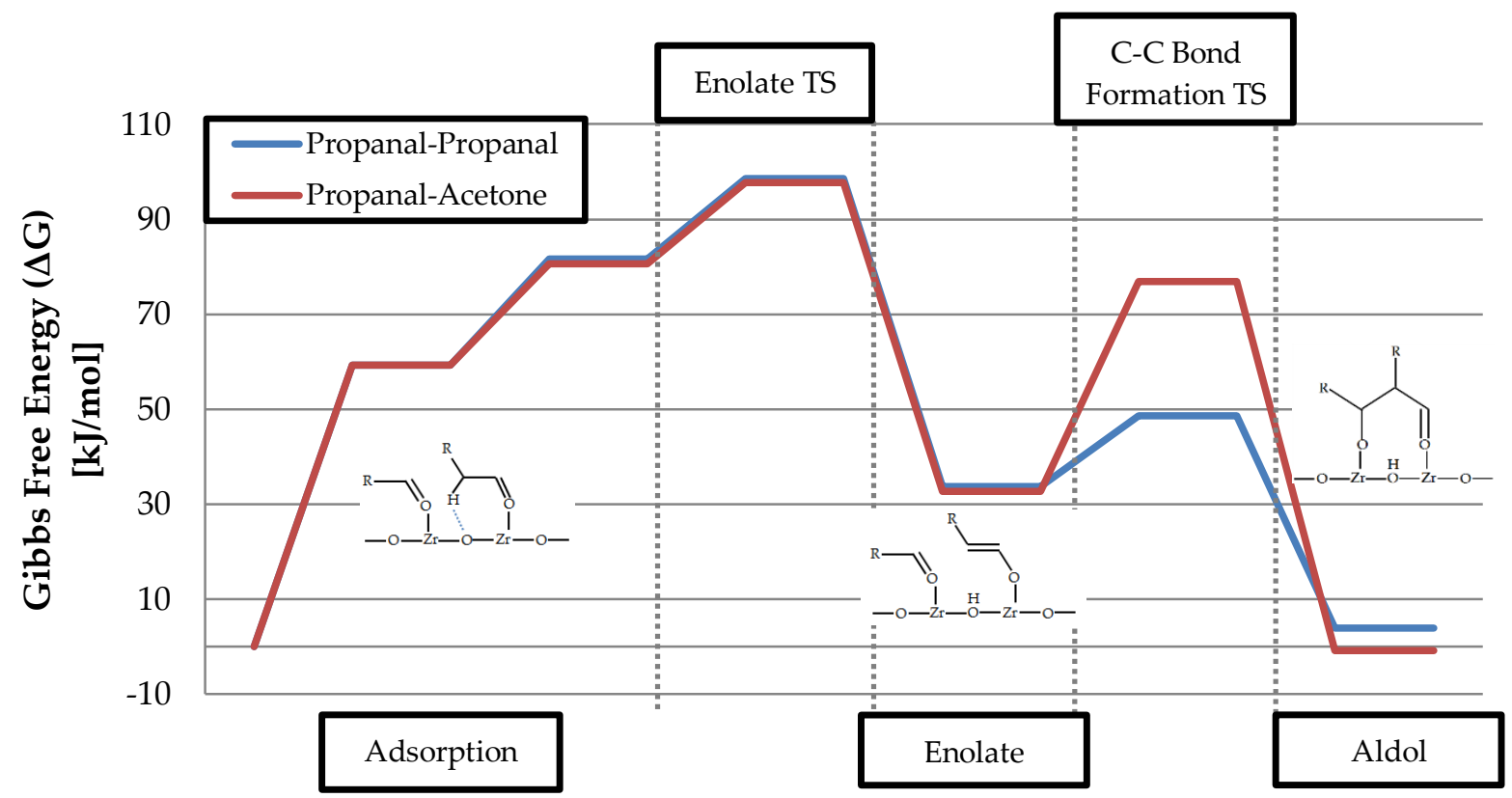

Figure 2.12.2: Relative energy diagram comparison of enolate formation and C-C bond formation steps for propanal-propanal (red) vs propanal-acetone (blue) over m- $\mathrm{ZrO}_{2}$.

A comparison of the intrinsic activation energies for propene self-coupling reveals comparable values across all of the surfaces examined, with intrinsic activation barriers of 15 , 14 , and $13 \mathrm{~kJ} / \mathrm{mol}$ for $\mathrm{m}-\mathrm{ZrO}_{2}, \mathrm{t}-\mathrm{ZrO}_{2}$, and $\mathrm{ZnO}$, respectively. These minor differences over the different surfaces likely stems from a lack of direct involvement of the oxide surface during the C-C coupling reaction, as both adsorbates remain fully coordinated to the surface and show no 
charge transfer to the surface along the reaction path. The only loss in bonding to the surface is the hydrogen bond between the secondary $\mathrm{C}_{\alpha---\mathrm{H}^{*}}$ interaction between the enolate intermediate and surface proton. While the Born-Haber analysis indicates a range of $\mathrm{C}-\mathrm{H}^{*}$ interactions for different oxide surfaces, this bonding appears to play only a minor role in C-C bond formation.

\subsection{Comparison of C-C Bond Formation and Enolate Formation Apparent Activation Energy}

As the Gibbs free energies for the overall reaction path are taken with respect to the gas phase reactants (Figure 2.13.1), it becomes clear that the effect of catalyst on enolate formation is far more critical than small differences that result in the $\mathrm{C}-\mathrm{C}$ formation step, as all of the oxide surfaces examined show significantly lower apparent free energy barriers for C-C bond formation than their barriers for enolate formation.

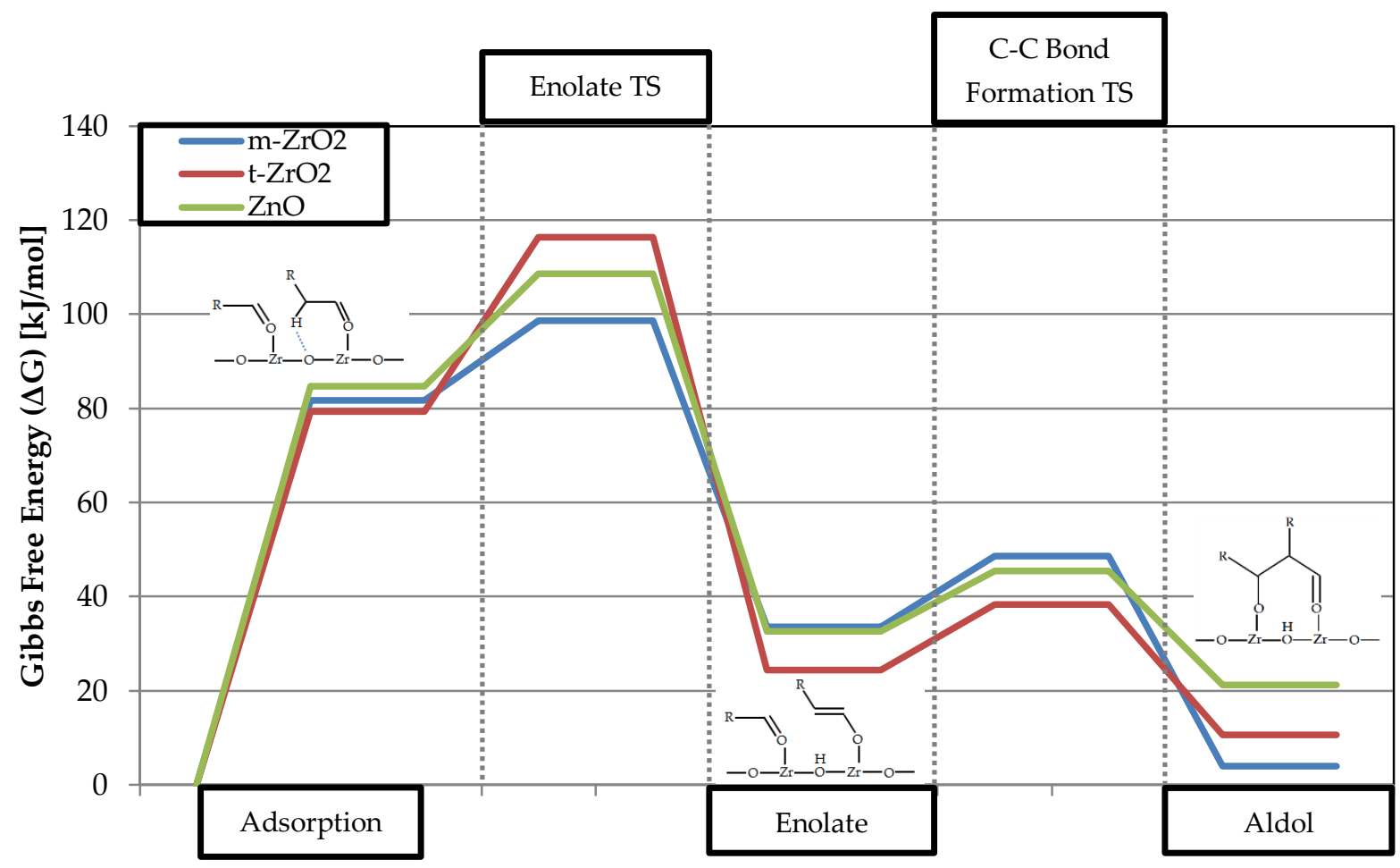

Figure 2.13.1: Overall Gibbs free energy diagram for propanal self-condensation over $\mathrm{m}-\mathrm{ZrO}_{2}$, $\mathrm{ZnO}$, and $\mathrm{t}-\mathrm{ZrO}_{2}$.

While both enolate formation and C-C bond formation yield low to moderate intrinsic activation energies, the moderately exothermic nature of the enolate formation step results in the forward $\mathrm{C}-\mathrm{C}$ bond formation step being more favorable than the reverse reaction. The heat 
of reaction for enolate formation of propanal on $\mathrm{m}-\mathrm{ZrO} 2$ is $-58 \mathrm{~kJ} / \mathrm{mol}$, which results in a total reverse barrier of $65 \mathrm{~kJ} / \mathrm{mol}$. This reverse reaction barrier is larger than the energetic barrier for C-C coupling of propanal enolate with any other reactant on $\mathrm{m}-\mathrm{ZrO}_{2}$, even in the case of the energetically difficult propanal/acetone cross-coupling case whose forward barrier is $44 \mathrm{~kJ} / \mathrm{mol}$ (Figure 2.12.2). As such, the kinetics for enolate formation will also control the overall kinetics for aldol condensation. However, once the enolate is formed, the intrinsic kinetics associated with the different $\mathrm{C}-\mathrm{C}$ bond formation steps dictate which reactants couple and provide insight into the resulting product distributions for mixed reactant streams.

\subsection{Concluding Remarks}

First-principle DFT calculations which explore the adsorption, activation and coupling of $\mathrm{C}_{2}-\mathrm{C}_{4}$ aldehydes and $\mathrm{C}_{3}-\mathrm{C}_{4}$ ketones over model $\mathrm{ZrO}_{2}$ and $\mathrm{ZnO}$ surfaces indicate that aldol condensation rates are controlled by the kinetics for enolate formation and that the greatest factors that control these rates are stabilization of the adsorbed enolate on the catalytic surfaces and the relative strength of the acid-base site pair. Stabilization potential inherent just to the gas phase molecules are limited to the length of the carbon backbone and stability differences inherent to aldehydes and ketones. However, when adsorbed to the weak acid-base site pairs on metal oxide surfaces, the close proximity of the acid and base site allows for stabilization through close proximity to the neighboring adsorbed proton via $\mathrm{O}^{*}-\mathrm{H}$ secondary interactions, and also through $\mathrm{Zr}^{*}-\mathrm{O}$ interactions with the surface itself via carbonyl oxygen chemisorption to the metal acid site. The relative Lewis base strength of the oxygen site shows a positive correlation to the activation energy for enolate formation and is consistent with conclusions declared in literature.

Conversely, the reaction kinetics for subsequent C-C coupling step is significantly more controlled by the reactant properties. Comparisons across self- and cross-coupling of $\mathrm{C}_{2}-\mathrm{C}_{4}$ aldehydes show a clear preference for the coupling of smaller molecules due to increased localization of negative charge at the enolate $\alpha$-carbon and decreased steric repulsion from the carbon tails. Comparison of propanal self-coupling to propanal/acetone cross-coupling activation energies also reveal a preference for aldehyde self-coupling for the same reasons 
stated above. However, comparisons of propanal self-coupling across different catalytic surfaces result in only slight differences in intrinsic activation barriers.

The overall apparent free energy diagram when taken with respect to the gas phase reveals enolate formation to be the more kinetically relevant step for aldol condensation. The combination of moderately exothermic heats of reaction for enolate formation and small energetic barriers for $\mathrm{C}-\mathrm{C}$ bond result in the reverse reaction from the enolate state being less active than the forward C-C bond formation step. These results also show the choice of catalytic surface plays a more important role on enolate formation than for subsequent C-C bond forming steps.

\section{$\underline{2.15 \text { References }}$}

[1] Burkell, J. L.; Fridgen, T. D.; McMahon, T. B., "Gas-phase acidities and sites of deprotonation of 2-ketones and structures of the corresponding enolates", Int. J. Mass Spectrom 2003, 227, 497-508.

[2] Chyall, L. J.; Brickhouse, M. D.; Schnute, M. E.; Squires, R. R., "Kinetic versus thermodynamic control in the deprotonation of unsymmetrical ketones in the gas phase", J. Am. Chem. Soc. 1994, 116 (19), 8681-8690.

[3] Finkelstein-Shapiro, D.; Buchbinder, A. M.; Vijayan, B.; Bhattacharyya, K.; Weitz, E.; Geiger, F. M.; Gray, K. A., “Identification of binding sites for acetaldehyde adsorption on titania nanorod surfaces using CIMS", Langmuir 2011, 27, 14842-14848.

[4] Raskó, J.; Keskés, T.; Kiss, J., “FT-IR and mass spectrometric studies on the interaction of acetaldehyde with $\mathrm{TiO}_{2}$-supported noble metal catalysts", Appl. Catal. A: Gen. 2005, 287, 244-251.

[5] Singh, M.; Zhou, N.; Paul, D. K.; Klabunde, K. J., "IR spectral evidence of aldol condensation: Acetaldehyde adsorption over $\mathrm{TiO}_{2}$ surface”, J. Catal. 2008, 260, 371-379.

[6] West, R. M.; Liu, Z. Y.; Peter, M.; Gartner, C. A.; Dumesic, J. A., “Carbon-carbon bond formation for biomass-derived furfurals and ketons by aldol condensation in biphasic system", J. Mol. Catal. a-Chem 2008, 296, 18-27. 
[7] Rekoske, J. E.; Barteau, M. A., "Kinetics, selectivity, and deactivation in the aldol condensation of acetaldehyde on anatase titanium dioxide", Ind. Eng. Chem. Res. 2011, $50,41-51$.

[8] Idriss, H.; Kim, K.; Barteau, M., “Carbon-carbon bond formation via aldolization of acetaldehyde on single crystal and polycrystalline $\mathrm{TiO}_{2}$ surfaces", J. Catal. 1993, 139, 119 133.

[9] Idriss, H.; Barteau, M. A., "Selectivity and mechanism shifts in the reactions of acetaldehyde on oxidized and reduced $\mathrm{TiO}_{2}(001)$ surfaces" Catal. Lett. 1996, 40, 147-153.

[10] Idriss, H.; Diagne, C.; Hindermann, J. P.; Kiennemann, A.; Barteau, M. A., “Reactions of acetaldehyde on $\mathrm{CeO}_{2}$ and $\mathrm{CeO}_{2}$-supported catalysts", J. Catal. 1995, 155, 219-237.

[11] Raskó, J.; Kiss, J., “Adsorption and surface reactions of acetaldehyde on $\mathrm{TiO}_{2}, \mathrm{CeO}_{2}$ and $\mathrm{Al}_{2} \mathrm{O}_{3}{ }^{\prime \prime}$, Appl. Catal. A: Gen. 2005, 287, 252-260.

[12] Zhang, G.; Hattori, H.; Tanabe, K., “Aldol addition of butyraldehyde over solid base catalysts", Bull. Chem. Soc. Jpn. 1989, 62, 2070-2072.

[13] Luo, S.; Falconer, J. L., “Aldol condensation of acetaldehyde to form high molecular weight compounds on $\mathrm{TiO}_{2}{ }^{\prime \prime}$, Catal. Lett. 1999, 57, 89-93.

[14] Ordomsky, V. V.; Sushkevich, V. L.; Ivanova, I. I., “Study of acetaldehyde condensation chemistry over magnesia and zirconia supported on silica", J. Mol. Catal. a-Chem 2010, $333,85-93$.

[15] Tanabe, K.; Holderich, W. F., “Industrial application of solid acid-base catalysts”, Appl. Catal. a-Gen 1999, 181, 399-434.

[16] Tanabe, K., “The future of acid-base catalysis”, Appl. Catal. A: Gen. 1994, 113, 147-152.

[17] Tanabe, K.; Yamaguchi, T., “Acid-base bifunctional catalysis by $\mathrm{ZrO}_{2}$ and its mixed oxides", Catal. Today 1994, 20, 185-198.

[18] Tanabe, K., "Are there any probe molecules suitable for measurement of acid-base pair sites?", Trends Anal. Chem. 1994, 13, 164-168.

[19] Climent, M. J.; Corma, A.; Iborra, S.; Velty, A., “Designing the adequate base solid catalyst with Lewis or Bronsted basic sites or with acid-base site pairs", J. Mol. Catal. aChem 2002, 182, 327-342. 
[20] Sad, M. E.; Neurock, M.; Iglesia, E., "Formation of C-C and C-O bonds and oxygen removal in reactions of alkanediols, alkanols, and alkanals on copper catalysts", J. Am. Chem. Soc. 2011, 133, 20384-20398.

[21] Tai, J.; Davis, R., "Synthesis of methacrylic acid by aldol condensation of propionic acid with formaldehyde over acid-base bifunctional catalysts", J. Catal. Today 2007, 123, 42-49.

[22] Young, Z.; Hanspal, S.; Davis, R., "Aldol condensation of acetaldehyde over titania, hydroxyapatite, and magnesia", ACS Catal. 2016, 6, 3193-3202.

[23] Wang, S.; Goulas, K.; Iglesia, E., “Condensation and esterification reactions of alkanals, alkanones, and alkanols on $\mathrm{TiO}_{2}$ : Elementary steps, site requirements, and synergistic effects of bifunctional strategies", J. Catal. 2016, 340, 302-320.

[24] Kresse, G.; Furthmüller, J., "Efficient iterative schemes for ab initio total-energy calculations using a plane-wave basis set", Phys. Rev. B 1996, 54, 11169-11186.

[25] Grimme, S., "Semiempirical GGA-type density functional constructed with a long-range dispersion correction", J. Comp. Chem. 2006, 27, 1787-1799.

[26] Audouze, C.; Jollet, F; Torrent, M.; Gonze, X., “Comparison between projector augmented-wave and ultrasound pseudopotential formalisms at the density-functional perturbation theory level", Phys. Rev. B 2008, 78, 035105.

[27] Tang, W.; Sanville, E.; Henkelman, G., “A grid-based Bader analysis algorithm without lattice bias", J. Phys-Condens. Mat. 2009, 21, 084204.

[28] Henkelman, G.; Uberuaga, B. P.; Jónsson, H., “A climbing image nudged elastic band method for finding saddle points and minimum energy paths", J. Chem. Phys. 2000, 113, 9901-9904.

[29] Henkelman, G.; Jónsson, H., "Improved tangent estimate in the nudged elastic band method for finding minimum energy paths and saddles points", J. Chem. Phys. 2000, 113, 9978-9985.

[30] Christensen, A.; Carter, E. A., "First-principles study of the surfaces of zirconia", Phys. Rev. B 1998, 58, 8050-8064.

[31] Eichler, A.; Kresse, G., "First-principles calculations for the surface termination of pure and yttria-doped zirconia surfaces", Phys. Rev. B 2004, 69, 045402. 
[32] Wander, A.; Harrison, N. M., “An ab initio study of $\mathrm{ZnO}(1010)$ ”, Surf. Sci. 2000 457, L342-L346.

[33] Bartmess, J. E.; Scott, J. A.; McIver, R. T., Jr, "Scale of acidities in the gas phase from methanol to phenol", J. Am. Chem. Soc. 1979, 101 (20), 6046-6056.

[34] Alconcel, L. S.; Deyerl, H. J.; Continetti, R. E., “Effects of alkyl substitution on the energetics of enolate anions and radicals", J. Am. Chem. Soc. 2001, 123 (50), 12675-12681.

[35] Chia, M.; Pagán-Torres, Y. J.; Hibbitts, D.; Tan, Q.; Pham, H. N.; Datye, A. K.; Neurock, M.; Davis, R. J.; Dumesic, J. A., "Selective hydrogenolysis of polyols and syslic ethers over bifunctional surface sites on rhodium-rhenium catalysts", J. Am. Chem. Soc. 2011, $133,12675-12689$. 


\section{CHAPTER 3: Alkene Oligomerization in Zeolites}

\subsection{Alkene Oligomerization of Light Olefins within Zeolites}

Acid catalysts are also known to be very effective in catalyzing C-C bond formation. The Brønsted acid sites in zeolite catalysts readily catalyze a range of different hydrocarbon transformations, including the catalytic coupling of small olefins derived from other oxygenate transformations such as alcohol dehydration processes. Zeolites are used industrially to catalyze a wide range of hydrocarbon transformations important in the production of fuels and chemicals from biomass and other feedstocks, including the conversion of methanol-to-olefins, isomerization, alkylation and light alkene oligomerization [1]. Alkene oligomerization is an important process in the formation of $\mathrm{C}-\mathrm{C}$ bonds and hydrocarbon fuel production that is actively carried out over Brønsted acid sites in zeolites. The mechanism for oligomerization is thought to proceed via the formation of a charged carbenium ion intermediate via protonation of the olefin by zeolite Brønsted acid site followed by a C-C coupling reaction which involves the attack of the electrophilic carbon center of the carbenium ion on the nucleophilic carbon of the adsorbed olefin via the electron-rich $\pi$-bond. Both aldol condensation and alkene oligomerization reactions appear to show similar characteristics in that they involve the formation of a charged intermediate that attacks a carbon center of a second co-adsorbed molecule.

Acid catalyzed transformations typically involve the formation of positively charged carbenium ions that add to olefins, abstract a hydride, undergo structural isomerization or crack to form lighter products. The formation and activity of carbenium ion intermediates, in particular, has been studied extensively as carbenium intermediates are very often the most abundant surface species in zeolites during a number of different hydrocarbon transformations. An experimental study by Kirisci, Pálinkó, and Kollár found that, under reaction conditions, all of the available Brønsted acid sites within zeolites were replaced with absorbed carbenium ions to form the surface alkoxides. They studied hydrocarbon adsorption, carbenium ion formation, and build-up of carbonaceous residues in HY-FAU zeolite at moderate temperatures using UVVis spectroscopy [2]. Their results observed a shift in adsorption bands from alkene to unsaturated alkenyl ions, signifying the formation of adsorbed alkenyls in place of Brønsted 
acid sites. At higher temperatures, the adsorbed alkenyls began acting as Lewis acids for additional adsorption via oligomerization and reabsorption of the dimerized alkenyls. Their results illustrate the prevalence of carbenium ions within zeolites during hydrocarbon transformation reactions.

Additional studies have also noted the formation of carbenium ions upon solid acid catalysts, and have illustrated the importance of carbenium ion stabilization for hydrocarbon upgrading. Janik, Davis, and Neurock studied the formation and stabilization of tertiary carbenium ions over phosphotungstic acid for acid-catalyzed hydrocarbon conversion reactions [3]. An iso-density surface plot of the carbenium ion over the heteropoly acid (HPA) convex catalytic surface revealed very little electron density overlap, and an analysis of the carbenium ion interaction energy with the HPA found that the attractive coulombic energy accounted for the vast majority of the energy, with minor repulsive non-coulombic energy from the carbenium ion's proximity to the HPA surface. A follow-up study examined hydride transfer and alkylation of isobutene over a phosphotungstic acid catalyst using periodic density functional theory (DFT) [4]. They showed that both reactions proceed through a transition state converting the adsorbed alkyl to a carbenium ion intermediate. In the case of hydride transfer, the carbenium ion stability is unaffected by the physisorbed alkane, but in the case of alkylation the carbenium ion is stabilized through interactions with the physisorbed alkene.

The adsorption and catalytic activity for these reactions can be significantly influenced by the size and topology of the zeolite. Derouane proposed that shape selectivity in zeolites was governed by an enthalpic "nest effect", in which molecules and the surrounding zeolite framework optimized the attractive Van der Waals (vdW) interactions that affect intrinsic catalytic activity [5]. Evidence for this effect was cited for the alkylation of aromatics in ZSM-5 and paraffin cracking where the optimal zeolite pore size diameter for the maximum turnover frequency (TOF) for paraffin cracking for various zeolites was found to be 5.8-6.0 $\AA$, which is very close to the critical dimension of the transition state. In subsequent studies, Derouane, André, and Lucas proposed a simple vdW model to describe the secondary interactions of a molecule with a curved surface, and tested its validity using experimental physisorption data and catalytic kinetics for cracking of n-pentane at $450 \mathrm{C}^{\circ}$ reported in literature at the time [6]. 
The model illustrated the importance of these physical effects in predicting the turnover frequencies for the catalytic cracking of alkanes and other catalytic systems, which could not be explained solely by acid-site strength and density. The excellent quantitative agreement between the model and the observed experimental TOF suggests that the differences in activity arise from the "nesting effect" rather than variations in acid strength. Derouane et al. also analyzed possible curvature effects for adsorption and concentration of species near the active sites using a simple vdW model and pore geometry [7] and showed a direct correlation between the pore cavity size of the zeolite and molecular physisorption. Their model successfully captured the influence of the zeolite as a "molecular trap" when the pore radius approached the sorption distance. It also illustrated an energetic penalty for the molecule to escape the zeolite walls to a free-floating state at the direct center of the pore. These results emphasize that the curvature, which affects the physisorption energetics and local concentration at the zeolite catalytic site, can play an important role in controlling the adsorption and catalytic behavior of different molecules. This effect of confinement continues to be noted in more recent literature on hydrocarbon transformation in zeolites, including work by Lucas et al. which predicted the creeping diffusion of benzene along the HY zeolite via equations involving the vdW attraction and interatomic forces [8], as well as experimental work by Tanchoux et al. whose results on conversion rates of isomerization of n-1-hexene to n-2-hexene illustrate a clear dependence on pore size [9].

This effect of solvation has also been noted in the realm of hydrocarbon cracking, which is the reverse of oligomerization. Luo et al. analyzed n-hexane cracking at high pressures for $\mathrm{H}$ ZSM-5, H-BEA, and H-MOR zeolites and studied selectivities and rate expressions of those reactions [10]. It was found that the larger pore zeolites, H-MOR and H-BEA, primarily produced isomerization products at high pressures at $>50 \%$ selectivity, but very few olefin products, and yielded a non-Langmuir Hinshelwood rate expression, whereas H-ZSM-5 exhibited higher selectivities to olefins and matched a simple Langmuir-Hinshelwood rate expression for the pressures and temperatures tested. It was suggested that the large discrepancy in selectivity derives from steric constraints where the pore-size restrictions of $\mathrm{H}$ ZSM-5 suppresses olefin oligomerization to aromatic molecules, which minimizes the coking 
effects observed in MOR and BEA. This is also supported by larger zeolites promoting hydride transfer step as evidenced by increased isomerization products, in which hydride transfer activity benefitted from the increased volume. A review paper by Smit and Maesen analyzed the extensive computational work already done with regard to alkane adsorption, diffusion, and shape selectivity of hydrocarbon cracking in zeolites. They report the influence of the zeolite framework on adsorption and diffusion, but more work is still needed to quantitatively predict the effects of zeolite structure on shape selectivity and reaction kinetics for hydrocarbon transformation [11].

The attractive as well as the repulsive interactions that occur between the reactants and transition states and zeolite that occur in alkene oligomerization can significantly influence the observed catalytic reactivity and selectivity for different reactant/zeolite combinations. Pantu, Boekfa, and Limtrakul analyzed the physisorption of alkanes and alkenes into H-MOR and HFAU using the Our-own-N-layer Integrated molecular Orbital + molecular Mechanics theoretical method (ONIOM) [12]. Using the adsorption energies for alkanes as a measure for method accuracy, the ONIOM theoretical method was applied for alkene adsorption energies, which could not be determined experimentally. The predicted alkane adsorption energies found good agreement with experimental values, and analysis of alkene physisorption over the Brønsted-acid site found that vdW interactions contributed $50 \%$ of the calculated adsorption energy. Corma, Martinez, and Doskocil analyzed the effect of acid-site density and mesoporisity for propene and 1-pentene oligomerization to fuels in ZSM-5 [13]. Their results found that propene oligomerization demonstrated a higher higher activity and lower deactivation in ZSM-5 catalysts with higher Brønsted acid-site density and smaller crystallites, but found the opposite was true for 1-pentene oligomerization over the same catalysts. Mlinar et al. analyzed the effect of Si/Al ratios on propene oligomerization in H-MFI catalysts [14]. Insitu FT-IR spectroscopy showed that all of the free Brønsted sites present were consumed within the first minute on stream. Both the $\mathrm{CH}_{2}$ and $\mathrm{CH}_{3}$ adsorption bands grew with time, thus suggesting the formation of adsorbed oligomerization products. Kinetic analysis found that lower Si/Al ratios, indicating increased site density in H-MFI, yielded lower activity and higher selectivity towards dimer oligomerization products. This suggests there are steric 
constraints from adsorbed oligomers on nearby active sites. The possibility of steric constraints was also supported by ab initio QM/MM analysis carried out by Mlinar et al. They showed that the number of nearest neighbors did not significantly influence the activation energies for oligomerization of propene with 2-propoxide, but significantly increased trimer oligomerization activation energies of propene with $\mathrm{C}_{6}$ dimerized alkenes formed in situ.

Alkene oligomerization can be significantly influenced by the interactions of the reactant and the transition state with the zeolite framework. These states can be significantly stabilized by their interaction with the zeolite framework, but little is known about the extent of this stabilization and the relative impact with respect to other contributing factors. The work herein analyzes alkene oligomerization of propene and isobutene with ethoxy, 2-propoxy and t-butoxy adsorbed alkoxides within MFI, TON and MOR.

\subsection{Catalytic Mechanism for Alkene Oligomerization in Zeolites}

Alkene oligomerization in zeolites (Figure 3.2.1) proceeds via the adsorption of an alkene to a Brønsted acid site within the zeolite and subsequent protonation of the olefin to form a positively charged carbenium ion that binds to the Al- $\mathrm{O}^{*}$ site as an alkoxide. A second alkene then physisorbs into the zeolite and is stabilized near the adsorbed alkoxy via secondary interactions with the adsorbed alkoxide and surrounding zeolite framework. Carbon-carbon bond formation proceeds via the activation of the $\mathrm{RCH}_{x}-\mathrm{OZ}$ bond to reform the carbenium ion between the negatively charged $\mathrm{Al}-\mathrm{O}^{*}$ site and the electron-rich $\pi$-bond of the physisorbed alkene. The positively charged carbon center attacks the alkene $\pi$-bond and forms a C-C bond with the less substituted carbon, yielding the dimerized carbenium ion intermediate that is stabilized by secondary interactions with the surrounding zeolite framework. The resulting carbenium ion can then undergo rotation and chemisorb back onto the Al-O* site. It can subsequently deprotonate at the Al-O* site to form the coupled alkene and reform the Brønsted acid site, or react with another physisorbed reactant via hydride transfer from the physisorbed

species to form the coupled alkane product, leaving the smaller carbenium ion adsorbed on the Al---O* site in its place. 


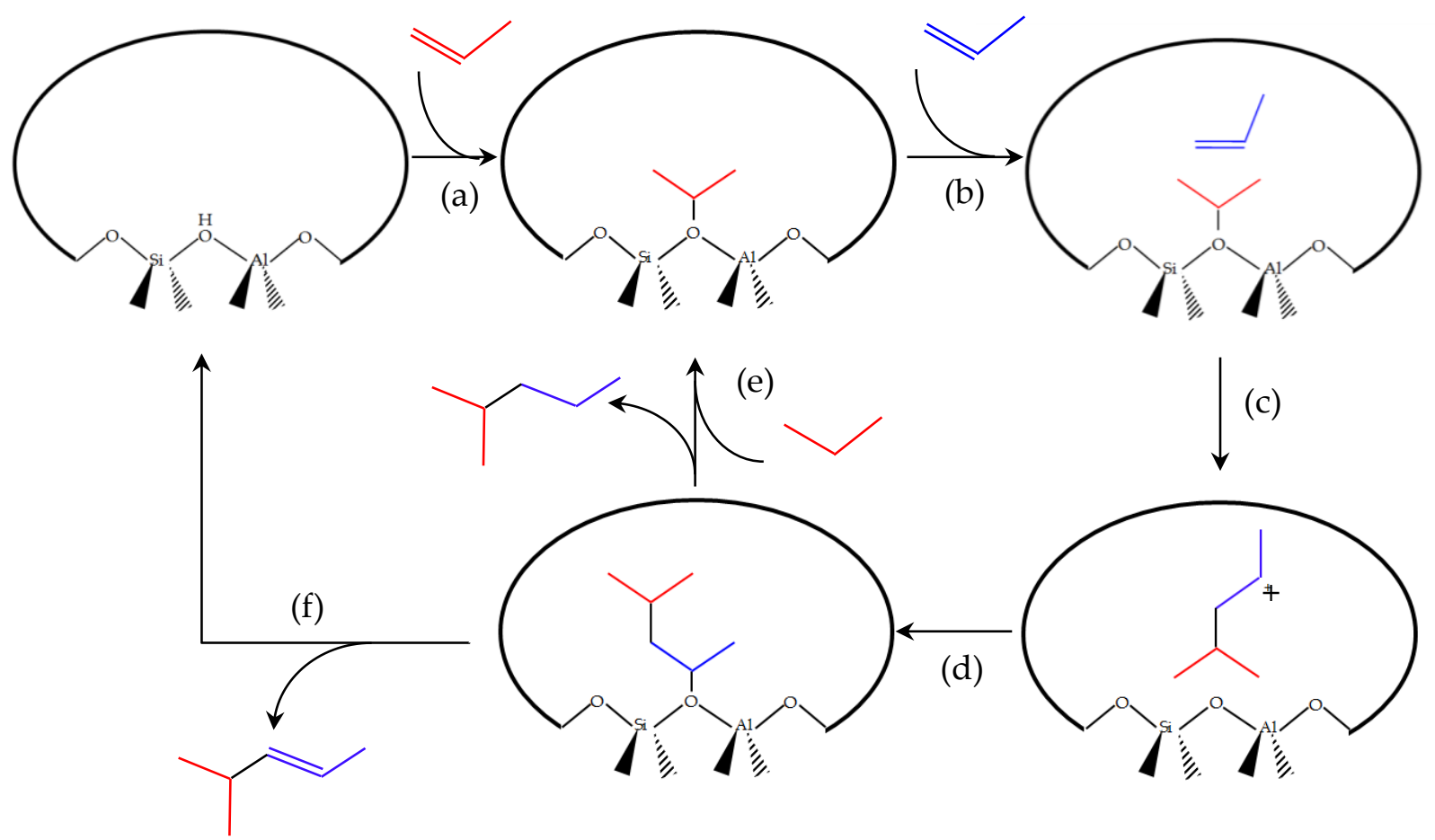

Figure 3.2.1: Reaction scheme for alkene oligomerization detailing the elementary reactions involved including: (a) alkene adsorption and protonation to form the surface alkoxy intermediate, (b) 2nd alkene physisorption, (c) oligomerization via the reaction of the alkene and alkoxy, (d) rotation and chemisorption of the resulting alkyl intermediate, (e) hydride transfer to the alkyl and alkane product desorption, and (f) deprotonation and alkene product desorption.

\subsection{Alkene Oligomerization Computational Methods}

All calculations reported herein were carried out using periodic dispersion-corrected density functional theory (DFT-D2) calculations as implemented in the Vienna ab initio Simulation Package (VASP) [15]. The effects of dispersion were accounted for through the use of semi-empirical Grimme-D2 corrections [16]. The Perdew-Burke-Ernzerhof (PBE) exchangecorrelation functional was used to calculate nonlocal gradient corrections to exchange and correlation energies. Projector-augmented wave (PAW) pseudopotentials were used to describe core electrons [17]. Valence electrons were described with a plane-wave basis set with an energy cutoff of $396 \mathrm{ev}$. The wave functions and charge density were converged to within $10^{-6}$ $\mathrm{eV}$. Due to the large unit-cell sizes and the localized nature of the valence electrons of the 
zeolites, the sampling of the Brillouin zone was done at the $\gamma$ point only. All atoms were allowed to relax during geometric optimization with a force criteria and energy criteria for optimization set at $0.05 \mathrm{eV} / \AA$ and $10^{-6} \mathrm{eV}$, respectively. Atomic charges were analyzed using the Bader charge analysis [18], which describes the charge distribution by partitioning of the electron density.

The saddle points were initially located using the nudged elastic band method (NEB) to map out the reaction coordinate $[19,20]$. Thirty two evenly spaced points were chosen along the reaction coordinate and the structures at each of these points were minimized until the forces perpendicular to the reaction path converged to within $0.25 \mathrm{eV} / \AA$. The minimum energy reaction path and the approximate transition states were subsequently used as input to isolating more refined transition states using the dimer method [20]. The dimer calculations were converged until the forces perpendicular to the reaction mode were less than $0.05 \mathrm{eV} / \AA$.

\subsection{Calculation of Gibbs Free Energy of Adsorption}

The free energy of adsorption was determined using the Henry's adsorption constant for alkene adsorption into an empty zeolite. Henry's constant was calculated at the zero-coverage limit using the Widom insertion method and configurational-bias Monte Carlo algorithm in a frozen zeolite [21]. The TraPPE-UA and TraPPE-zeo potentials were used to model the interactions between the chemisorbed alkoxides, physisorbed alkenes, and zeolite $\mathrm{SiO}_{2}$ framework [22-25]. These potentials are derived from molecular-mechanics force fields, consisting of Lennard-Jones 12-6 coulombic terms and utilize the Lorentz-Berthelot mixing rule for unlike interactions.

The Henry's constant $\left(\mathrm{K}_{\mathrm{H}, \mathrm{cp}}\right)$, zeolite mass per unit cell (munit), volume of the unit cell ( $\mathrm{V}_{\text {unit }}$ ), and ideal gas law were used to calculate the dimensionless adsorption equilibrium constant using equations 1-3. Equation 1 multiplies Henry's constant by the mass per unit cell to calculate the number of moles per pressure per unit cell $(n / P)$, which is then used together with ideal gas law in order to calculate the dimensionless equilibrium adsorption constant (Kequilibrium) shown in Equation 2. Substitution of equation 1 into equation 2 yields the overall equation for calculating equilibrium adsorption constant from Henry's constant. 


$$
\begin{gathered}
K_{H, c p}\left[\frac{\mathrm{mol}}{k g * M P a}\right] * m_{\text {unit }}\left[\frac{\mathrm{kg}}{\text { unit cell }}\right]=\frac{n}{P}\left[\frac{\mathrm{mol}}{M P a * \text { unit cell }}\right] \\
K_{\text {equilibrium }}=\frac{n}{P} * \frac{R T}{V_{\text {unit }}} \\
K_{\text {equilibrium }}=K_{H, c p} * m_{\text {zeolite }} * \frac{V_{\text {unit cell }}}{R T}
\end{gathered}
$$

With the adsorption equilibrium constant, the Gibb's free energy of adsorption is then calculated shown in equation 4.

$$
\Delta G_{a d s}=-R T \ln \left(K_{\text {equilibrium }}\right)
$$

\subsection{Calculation of Entropy for Free Energies of Activation}

The entropy of the apparent reaction was calculated using the vibrational contributions to entropy for the zeolite systems at the transition state and then compared to the entropy of the referenced zeolite system with an adsorbed alkoxy and gas phase molecule. The transition state was described with only vibrational frequencies due to the tightly bound nature of the transition state within the zeolite, and we expect any translational or rotational contributions to be minimal as movement away from the $\mathrm{Al}-\mathrm{O}^{*}$ site would drive the reaction towards the reactant or product state. The reactant state with adsorbed alkoxy and gas phase alkene was measured using vibrational frequencies of the zeolite system as well as translational, rotational, and vibrational contributions to entropy for the gas phase alkene.

The vibrational partition function was calculated using the first vibrational mode as reference:

$$
q_{V}=1 /\left(1-\exp \left(\frac{h c v}{k_{B} T}\right)\right)
$$

The vibrational, translational, and rotational contributions to the partition function of the alkene gas molecule $\left(\mathrm{Q}_{\mathrm{gas}}\right)$ were calculated using ideal gas partition function expressions at 1 atm and 298.15 Kelvin, where $m_{\text {gas }}$ is the mass of the alkene and $V_{\text {gas }}$ is the volume of the ideal gas. 


$$
Q_{g a s}=q_{g a s}=V_{g a s} *\left(\frac{2 \pi m_{g a s} k_{B} T}{h^{2}}\right)^{3 / 2}
$$

The apparent change in entropy of reaction $\left(\Delta S^{*}{ }_{\text {app }}\right)$ was then calculated via the following equations, where $\mathrm{Q}^{\neq} \mathrm{v}$ is the vibrational partition function at the transition state, $\mathrm{Q} v, \mathrm{R}$ is the vibrational partition function of the zeolite at the reactant state.

$$
\begin{gathered}
Q_{V}=\prod q_{V} \\
S=R \ln \left(Q_{V}\right) \\
\Delta S_{a p p}^{\neq}=R \ln \left(\frac{Q_{V}^{\neq}}{Q_{V, R} Q_{g a s}}\right)
\end{gathered}
$$

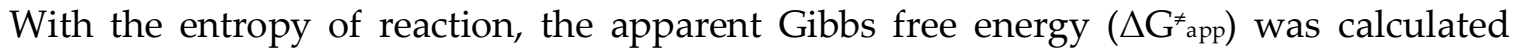
using the following equation at $\mathrm{T}=600 \mathrm{~K}$.

$$
\Delta G_{a p p}^{\neq}=\Delta H^{\neq}-T \Delta S^{\neq}
$$

The intrinsic Gibbs free energy describing the change in free energy from the physisorbed alkene and chemisorbed alkoxy state to the dimerized transition state was then calculated by adding the apparent free energy with the free energy of adsorption.

$$
\Delta G_{i n t}^{\neq}=\Delta G_{a d s}+\Delta G_{a p p}^{\neq}
$$

\section{$\underline{3.6 \mathrm{TON}, \mathrm{MFI} \text {, and MOR Structure Models }}$}

Initial zeolite models and atomic coordinates for MFI, MOR, and TON, were imported from Accelrys Materials Studio structure library [26] and from the database of zeolite structures [27] (Figure 3.6.1). All zeolite models studied herein contain a single Al atom within the theoretical supercell and placed at the intersection of two crossing channels where applicable. The effect of neighboring $\mathrm{Al}$ or increased $\mathrm{Al}$ concentration was not studied. 

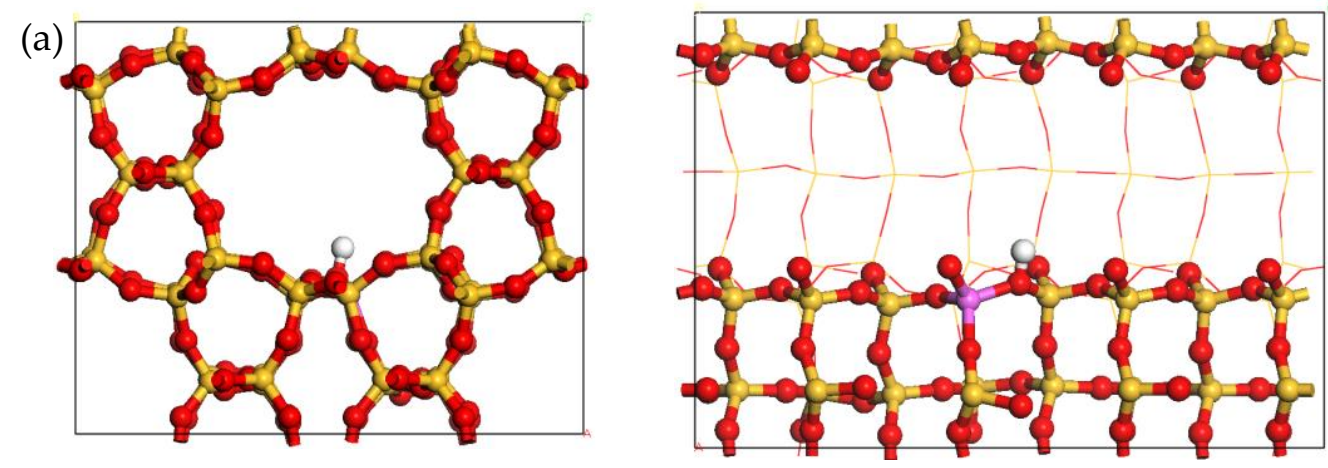

$\underline{\text { TON }}$
PLD $=5.11 \AA$
$\mathrm{LCD}=5.71 \AA$

(b)
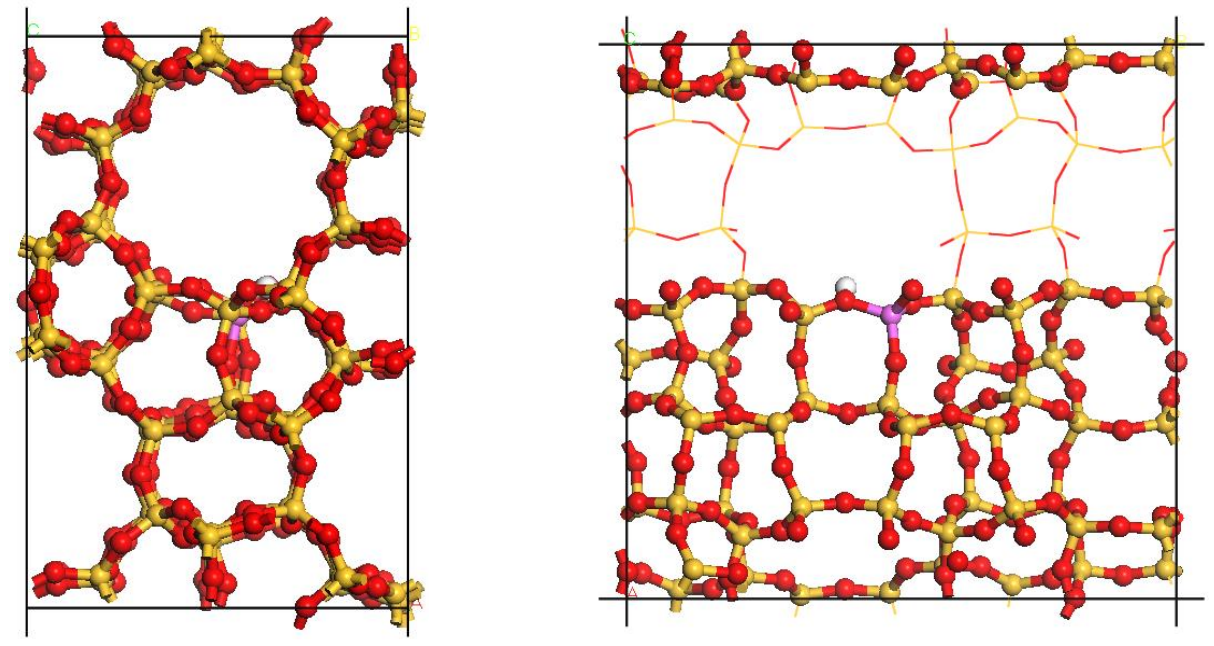

$\underline{\text { MFI }}$

$\mathrm{PLD}=4.7 \AA$

$\mathrm{LCD}=6.36 \AA$
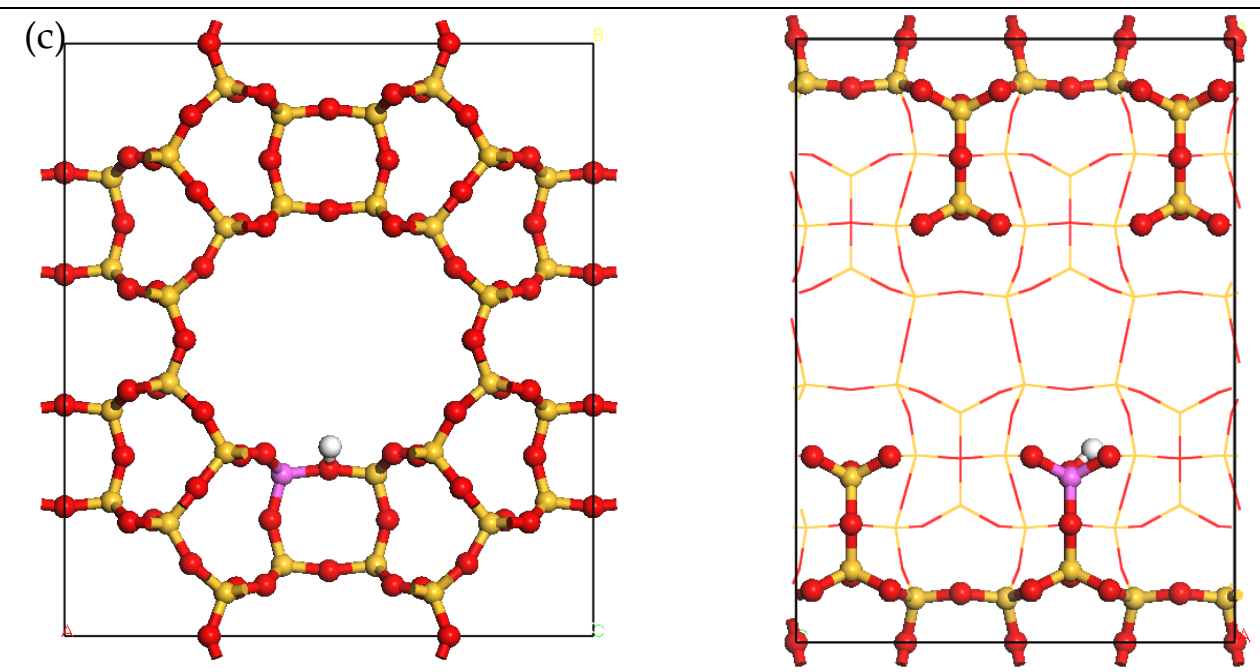

$\underline{\mathrm{MOR}}$

$\mathrm{PLD}=6.45 \AA$

$\mathrm{LCD}=6.7 \AA$

Figure 3.6.1: Front and side view of the zeolite structure and the pore limiting diameter (PLD) and largest cavity diameter (LCD) for (a) TON, (b) MFI, and (c) MOR zeolite models. 
MFI structure is composed of single unit cell and contains 288 atoms with fixed lattice parameters set at $\mathrm{a}=20.022 \AA, \mathrm{b}=19.899 \AA, \mathrm{c}=13.383 \AA$ and $\alpha=\beta=\gamma=90^{\circ}$. An $\mathrm{Al}$ atom was substituted in place of a Si atom at the intersection between the 12-member ring straight channel and 12-member ring perpendicular oscillating channel for the reasons stated previously. TON structure is comprised of a $(1 \times 1 \times 4)$ supercell, with the unit cell expanded in the direction along the zeolite channel. The final supercell contains 288 atoms with fixed lattice parameters of $\mathrm{a}=$ 13.859 $\AA, \mathrm{b}=17.420 \AA, \mathrm{c}=20.152 \AA$ and $\alpha=\beta=\gamma=90^{\circ}$. As there is only a single 12-member ring straight channel within TON, the $\mathrm{Al}$ atom was substituted in place of a Si atom on the edge with lowest curvature to allow for better fit of the alkoxy and alkene within the TON zeolite. MOR structure is composed of $(1 \times 1 \times 2)$ supercell, with the unit cell expanded in the direction along the zeolite channel. The final supercell contains 314 atoms with fixed lattice parameters at $\mathrm{a}=$ $18.094 \AA, \mathrm{b}=20.516 \AA, \mathrm{c}=15.048 \AA$ and $\alpha=\beta=\gamma=90^{\circ}$. An $\mathrm{Al}$ atom was exchanged for a Si atom on the edge with lowest curvature as this location places the $\mathrm{Al}$ next to the 8-member ring pockets within the 14-member ring straight channel, and is consistent with $\mathrm{Al}$ atom placement in comparison to TON and MFI. Reactions were studied at the intersections as these intersections provide the largest amount of space available for alkene oligomerization.

\subsection{Gas Phase Calculations}

Gas phase calculations for alkene oligomerization were carried out to determine the intrinsic differences in energy related to the gas phase stability of the reactants, carbenium ion intermediates and products before the influence of the zeolite framework (Figure 3.7.1). The gas phase activation of the $\mathrm{C}^{*}-\mathrm{OZ}$ bond to the resulting carbenium ion and the $\mathrm{C}^{*}-\mathrm{C}$ bond formation energy between the carbenium ion and alkene reactant were calculated separately, and then added together to obtain the total energy for alkene oligomerization as these two steps occur in a single concerted step in the mechanism (Tables 3.7.2-3.7.4). $\mathrm{C}^{*}-\mathrm{O}$ bond activation energy was calculated using a simple single Al-O* site cluster shown in Figure 3.7.1 (Table 3.7.2). Hydrogen which is used to cap the dangling Si sites takes on the form of a hydride. As such, the basicity of the $\mathrm{Al}-\mathrm{O}^{*}$ site is decreased in comparison to the acidity of the Al-O* site in the periodic zeolite structure. The overall oligomerization $\Delta \mathrm{E}_{\mathrm{rxn}}$ values reported here likely underestimate 
the $\mathrm{C}^{*}$---O activation barrier, but the resulting differences between alkoxides are still qualitatively conclusive.
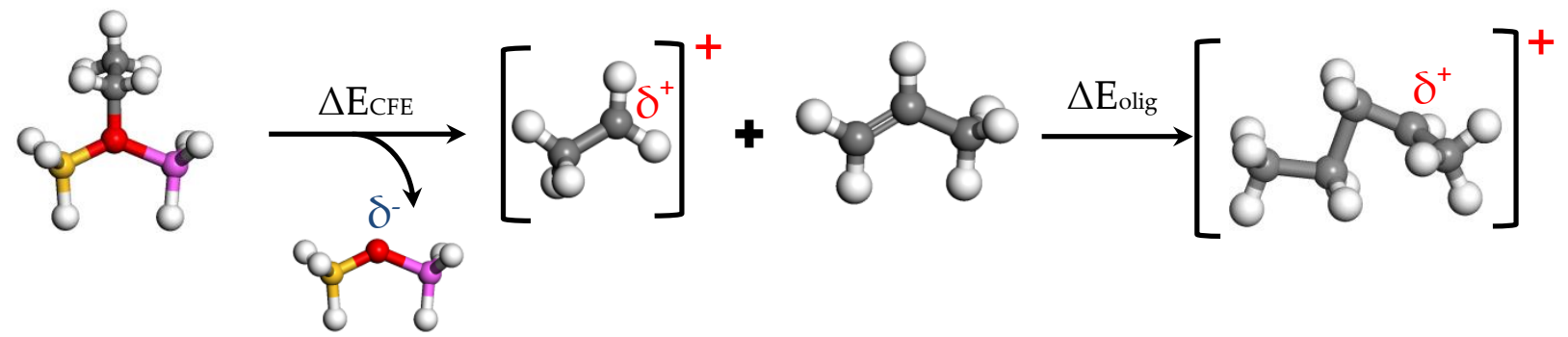

Figure 3.7.1: Reaction scheme for gas phase analysis of alkene oligomerization, measuring

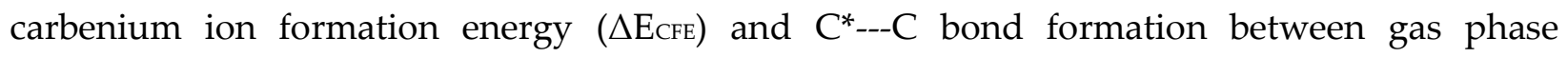
carbenium ion and alkene $(\Delta$ Eolig $)$.

Table 3.7.2: The carbenium ion formation energy $\left(\Delta \mathrm{E}_{\mathrm{CFE}}\right)$ for different $\mathrm{C}_{2}-\mathrm{C}_{4}$ bound alkoxides required to break the $\mathrm{C}^{*}-\mathrm{OZ}$ bond to form a free carbenium ion and the negatively charged zeolite lattice.

\begin{tabular}{|ll|l|}
\hline Dealkylation of bound alkoxide & $\begin{array}{l}\mathrm{C}^{*} \text {-O Activation } \\
{[\mathrm{kJ} / \mathrm{mol}]}\end{array}$ \\
\hline Ethoxy & {$\left[\mathrm{AlO}^{*}-\mathrm{CH}_{2} \mathrm{CH}_{3}\right]$} & 744 \\
\hline 2-Propoxy & {$\left[\mathrm{AlO}^{*}--\mathrm{CH}\left(\mathrm{CH}_{3}\right)_{2}\right]$} & 644 \\
\hline t-Butoxy & {$\left[\mathrm{AlO}^{*}--\mathrm{C}\left(\mathrm{CH}_{3}\right)_{3}\right]$} & 570 \\
\hline
\end{tabular}

The $\mathrm{C}^{*}-\mathrm{O}$ activation calculations show that t-butoxy has the lowest energy barrier for carbenium ion formation while ethoxy has the highest. This reflects the intrinsic stability of the resulting carbenium ion intermediate, as the tertiary carbenium ion center on t-butoxy is better able to delocalize the resulting positive charge in comparison to the secondary and primary carbenium ion centers for 2-propoxy and ethoxy, respectively. The increase in substitution from primary to secondary carbon center for ethoxy to 2-propoxy decreased the required energy by $100 \mathrm{~kJ} / \mathrm{mol}$ emphasizing the large instability inherent to the primary carbon. An increase in substitution from secondary to tertiary carbenium ion center also yields a significant, but smaller, decrease in the energy required for $\mathrm{C}^{*--} \mathrm{O}$ activation at $74 \mathrm{~kJ} / \mathrm{mol}$. 
Table 3.7.3: $\quad C^{*}---C$ bond formation energy between $C_{2}-C_{4}$ carbenium ions and propene or isobutene $(\Delta$ Eolig $)$.

\begin{tabular}{|l|l|l|}
\hline $\begin{array}{l}\mathrm{C}---\mathrm{C} \text { Bond Formation } \\
{[\mathrm{kJ} / \mathrm{mol}]}\end{array}$ & Propene & Isobutene \\
\hline Ethyl cation $\quad\left[\mathrm{CH}_{3}-\mathrm{CH}_{2}(+)\right]$ & -249 & -302 \\
\hline 2-Propyl cation $\left[\left(\mathrm{CH}_{3}\right)_{2}-\mathrm{CH}(+)\right]$ & -154 & -203 \\
\hline t-Butly cation $\left[\left(\mathrm{CH}_{3}\right)_{3}-\mathrm{C}(+)\right]$ & -92 & -140 \\
\hline
\end{tabular}

The stability of the carbenium ion center also plays a role in the calculated $\mathrm{C}^{*}-\mathrm{C}$ bond formation step, where ethoxy results in the reaction with the greatest exothermicity while $t$ butoxy yields the lowest exothermicity. The ethoxy is favored over the t-butoxy by roughly 60 $\mathrm{kJ} / \mathrm{mol}$. The difference in the $\mathrm{C}^{*}-\mathrm{C}$ bond formation energy reflects the reactivity of the carbenium ion center, as the least stable primary carbenium ion centers yield the most exothermic reaction energies in comparison to the more stable secondary or tertiary carbenium ion centers for both isobutene and propene $\mathrm{C}^{*}-\mathrm{C}$ bond formation. It is possible that the diminishing returns for $\mathrm{C}^{*}-\mathrm{C}$ bond formation is partially affected by intermolecular steric repulsion between highly substituted carbenium ion carbon centers with the primary reactive carbon site on the alkene, which was observed in the case of $C^{*}-C$ bond formation for aldol condensation. Further evidence, however, is required in order to fully assess this hypothesis.

With regards to the effect of the alkene, the simulation results show that $\mathrm{C}^{*}-\mathrm{C}$ bond formation with isobutene is consistently more favorable than the $C^{*}-C$ formation with propene by approximately $50 \mathrm{~kJ} / \mathrm{mol}$ for all of the alkoxides examined. This is due to the stability of the positive charge in the resulting dimerized intermediate, as oligomerization with isobutene places the final positive charge at a tertiary carbon while oligomerization with propene places the final positive charge at a secondary carbon. A comparison of both effects reveals that the stability of the reacting carbenium ion intermediate and that of the resulting dimerized intermediate hold similar importance to the overall energetics, and show that the more substituted reactants are more active for oligomerization, with tbutoxy-isobutene oligomerization being the most active and ethoxy-propene oligomerization being the least. 
Table 3.7.4: The combined gas phase energy $\left(\Delta\right.$ Erxn) for alkene oligomerization between $C_{2}-C_{4}$ carbenium ions and propene or isobutene via summation of dealkylation $\left(\Delta \mathrm{E}_{\mathrm{CFE}}\right)$ and $\mathrm{C}-\mathrm{C}^{*}$ bond formation $(\Delta$ Eolig $)$ energies

\begin{tabular}{|l|l|l|}
\hline Oligomerization & $\begin{array}{l}\text { Propene } \\
\Delta \text { Erxn }[\mathrm{kJ} / \mathrm{mol}]\end{array}$ & $\begin{array}{l}\text { Isobutene } \\
\Delta \text { Erxn }[\mathrm{kJ} / \mathrm{mol}]\end{array}$ \\
\hline Ethoxy $\left[\mathrm{AlO}^{*}-\mathrm{CH}_{2} \mathrm{CH}_{3}\right]$ & 495 & 442 \\
\hline 2-Propoxy $\left[\mathrm{AlO}^{*}-\mathrm{CH}\left(\mathrm{CH}_{3}\right)_{2}\right]$ & 490 & 441 \\
\hline t-Butoxy $\left[\mathrm{AlO}^{*}--\mathrm{C}\left(\mathrm{CH}_{3}\right)_{3}\right]$ & 478 & 430 \\
\hline
\end{tabular}

Total oligomerization energy calculations reveal that, for both propene and isobutene oligomerization, the more substituted alkoxides yield lower oligomerization energies, with propene-ethoxy oligomerization resulting in the highest energy at $495 \mathrm{~kJ} / \mathrm{mol}$ and propene-tbutoxy oligomerization having the lowest energy at $478 \mathrm{~kJ} / \mathrm{mol}$. However, the effect of alkoxy substitution on oligomerization is significantly smaller than observed for $\mathrm{C}^{*}$---C bond formation and $\mathrm{C}^{*}-\mathrm{OZ}$ activation, with differences ranging from $1-12 \mathrm{~kJ} / \mathrm{mol}$ for oligomerization. This is due to the concerted nature of the mechanism, in which the large cost for $\mathrm{C}^{*}-\mathrm{OZ}$ activation is immediately compensated by the high exothermic gain from simultaneous $C^{*}-C$ bond formation with the reacting alkene. This evidence implies that any effect that further facilitates the $\mathrm{C}^{*}$-OZ activation towards carbenium ion formation will increase activity for all alkoxides towards alkene oligomerization. The effect of alkene size on gas phase oligomerization has an even greater influence on the reactivity, with isobutene oligomerization yielding consistently lower reaction energies than propene oligomerization by the same differences calculated in the $\mathrm{C}^{*---C}$ bond formation step. Therefore, the effect of alkene substitution on oligomerization is the same as that seen with regards to $\mathrm{C}^{*}-\mathrm{C}$ bond formation, in which the stability of the resulting dimerized intermediate dictates the alkene reactivity. These results indicate that the alkene oligomerization activity increases with increasing reactant size due to: (1) stabilization of positive charge on the reacting carbenium, and (2) delocalization of positive charge in the resulting dimerized intermediate. Any properties of the reactants, catalyst, or local environment that stabilize these features would likely increase the catalytic activity for alkene oligomerization. 


\subsection{Alkene Physisorption}

The propene physisorption energy into the zeolite shows only minor changes with changing alkoxide size (Table 3.8.1). This is expected as changes in the alkoxy size would have only weak secondary interactions with the physisorbed propene. In addition, the physisorption energies are comparable across all zeolites tested regardless of zeolite size, with overall energy differences of less than $10 \mathrm{~kJ} / \mathrm{mol}$ as shown in Table 3.8.1. The physisorption in TON is slightly more favorable than the physisorption in MOR and MFI.

Table 3.8.1: Energy of alkene physisorption into zeolite with already adsorbed $\mathrm{C}_{2}-\mathrm{C}_{4}$ alkoxides.

\begin{tabular}{l|l|r|r|r}
\hline Reactant & Alkoxide & TON [kJ/mol] & MFI [kJ/mol] & MOR [kJ/mol] \\
\hline \multirow{4}{*}{ Propene } & Ethoxy & -41 & -45 & -41 \\
& 2-Propoxy & -45 & -40 & -42 \\
& t-butoxy & -49 & -41 & -45 \\
\hline \multirow{5}{*}{ Isobutene } & Ethoxy & -41 & -61 & -52 \\
& 2-Propoxy & -41 & -51 & -52 \\
& t-butoxy & -40 & -54 & -50 \\
\hline
\end{tabular}

The physisorption of isobutene in both MOR and MFI show larger heats of adsorption for isobutene over C2-C4 alkoxides in comparison to propene. The increased size of isobutene increases the number of secondary interactions with both the surrounding zeolite framework, which results in a slight increase in attractive secondary interactions between the adsorbed alkoxy and physisorbed isobutene. Isobutene physisorption in MFI displays varying physisorption values up to $61 \mathrm{~kJ} / \mathrm{mol}$ for isobutene physisorption over ethoxy, but overall remain expectedly higher than those values observed for propene physisorption. However, in the case of TON, the expected increase in physisorption energy is unobserved, with isobutene physisorption remaining at roughly $40 \mathrm{~kJ} / \mathrm{mol}$. Analysis of the isobutene placement (Figure 3.8.2) within TON shows that isobutene is located further down the channel away from the adsorbed alkoxy in comparison to placement of isobutene over the top of the alkoxy as seen in MOR, and isobutene placed at the intersecting sinusoidal channel in MFI. Limitations of the pore size suppress the attractive secondary interactions between isobutene and adsorbed 
alkoxy, and thus hinder the weak increase in heats of adsorption otherwise observed in MFI and MOR.

The loss of entropy due to the secondary interactions of the alkene with surrounding framework results in fairly low free energies of adsorption. This is consistent with the results reported in chapter 2 for aldol condensation. The weakest free energy of adsorption was calculated for propene adsorption into TON at $-4 \mathrm{~kJ} / \mathrm{mol}$, whereas the largest free energy was calculated for isobutene into MOR at $-12 \mathrm{~kJ} / \mathrm{mol}$ (Table 3.8.3). The adsorption of isobutene in TON was the only system calculated to have a positive Gibbs free energy of adsorption. This is likely the result of the bulkiness of the isobutene and zeolite's small size which increases the total number of secondary interactions with the adsorbed isobutene. Even though MFI and TON have similar pore diameters, MFI exhibits more favorable free energies of adsorption in comparison to TON due to its increased chamber diameter from the intersecting perpendicular channels, decreasing the total secondary interactions with the adsorbing alkene and therefore decreasing the overall loss of entropy in comparison to TON.
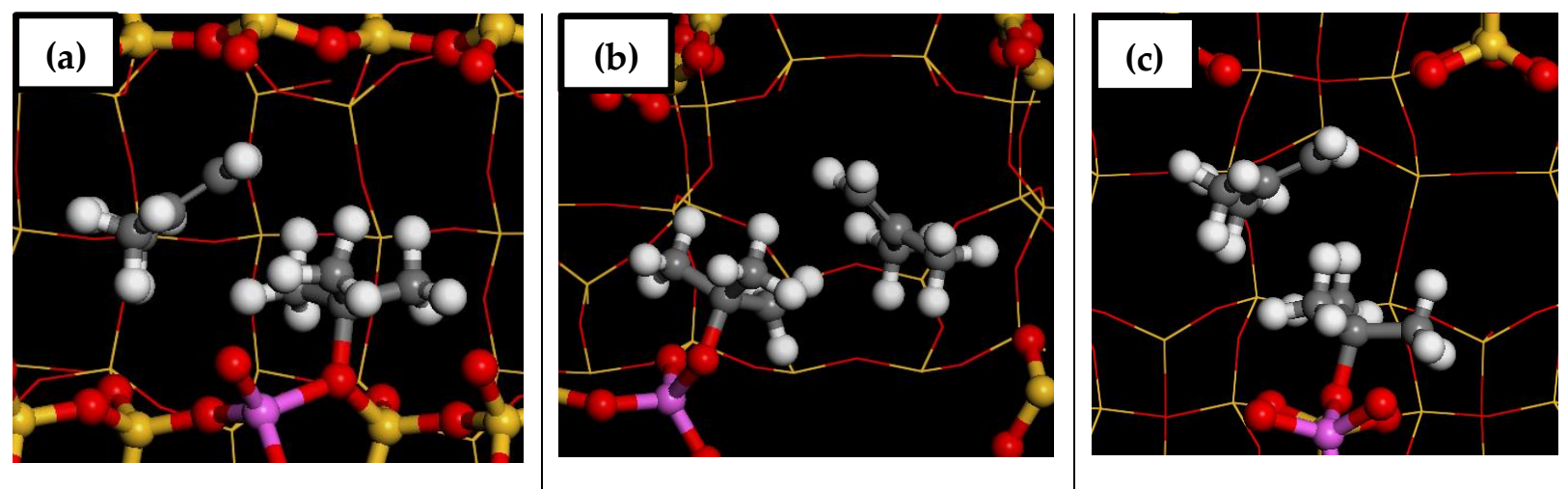

Figure 3.8.2: Isobutene physisorption over adsorbed t-butoxy in (a) TON, (b) MFI, and (c) MOR.

Table 3.8.3: Calculated Gibbs free energy of adsorption of propene and isobutene into TON, MFI, and MOR $(\mathrm{T}=600 \mathrm{~K})$

\begin{tabular}{|l|r|r|}
\hline & $\Delta$ Gads Propene $[\mathrm{kJ} / \mathrm{mol}]$ & $\Delta$ Gads Isobutene $[\mathrm{kJ} / \mathrm{mol}]$ \\
\hline TON & -3.6 & 5.5 \\
\hline MFI & -8.2 & -8.9 \\
\hline MOR & -7.6 & -11.6 \\
\hline
\end{tabular}




\subsection{Intrinsic Activation Energy}

The calculated intrinsic activation energies for propene and isobutene oligomerization monotonically decrease with increasing reactant substitution for all zeolites as shown in Figure 3.9.1. The oligomerization barriers are lowest for TON and highest for MOR with the exception of the reactions of propene-t-butoxy, which is slightly lower in MOR than TON. Increasing the alkoxide substitution has a particularly strong effect on intrinsic activation energy, with the primary carbenium ion center from ethoxy oligomerization consistently yielding higher intrinsic activation barriers than the secondary and tertiary carbenium ion centers from 2propoxy and t-butoxy oligomerization, respectively.

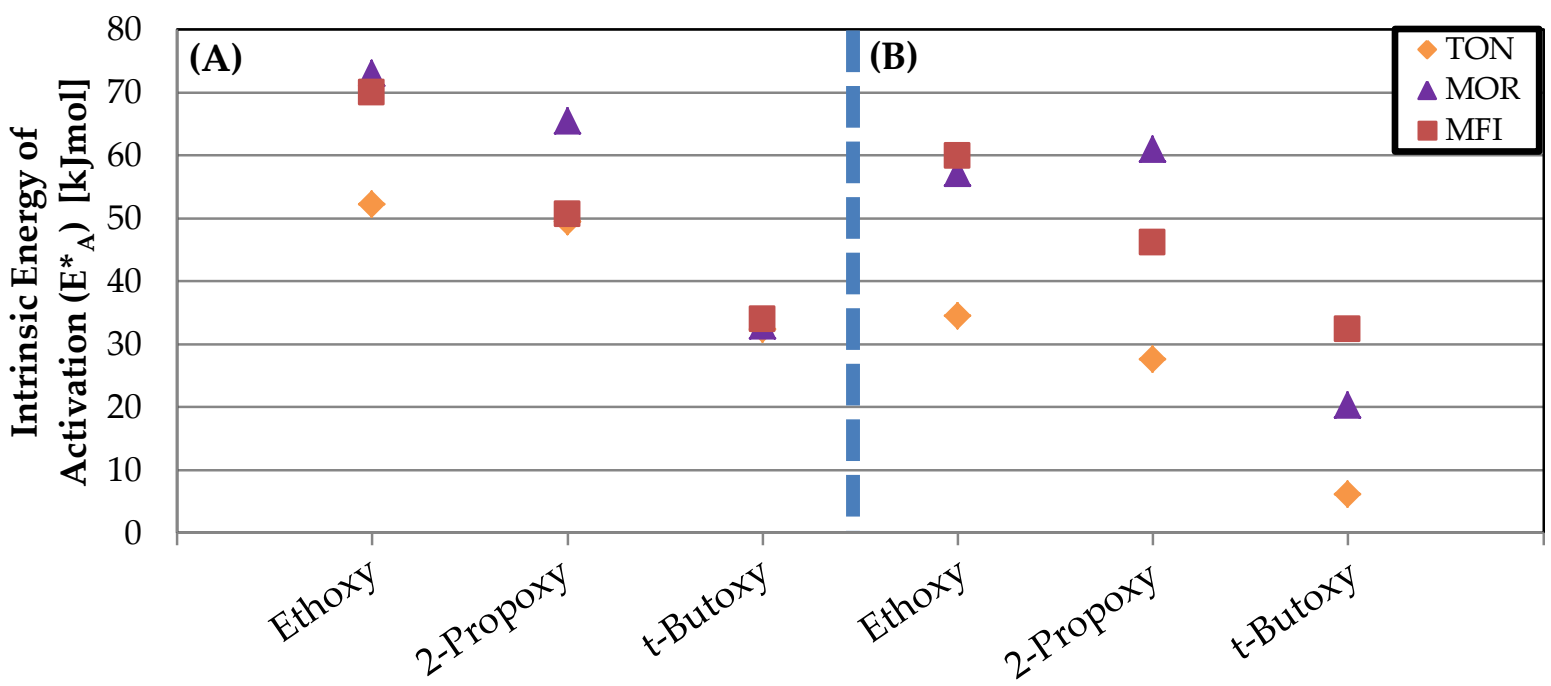

Figure 3.9.1: Intrinsic activation barriers for the reaction of (a) propene and (b) isobutene with ethoxy, 2-propoxy, and t-butoxy in TON, MFI, and MOR zeolites.

The intrinsic $\mathrm{C}-\mathrm{C}$ bond formation barriers in TON decrease with increasing degrees of substitution on the alkene whereby the barriers for isobutene were lower than those for propene for all of the alkoxides examined and is consistent with the gas phase oligomerization energies reported earlier in Table 3.7.4. In TON, however, the difference in the intrinsic barriers for the ethoxy-propene and ethoxy-isobutene oligomerization reactions were calculated to be 15 $\mathrm{kJ} / \mathrm{mol}$, whereas the energy differences for the same gas phase reactions were found to be 50 $\mathrm{kJ} / \mathrm{mol}$. The smaller carrier difference found in TON is largely due to the significantly reduced instability of the solvated carbenium ion within the zeolite framework as compared to that for 
the gas phase carbenium ion. The secondary interactions with the $\mathrm{AlO}^{*}$ site and surrounding zeolite framework help stabilize the positive carbenium ion center throughout the oligomerization reaction, which significantly decreases the energy gain from $C^{*}-C$ bond formation with the physisorbed alkene in a zeolite versus in the gas phase. The cost associated with the activation of the $\mathrm{C}^{*}-\mathrm{O}$ bond to form the carbenium ion is also reduced, but not nearly to the same extent as the solvated carbenium ion center versus a gas phase carbenium ion center in vacuum. The steric limitations due to the repulsion from the larger alkene within the restricted zeolite framework further hinders the energy gain from $C^{*}-C$ bond formation for isobutene versus that of propene and highlights the energetic differences between isobutene and propene oligomerization.

The importance of alkoxy substitution in comparison to alkene substitution is further apparent in comparing the intrinsic activation energies for MOR and MFI systems. The differences that result from changes in the alkoxide from ethoxide to propoxide reduced the barrier by over $30 \mathrm{~kJ} / \mathrm{mol}$ whereas the changes in the alkene from propene to isobutene reduced the barrier by only $10 \mathrm{~kJ} / \mathrm{mol}$. Unlike TON, the intrinsic energy barrier for ethoxy-isobutene is higher than the barrier for t-butoxy-propene oligomerization in both MOR and MFI, which shows the significant impact of substituted alkoxy center versus the substitution of the resulting oligomer carbon center after reaction. The larger MFI and MOR chambers reduce the steric limitations present in TON for isobutene in comparison to propene, but also increase the alkene distance with the alkoxy. The larger chamber size allows isobutene to sit further away from the adsorbed ethoxy or 2-propoxy, reducing the interactions with the carbenium ion center and increasing the $\mathrm{C}^{*}-\mathrm{O}$ activation cost for carbenium ion formation. This also applies to propene for t-butoxy-propene oligomerization, but the tertiary carbenium ion center already has significant stability in comparison to ethoxy and 2-propoxy, and thus the cost for $\mathrm{C}^{*}-\mathrm{O}$ activation does not increase as drastically.

\section{$\underline{3.10 \text { Charge analysis of Alkene Oligomerization in Zeolites }}$}

In an effort to understand the electronic changes that occur along the reaction path for the different reactants, alkoxides and within different zeolites, we carried out a Bader charge 
analysis. We examined the total charge on the alkene, carbenium ion intermediate, and TON zeolite at a series of points taken along the reaction coordinate. Herein we use the normalized $\mathrm{AlO}^{*}-\mathrm{C}^{*}$ bond length from the reactant state to the transition state, and then to the normalized $\mathrm{C}^{*}-\mathrm{C}$ bond length between the carbenium ion carbon center and the reacting alkene carbon from the transition state to the dimerized intermediate state to provide a physical measure of the reaction coordinate. The difference in bond length $(\mathrm{x})$ from the reactant or intermediate resting state is normalized with respect to the difference in bond length between the transition state (TS) and reactant or intermediate state (rest), in which a normalized bond length of 0 signifies the reactant and intermediate states, while a normalized bond length of 1 signifies the transition state, as shown in equation 12.

$$
\text { normalized bond length }=\text { abs }\left[\frac{x-T S}{\text { rest-TS }}\right]
$$

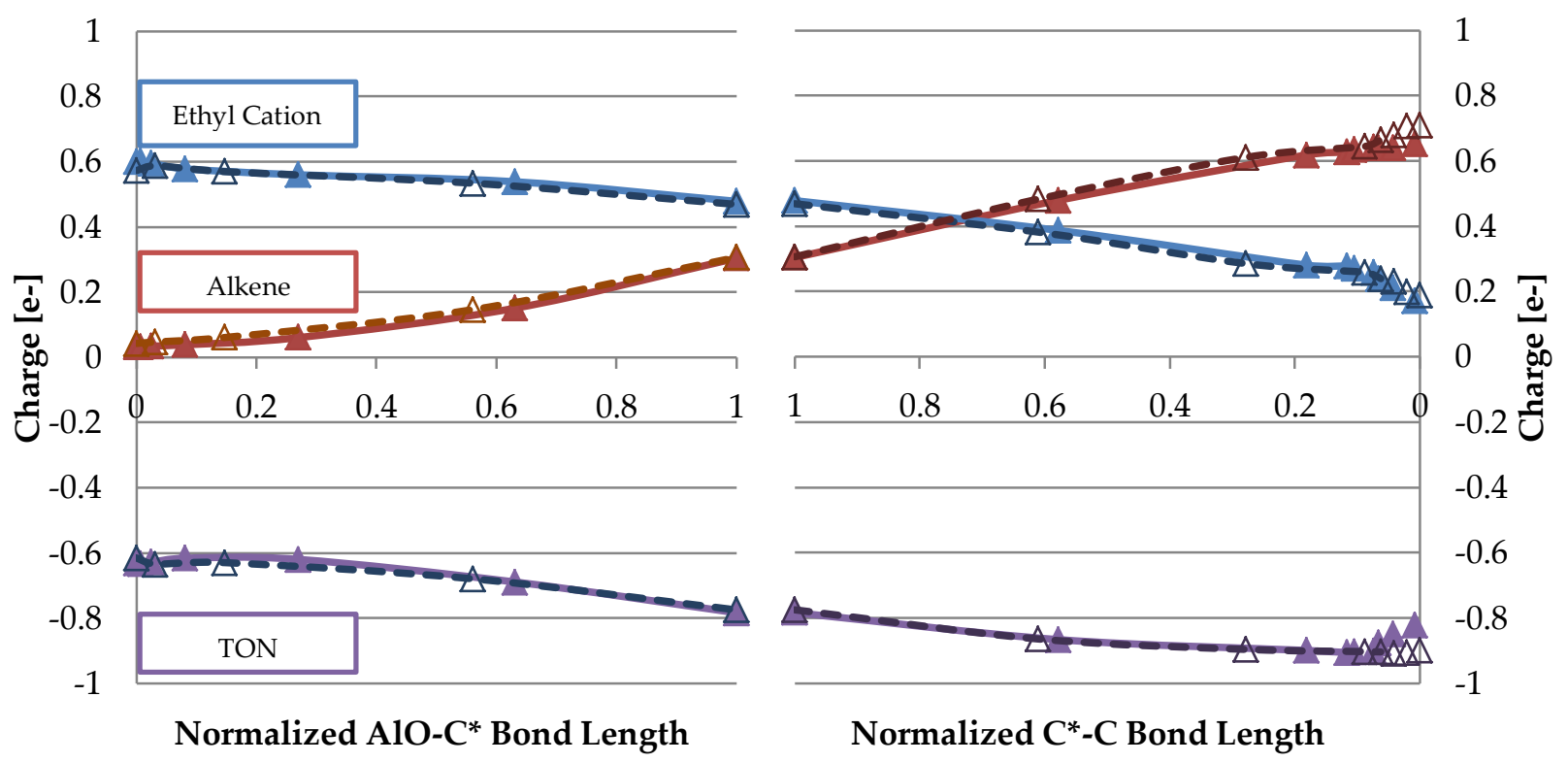

Figure 3.10.1: A Bader charge analysis for the ethoxy-propene $(\mathbf{\Delta})$ and ethoxy-isobutene $(\Delta)$ oligomerization reactions within TON that analyzes the transfer of accumulated charge between the ethyl cation (blue), alkene (red), and surrounding TON framework (purple).

A Bader charge analysis for ethoxy-propene oligomerization reaction in TON reported in Figure 3.10.1 shows that as the reaction proceeds, the $\mathrm{AlO}^{*}-\mathrm{C}^{*}$ bond is activated and there is 
a charge transfer from the resulting carbenium ion complex comprised of the ethyl cation and the ensuing alkene to TON. The negative charge on the TON lattice increases from -0.62 to -0.77 at the transition state. Surprisingly, the ethyl cation carbenium ion intermediate loses a near equal amount of accumulated positive charge as it separates from the negatively charged $\mathrm{AlO}^{*}$ site as it changes from an initial charge of +0.59 to +0.47 at the transition state. This decrease in the positive charge on the ethyl cation along with the increase in negative charge on the zeolite is balanced by an immediate accumulation of positive charge on the alkene. This is consistent with the formation of the $C^{*}-C$ bond between the ethyl carbenium ion center and the $\pi$-bond on the alkene, as well as charge transfer from the olefin to the zeolite lattice even before the initial activation of the $\mathrm{AlO}^{*}-\mathrm{C}^{*}$. This is further evidenced by the minimal charge transfer from the TON zeolite at the beginning of the reaction, in which TON exhibits negligible change in charge until roughly 0.27 along the $\mathrm{AlO}^{*}-\mathrm{C}^{*}$ normalized bond length. This is likely due to the relatively high instability of the small ethyl and its inability to stabilize its positive charge until later along the reaction path where it can form a stronger interaction with the $\pi$-bond of ensuing alkene before the $\mathrm{AlO}^{*}-\mathrm{C}^{*}$ bond is activated.

The charge on the ethyl cation drops significantly from +0.47 at the reactant state to +0.18 after the transition state as it forms the $\mathrm{C}^{*}-\mathrm{C}$ bond and transfers charge to the alkene to form the resulting $\mathrm{C}_{5}$ carbenium ion product. As expected, the ethyl cation still retains some positive charge in the oligomer which is delocalized over the dimerized carbenium ion intermediate. TON continues to accumulate negative charge during $\mathrm{C}^{*}---\mathrm{C}$ bond formation as the charge on TON framework increases from -0.77 charge at the transition state to -0.90 in the dimerized intermediate state. A majority of negative charge accumulates on the TON zeolite before the 0.6 normalized $C^{*}-C$ bond length. This 0.6 normalized $C^{*}-C$ bond length coincides with the change of charge in carbenium ion where the positive charge shifts from the ethyl cation to the reacting alkene and signifies the near-complete rupture of the $\mathrm{AlO}^{*}-\mathrm{C}^{*}$ bond. The TON zeolite continues to increase slightly in negative charge beyond the 0.6 normalized $C^{*}-C$ bond length. This is likely due to a loss of secondary interactions with the ethyl carbenium ion center which becomes more neutral as the dimerized intermediate further separates from the $\mathrm{AlO}^{*}$ site and the positive charge shifts to the reacting alkene. 
(a)

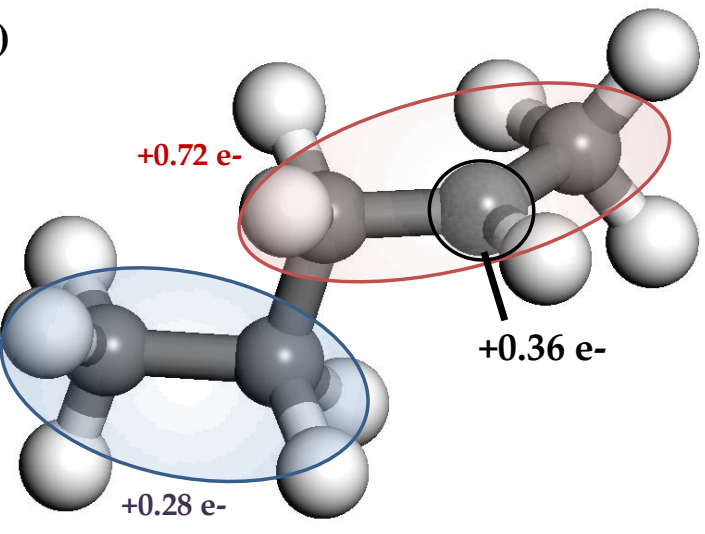

(b)

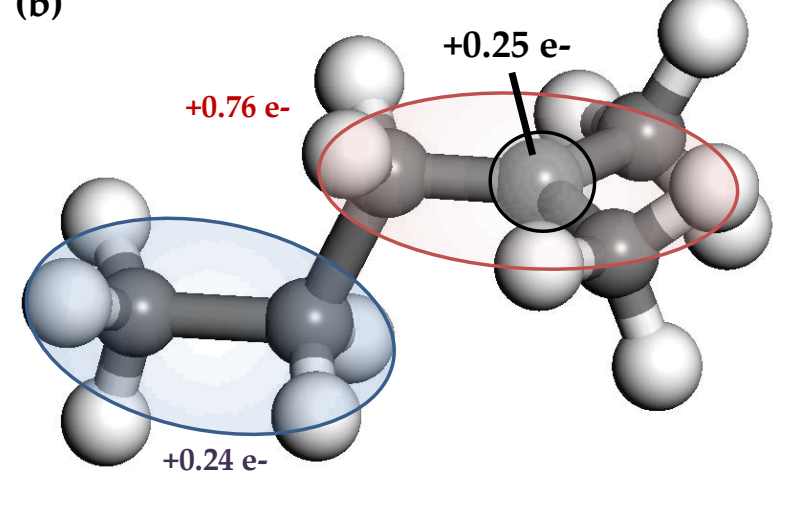

Figure 3.10.2: Mulliken charge analysis of summed charges on ethyl fragment (blue), alkene fragment (red), and oligomer carbenium ion $\mathrm{C}+$ center (black) on the gas phase dimerized intermediates for (a) ethoxy-propene and (b) ethoxy-isobutene reacting pairs.

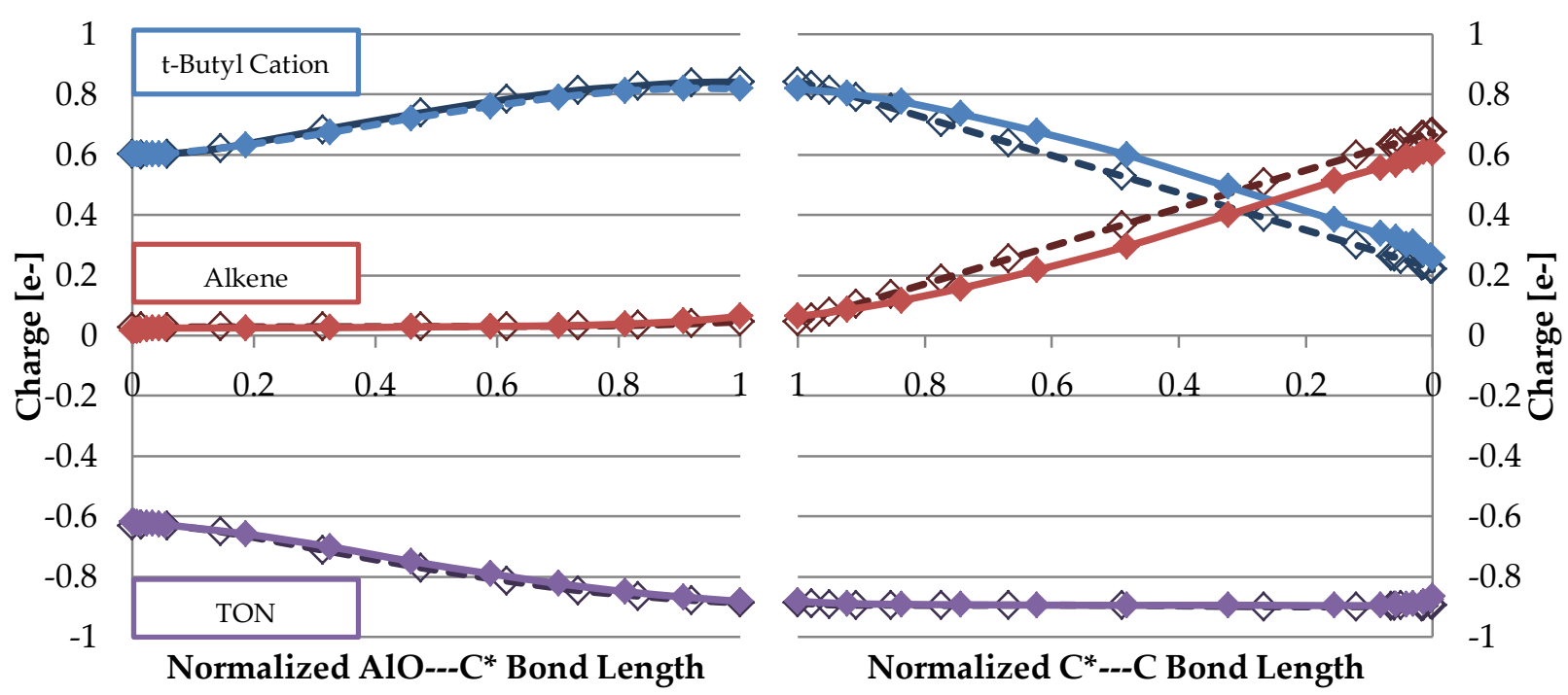

Figure 3.10.3: Bader charge analysis in TON of $\mathrm{t}$-butoxy oligomerization with propene $(\checkmark)$ and isobutene $(\diamond)$, analyzing transfer of accumulated charge between the t-butyl cation (blue), physisorbed alkene (red), and surrounding TON framework (purple).

A similar analysis was carried out to examine the charge transfer that results for $C^{*}-C$ bond forming reaction between isobutene and the bound ethoxide to explore the differences 
associated with changing the degree of substitution of the alkene. The results for the isobuteneethoxide reaction show strikingly little difference to those for the propene-ethoxide reaction as the charge transfer profiles shown in Figure 3.10.1 are nearly identical. The only small difference in these profiles is in the final accumulated charges on the $\mathrm{C}_{5^{-}}$and $\mathrm{C}_{6}$ oligomer intermediate states, where the total charge on the isobutene fragment results in a slightly higher separation of charge $(+0.72)$ than that observed for the charge on the propene fragment $(+0.65)$. A Mulliken charge analysis was carried out using Gaussian calculations to quantify the differences in localized charge on individual atoms. The results shown in Figure 3.10.2 reveal that, while the total ethoxy-isobutene pair accumulates slightly higher positive charge by +0.07 , the resulting unsaturated carbon ion center of the ethoxy-isobutene carbenium ion intermediate $(+0.25)$ has a lower positive charge than that for the ethoxy-propene pair $(+0.36)$. This increased delocalization of positive charge across the full dimerized intermediate illustrates the increased stability of isobutene in comparison to propene and helps to quantify the effect of increased substitution of the alkene for oligomerization.

The results from Bader charge analyses for the oligomerization of propene and isobutene with t-butoxy reported in Figure 3.10.3 also show nearly identical profiles in comparing the two different alkenes. As observed for ethoxy oligomerization, the charge profiles for isobutene-t-butoxy and propene-t-butoxy oligomerization are identical going into the transition state during carbenium ion formation, but show slight differences in charge separation during $C^{*}---C$ bond formation which occurs after the transition state to form the dimerized intermediate as shown in Figure 3.10.3. There is a slightly greater separation of charge in the formation of the isobutene-t-butyl carbenium ion which has charges of +0.22 and +0.66 on the isobutene and t-butyl, respectively, than that for the formation of isobutene-t-butyl carbenium ion which results in charges of +0.28 and +0.61 on the propylene and t-butyl, respectively. 

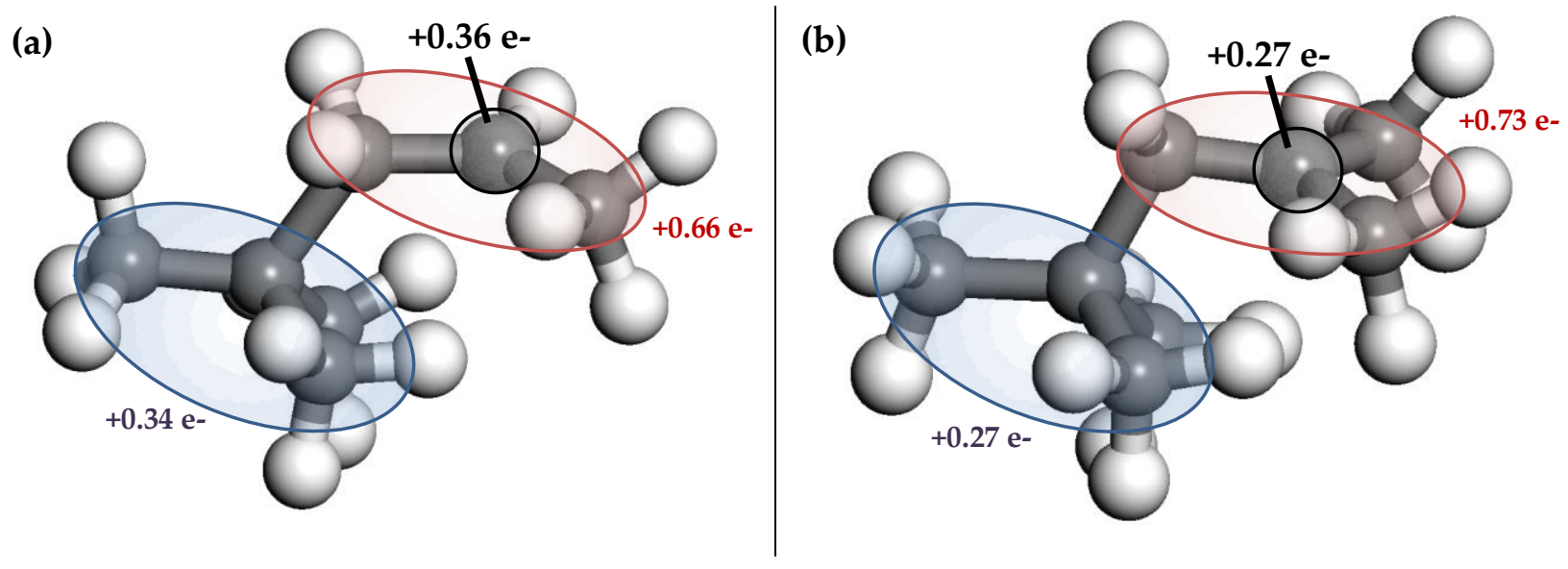

Figure 3.10.4: Mulliken charge analysis reporting the summation of charge on t-butyl fragment (blue), alkene fragment (red), and oligomer carbenium ion $\mathrm{C}+$ center (black) on the gas phase dimerized intermediates for (a) t-butoxy-propene and (b) t-butoxy-isobutene reacting pairs.
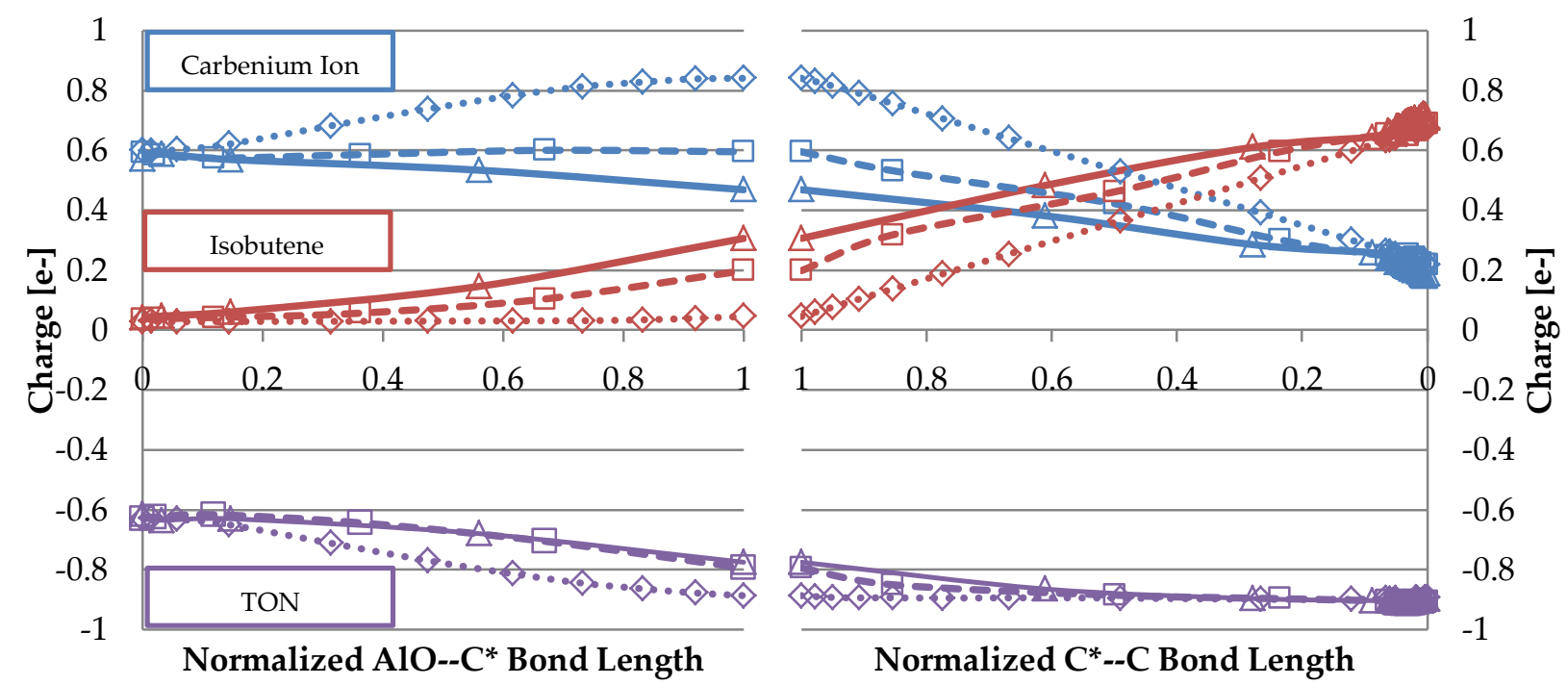

Figure 3.10.5: Bader charge analysis in TON of ethoxy $(\Delta)$, 2-propoxy $(\square)$, and t-butoxy $(\diamond)$ oligomerization with isobutene, analyzing transfer of accumulated charge between the carbenium ion (blue), physisorbed isobutene (red), and surrounding TON zeolite (purple).

The localized charge at the unsaturated carbon on the gas phase oligomer intermediates show a lower accumulation of positive charge on the t-butyl-isobutene pair with Mulliken 
charge of +0.27 verses that on the t-butyl-propene pair which has a Mulliken charge of +0.36 (Figure 3.8.4). This is further evidence that supports the greater delocalization potential of isobutene during oligomerization, and illustrates the effect of increased stability with increased alkene substitution.

The results reported in Figures 3.10.1 and 3.10.3 for the reactions of propene and isobutene with the ethoxy and t-butoxy in TON, respectively, show significant differences for two different alkoxides. In order to elucidate the differences in more detail, we carried out further charge analyses for the reactions of isobutene with ethoxy, 2-propoxy, and t-butoxy to examine the changes between the primary, secondary, and tertiary carbenium ions that result for these systems, respectively, and plot the results in Figure 3.10.5.

The charge analysis for the isobutene reaction with t-butoxy-isobutene in TON reports a higher separation of charge almost immediately following the transition state in comparison to t-butoxy-propene, which displays a delay in charge transfer during $\mathrm{C}^{*}-\mathrm{C}$ bond formation (Figure 3.10.5). A similar effect was also observed with ethoxy, but this difference in separation of charge appears far earlier in the reaction for $t$-butoxy, with $t$-butoxy charge analysis showing differences in charge separation by 0.9 normalized $C^{*}-C$ bond length in comparison to 0.1 normalized $C^{*}-C$ bond length for the ethoxy oligomerization. The differences are the result of the differences in delocalization potential between t-butoxy and ethoxy, rather than differences between the reacting propene or isobutene. In the case of oligomerization with ethoxy, the primary carbon center of the ethyl cation is very unstable, and therefore differences in delocalization between the alkenes are only made apparent near the end of alkene oligomerization where there is a full rupture of the $\mathrm{AlO}^{*}-\mathrm{C}^{*}$ bond. In the case of oligomerization with t-butoxy, the t-butyl cation tertiary carbon center is fairly stable and able to delocalize the accumulated positive charge for longer along the reaction coordinate, thus accentuating differences in delocalization between propene and isobutene earlier in the reaction coordinate.

The effect of alkoxide substitution on alkene oligomerization is made apparent in the large differences in charge along the reaction coordinate for isobutene oligomerization in TON. In both the carbenium ion formation before the transition state and $\mathrm{C}^{*}---\mathrm{C}$ bond formation 
following the transition state, there are significant differences in accumulated positive charge on the carbenium ion intermediates due to the stability of the primary, secondary, and tertiary cations that form from the ethoxide, 2-propoxide, and t-butoxide intermediate. The results show that the ethyl cation accumulates the lowest amount of positive charge whereas the $t$ butoxy accumulates the highest amount of positive charge. As observed previously, the primary ethyl cation immediately interacts with the physisorbed alkene to transfer already accumulated positive charge during $\mathrm{AlO}^{*}-\mathrm{C}^{*}$ bond activation and stabilize the intermediate throughout oligomerization (Figure 3.10.5). In comparison, the secondary 2-propyl cation exhibits minimal charge accumulation during carbenium ion formation going into the transition state, with charge transfer occurring through the carbenium ion and accumulating at the physisorbed alkene and TON zeolite. The tertiary t-butyl cation is the only carbenium ion

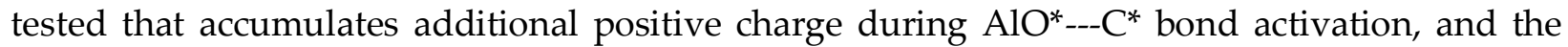
physisorbed alkene does not exhibit any charge accumulation until after the transition state during $C^{*---C}$ bond formation.

(a)

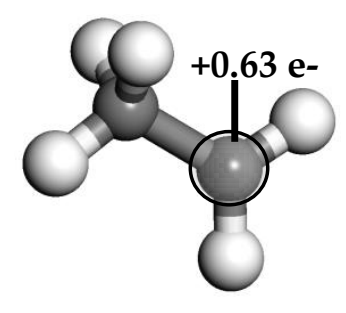

(b)

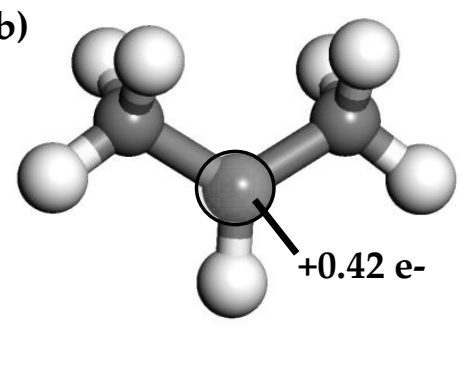

(c)

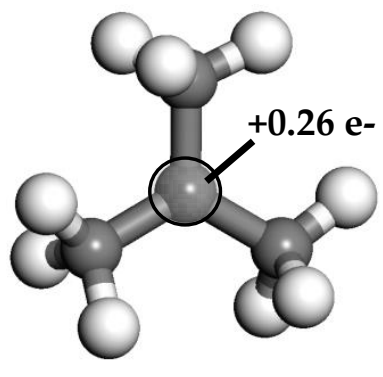

Figure 3.10.6: Mulliken charge analysis of summed charges on carbenium ion C+ center (black) of gas phase (a) ethyl, (b) 2-propyl, and (c) t-butyl carbenium ion intermediates.

A Mulliken charge analysis of the gas phase carbenium ion intermediates quantifies the delocalization potential of the different substituted carbenium ions, with ethyl cation possessing the highest localized positive charge at +0.63 , followed by 2-propyl carbenium ion at +0.42 , and t-butyl carbenium ion illustrating the lowest localized positive charge at +0.26 (Figure 3.10.6). It therefore follows that the observed differences in charge transfer between simulated carbenium 
ions are a direct reflection on the innate stability of the carbenium ion from the degree of substitution at the positive carbon center.
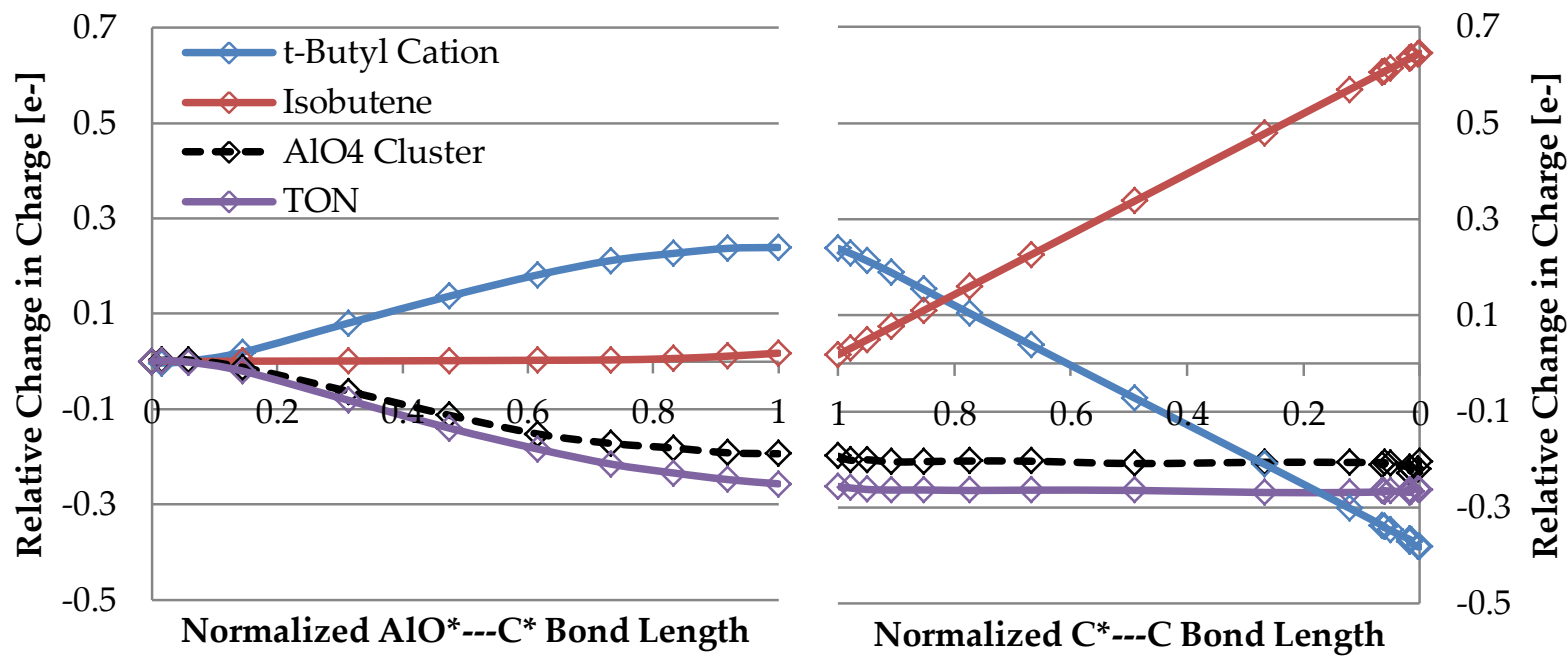

Figure 3.10.7: A Bader charge analysis for t-butoxy oligomerization with isobutene in TON analyzing total change in accumulated charge between the t-butyl cation (blue), physisorbed isobutene (red), $\mathrm{AlO}_{4}$ catalytic cluster within $\mathrm{TON}$ (black), and the sum of charge across the total TON zeolite (purple).

To better understand the nature of $\mathrm{C}^{*---O}$ bond cleavage, a comparison of the relative change in charge for t-butyl-isobutene oligomerization in TON was analyzed. Figure 3.10.7 shows equal charge transfer between the t-butyl cation and TON zeolite, the majority of which is localized at the $\mathrm{AlO}_{4}$ catalytic cluster. There is only -0.06 distributed throughout the remaining $\mathrm{SiO}_{2}$ framework. This localized separation of charge during carbenium ion formation is indicative of heterolytic $\mathrm{C}^{*}$---O bond cleavage, where the majority of charge transfer occurs between the two separating species while the surrounding zeolite and physisorbed alkene seemingly act as spectator molecules. The t-butyl charge analysis suggests that ethoxy and 2-propoxy also undergo heterolytic $C^{*}$---O cleavage, but their associated charge profiles do not illustrate the expected isolated separation of charge as both the $\mathrm{C}^{*}$---O bond cleavage and $\mathrm{C}^{*}---\mathrm{C}$ bond formation steps happen almost simultaneously due to the instability inherent to the primary and secondary carbon centers. 

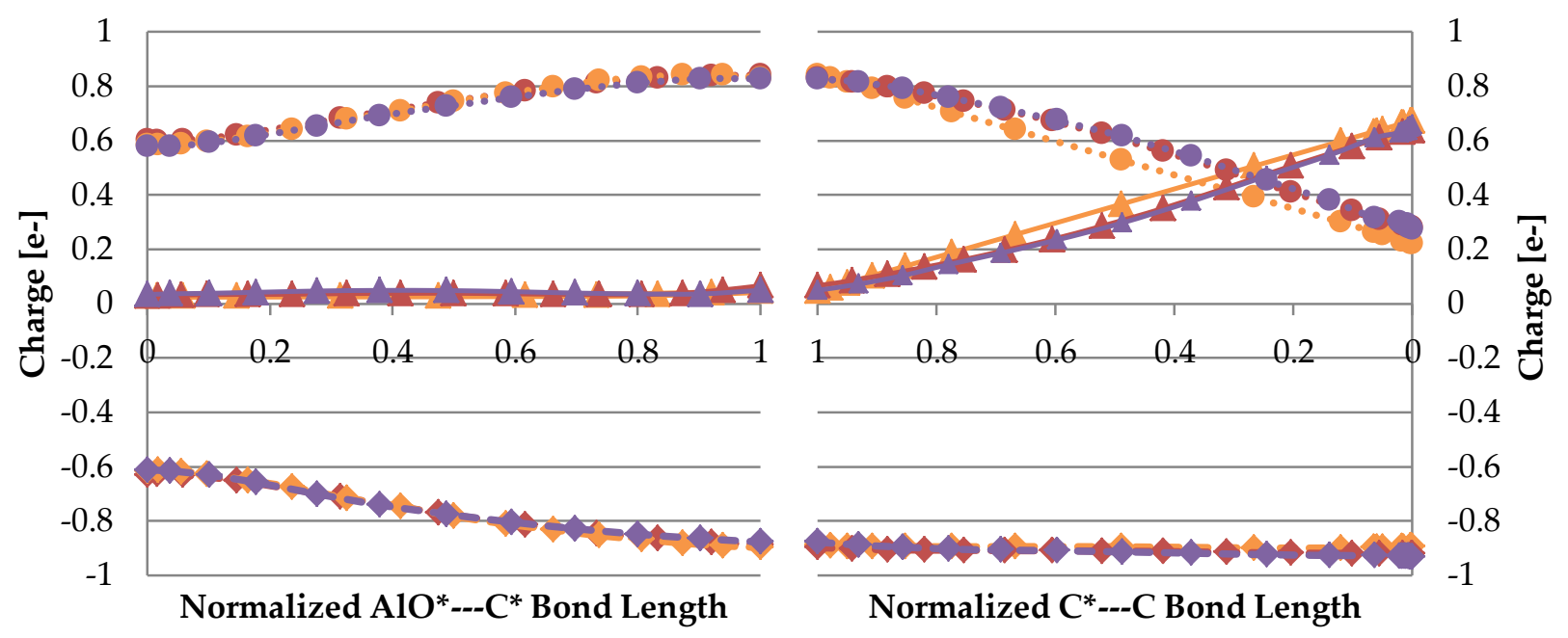

Figure 3.10.8: Bader charge analysis of t-butoxy oligomerization with isobutene in TON (orange), MFI (red), and MOR (purple), analyzing total change in accumulated charge between the t-butyl cation $(\bullet)$, physisorbed isobutene $(\mathbf{\Delta})$, and the sum of charge across the zeolite $(\bullet)$.

A detailed comparison of charge profiles for t-butyl-isobutene oligomerization in TON, MFI, and MOR were carried out to examine the influence of the zeolite on the charge transfer. The resulting charge profiles for TON, MFI, and MOR shown in Figure 3.10 .8 are nearly identical. It is expected that the zeolites yield similar charge magnitudes at the reactant state and transition state as they differ primarily in geometric structure, but not in $\mathrm{AlO}^{*}$ catalytic site strength or $\mathrm{SiO}_{2}$ framework chemical composition. However, the direct overlap of the charge profiles for the entire reaction coordinate implies that the reacting intermediates have no significant coulombic differences at any point during carbenium ion formation. Inspection of the exact $\mathrm{AlO}^{*}-\mathrm{C}^{*}$ and $\mathrm{C}^{*}-\mathrm{C}$ bond lengths at the transition state show that TON, MFI, and MOR bond lengths differ by less than $0.1 \AA$, further supporting the irrelevance of zeolite structure on coulombic interactions.

The only observed difference for alkene oligomerization is during $C^{*}-C$ bond formation, where TON shows charge transfer between isobutene and t-butyl cation sooner along the normalized $C^{*}-C$ reaction coordinate. This is due to the $C^{*}-C$ bond length at the transition state being smaller in TON than for MOR and MFI, which allows the transfer of positive charge 
sooner along the reaction coordinate in TON due to the already close proximity of the reacting t-butyl cation and isobutene. However, these slight differences in charge transfer are not enough to account for the $20-30 \mathrm{~kJ} / \mathrm{mol}$ differences in activation energy between zeolites, and it is therefore apparent that coulombic differences are not the main driving force behind differences in catalytic activity of certain zeolites for alkene oligomerization.

\section{$\underline{3.11 \text { Close-contact Analysis and Van der Waals Volume Overlap }}$}

In order to assess the weak attractive van der Waal interactions between the transition state structures and the zeolite cavity that stabilize the reaction transition state, we calculate the van der Waal volume overlap and the number of close contacts for the oligomerization transition states. Close contact bonds are defined herein as the bonds between the carbenium ion transition state structure and those of the zeolite that are shorter than the van der Waal bond lengths.

Table 3.11.1: Close-contact bond and vdW volume overlap measurements between isobutene transition states and surrounding zeolite framework.

\begin{tabular}{|c|c|c|c|c|c|c|}
\hline & Alkoxy & \# Bonds & $\begin{array}{c}\text { Total vdW } \\
\left.\text { Overlap [ } \AA^{3}\right]\end{array}$ & $\begin{array}{l}\text { Alkene vdW } \\
\text { Overlap }\left[\AA^{3}\right]\end{array}$ & $\begin{array}{l}\text { Alkoxy vdW } \\
\text { Overlap }\left[\AA^{3}\right]\end{array}$ & $\begin{array}{c}\text { \% Alkoxy vdW } \\
\text { Overlap }\end{array}$ \\
\hline \multirow{3}{*}{ TON } & Ethoxy & 41 & 9.1 & 2.4 & 6.7 & $74 \%$ \\
\hline & 2-Propoxy & 47 & 9.8 & 2.7 & 7.1 & $72 \%$ \\
\hline & t-Butoxy & 82 & 19.7 & 7.9 & 11.8 & $60 \%$ \\
\hline \multirow{3}{*}{ MFI } & Ethoxy & 21 & 7.5 & 0.8 & 6.6 & $89 \%$ \\
\hline & 2-Propoxy & 29 & 7.7 & 1.9 & 5.8 & $76 \%$ \\
\hline & t-Butoxy & 41 & 9.9 & 7.1 & 2.8 & $28 \%$ \\
\hline \multirow{3}{*}{ MOR } & Ethoxy & 15 & 5.2 & $<0.1$ & 5.1 & $99 \%$ \\
\hline & 2-Propoxy & 26 & 4.3 & 0.5 & 3.8 & $88 \%$ \\
\hline & t-Butoxy & 24 & 1.8 & 0.3 & 1.8 & $86 \%$ \\
\hline
\end{tabular}

The number of close-contact bonds and van der Waals (vdW) volume overlap between transition state structures and the surrounding zeolite framework were used together with the DFT optimized transition state structures (Table 3.11.1) to quantitatively estimate the difference in secondary interactions between the transition states and the different zeolites. The vdW 
volume overlap was calculated as the volume of overlapping spheres whose radii equal to the $\mathrm{vdW}$ radius of each element. The vdW radius was set as the limit for observed secondary interaction as molecules lying outside of this radius are hypothesized to have minimal effects on the adsorbed transition state molecule within the zeolite framework.

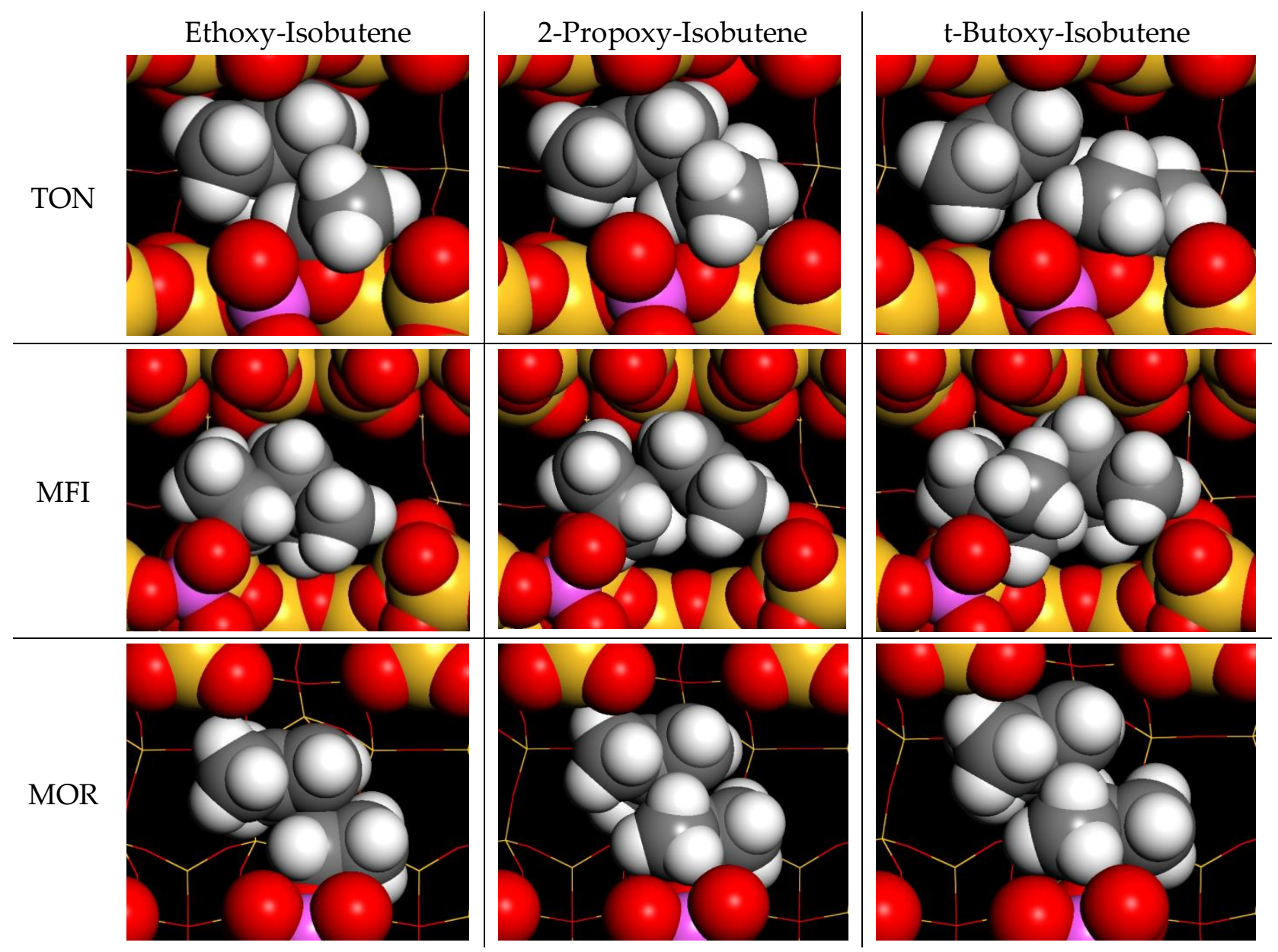

Figure 3.11.2: Illustration of $\mathrm{vdW}$ sphere models of isobutene oligomerization transition states with ethoxy, 2-propoxy, and t-butoxy within the TON, MFI, and MOR.

A comparison of the number of close-contact bonds between isobutene oligomerization transition states reported in Table 3.11.1 show distinct differences in the number of secondary interactions, with TON showing the highest number of potential secondary interactions, followed by MFI and MOR. The high number of close-contact bonds emphasizes the increasing number of potential weak secondary interactions in TON that help to further stabilize the 
transition state structure during reaction, and provides insight into the lower activation energies in TON in comparison to MOR and MFI, despite having the same $\mathrm{AlO}^{*}$-type site and near identical charge transfer potential across the oligomerization reaction. While the number of close-contact bonds alone cannot distinguish between attractive or repulsive interactions, it is clear that the TON 1-D structure shows a higher potential of stability for the transition state than otherwise noted for the larger MOR 1D structure and for the intersecting chamber in MFI.

The calculation of overlapping vdW spheres estimates an expectedly high degree of overlap between the isobutene oligomerization transition state and surrounding zeolite in TON. Larger transition states yielded higher vdW volume overlap with the surrounding framework, with the t-butoxy-isobutene oligomerization transition state in TON yielding nearly double the $\mathrm{vdW}$ overlap volume that was calculated for 2-propoxy-isobutene transition state. The high amount of calculated vdW interaction is indicative of the increased stabilization potential of the smaller TON structure, and helps to explain the lower activation energies for alkene oligomerization.

Both MFI and MOR yielded lower vdW overlap than TON. The intersecting perpendicular channels in MFI allow for additional space which decreases possible secondary interactions. The 8-member alternating side pockets as well as larger pore diameter in MOR greatly decrease secondary interactions with the isobutene oligomerization transition states. In particular, MOR was found to yield decreasing vdW volume overlap with increasing alkoxy size and less than $0.5 \AA^{3}$ overlap between the isobutene and the MOR channel "ceiling". From the Bader charge analysis, the alkene molecule is shown to remain fairly neutral going into the oligomerization transition state, and thus too much alkene interaction with the zeolite ceiling will likely be repulsive rather than attractive. This is a reflection on the alkoxy stability, as the higher substituted carbenium ions are able to sit further away from the $\mathrm{AlO}^{*}$ site due to inherent stability, while the 8-member side pocket allows the transition state to sit relatively center to the MOR chamber as shown in the computational models (Figure 3.11.2).

A comparison of vdW volume overlap between the alkene and the zeolite "ceiling" versus the alkoxy and the surrounding framework reveals that the reacting alkoxy makes up the majority of total calculated vdW overlap with the transition state complex. The Bader 
charge analyses of the carbenium ions reported in section 3.10 indicate that the alkoxy gains positive charge during $\mathrm{C}^{*}$---O activation. Therefore the secondary interactions between the alkoxy and zeolite likely assist in stabilizing the transition state, and the transition state remains close to the $\mathrm{AlO}^{*}$ in order to maximize this effect depending on the inherent stability of the reacting alkoxy. However, previous Bader charge analysis shows the reacting isobutene to be either fairly neutral or slightly positive going into the transition state, and therefore large vdW volume overlap between the isobutene and the zeolite "ceiling" is likely indicative of steric limitations of the zeolite, rather than increased stabilization to the transition state. C8 transition states in TON and MFI showed particularly high vdW volume overlap for the alkene, and this suggests possible steric limitations of the zeolite framework on large transition states.

\subsection{Apparent Activation Energy and Heats of Reaction}

The intrinsic activation barriers presented in section 3.9 which are taken with respect to a bound alkoxide and a gas phase alkene provide important insights in the energetic costs in breaking the $\mathrm{C}-\mathrm{O}$ bond of the alkoxide and forming the $\mathrm{C}-\mathrm{C}$ bond with the free alkene. The apparent activation barriers that are determined experimentally at higher alkene pressures, however, are measured with respect to the bound alkoxide and a physisorbed alkene [14]. The apparent barriers are readily calculated by adding the intrinsic activation energies with the heat of physisorption for the alkene. The plot of the apparent energies of activation for propene oligomerization with different alkoxides in TON shown in Figure 3.12.1 shows an increasing favorability with increasing reactant size and degree of substitution, with ethoxy-propene oligomerization yielding the highest apparent barrier and t-butoxy-propene oligomerization yielding the lowest barrier for all of the tested alkoxides. This is the same ordering found for the intrinsic barriers where we showed that the more substituted alkoxides better delocalize the positive charge for the reacting alkoxy's carbenium ion center. 


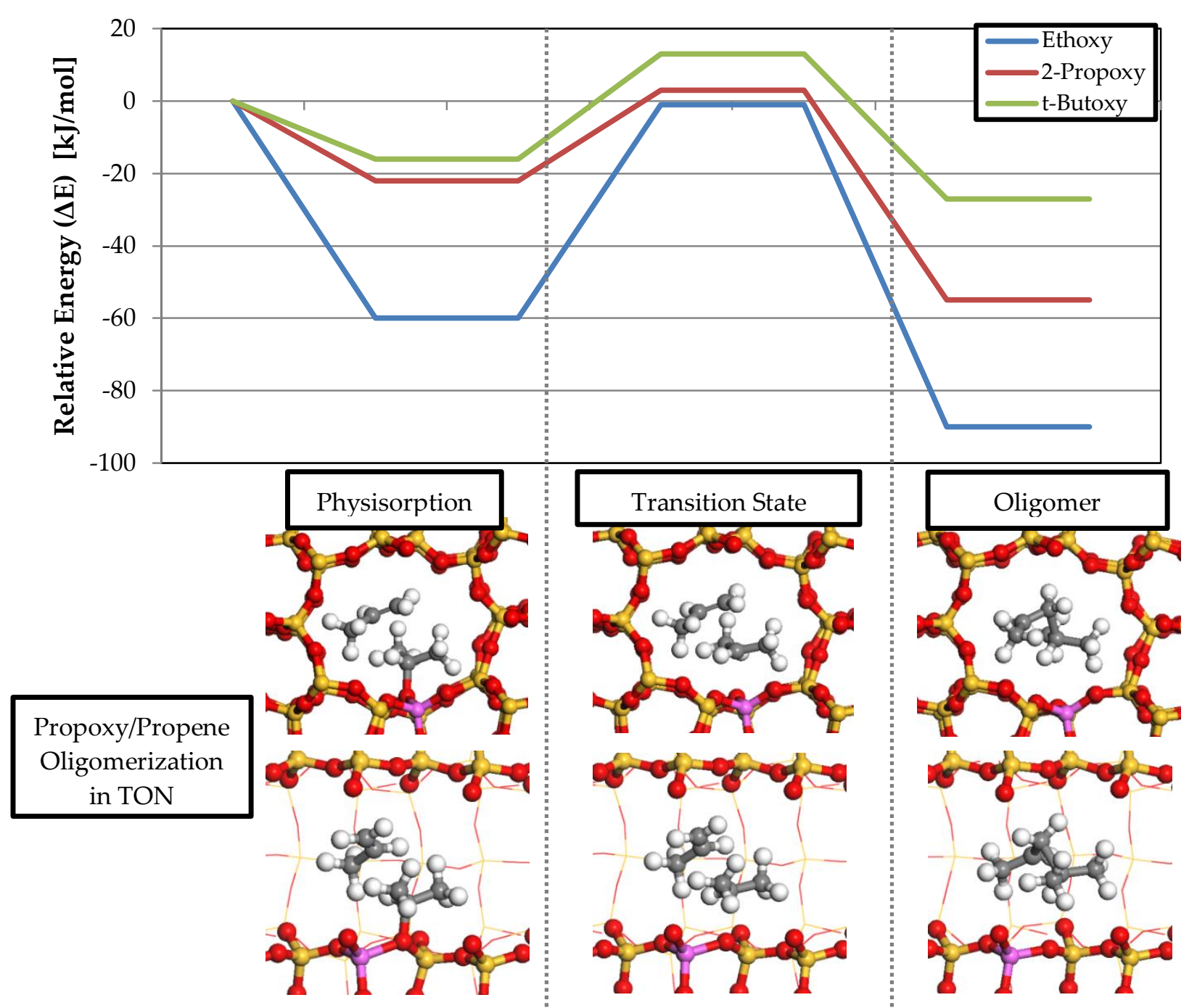

Figure 3.12.1: Apparent energy of activation for alkene oligomerization in TON for propene coupling with ethoxy, 2-propoxy, and t-butoxy.

A comparison of the barriers for all of the alkene oligomerization reacting pairs tested across TON, MFI, and MOR further illustrates the effect of increasing substitution with decreasing apparent energies of activation, as was observed previously in Figure 3.12.2 analyzing intrinsic activation energies. The increased heats of adsorption of isobutene in comparison to propene, however, act to significantly lower the apparent activation barriers and display a stronger influence of the alkene on the resulting apparent activation energies. As such, the effect of the alkene on the apparent barrier appears to be more greatly influenced by the difference in the heats of adsorption into the zeolite rather than solely on secondary 
interactions with the alkoxy and the delocalizing potential of the resulting dimerized intermediate's positive carbon center. This influence of alkene physisorption has also been noted in literature. Ying et al., for example, analyzed the kinetics of $\mathrm{C}_{2}$ - $\mathrm{C}_{7}$ olefin interconversion for alkene oligomerization, aromatization, and cracking reactions at moderate temperatures and low pressure in ZSM-5 [28] and reported negative apparent energies for propene oligomerization and oligomerization-cracking reactions due to the strong physisorption energies which exceeded the intrinsic surface activation energies.

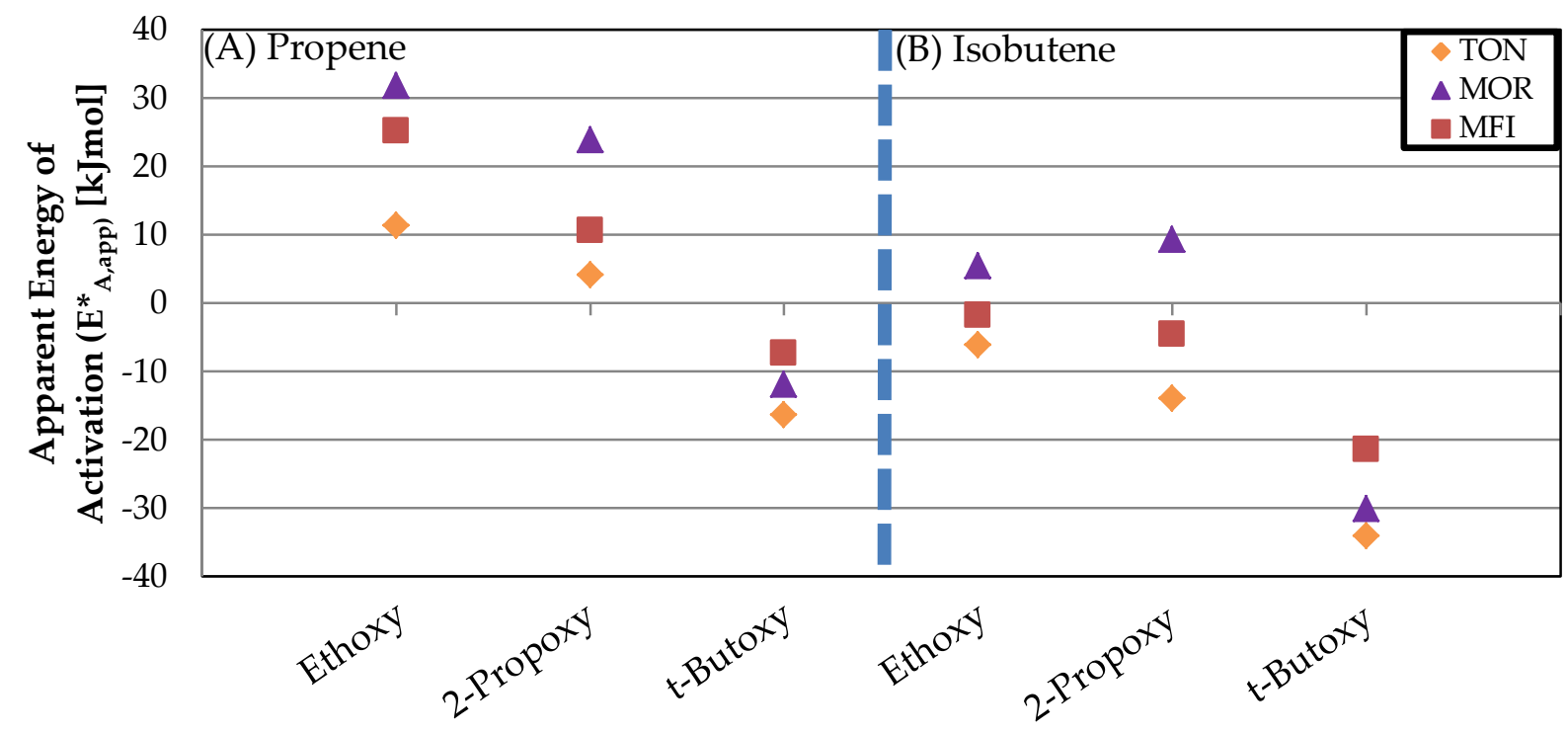

Figure 3.12.2: Apparent energy of activation for (A) propene oligomerization and (B) isobutene oligomerization with ethoxy, 2-propoxy, and t-butoxy in TON, MFI, and MOR.

The apparent activation energies for TON are consistently lower than those for MFI and MOR, while those for MOR are the highest with the one exception being the t-butoxy-propene oligomerization. The apparent energies of activation decrease with increasing reactant size for all zeolites, with the exception of isobutene-2-propoxy oligomerization in MOR. MOR and MFI are shown to benefit more from the adsorption energy of propene and isobutene for the larger reactant pairs than otherwise observed for TON. The close-contact analysis illustrated this effect by showing increasing reactant interaction with increasing reactant size for MFI and MOR. However, in the case of TON, the smaller zeolite size shows limitations with regards to 
reactants within the zeolite, where some of the interactions are repulsive. This is particularly evident in oligomerization with isobutene, as the larger MFI and MOR zeolites are better able to accommodate the bulkier alkene in comparison to TON, and thus the difference in apparent activation energies becomes smaller than otherwise shown in the intrinsic activation energies discussed previously.

\subsection{Apparent Gibbs Free Energy of Activation}

The apparent Gibbs free energies of activation were calculated using vibrational frequency calculations and taken with respect to the adsorbed alkoxy and gas phase alkene. The apparent free energies for isobutene adsorption were calculated to be higher than those for propene due to the greater loss of entropy associated with isobutene adsorption into the zeolite as observed previously for Gibbs free energy of adsorption calculations (Table 3.8.1). Additional entropy is lost after adsorption at the transition state due to the interaction between the alkene $\pi$-bond and the carbenium ion at the transition state. Due to the early nature of the transition state discussed during Bader charge analysis in section 3.10, the carbenium ion carbon center does not interact strongly with the physisorbed alkene through a formal $\mathrm{C}^{*--C}$ bond, but instead through weak interactions across the alkene $\pi$-bond as shown in Figure 3.13.1 with $C^{*}--C$ bond lengths of over $2 \AA$ between the carbenium ion center and alkene $\pi$-bond carbons for all oligomerization transition state pairs, in comparison to the typical $1.6 \AA \mathrm{C}^{*--C}$ bond length found at the dimerized intermediate state. These weak interactions with the carbenium ion coupled with the additional secondary interactions with the surrounding zeolite framework restrict the translational and rotational freedom of the alkene with respect to the gas phase. Therefore, it is expected that the overall reaction will yield a significant loss of entropy and result in free energies of activation larger than the enthalpic activation energies. 


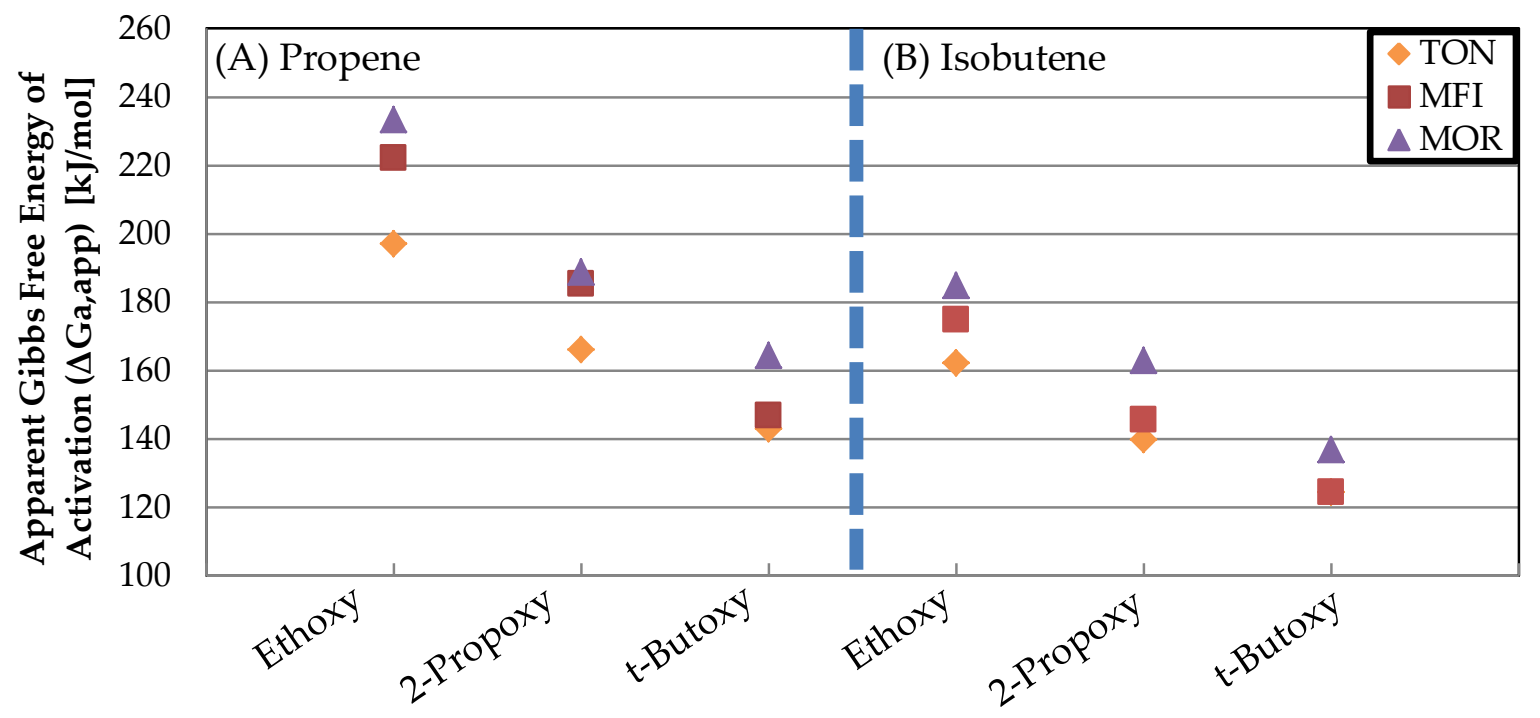

Figure 3.13.1: Apparent Gibbs free energy of activation for C2-C4 alkoxides in TON, MFI, and MOR for (a) propene oligomerization and (b) isobutene oligomerization ( $\mathrm{T}=600 \mathrm{~K})$.

A comparison of entropy gains for propene oligomerization over that of isobutene oligomerization reveals that smaller reactant pairs yield greater losses in entropy, likely due to the lower delocalization over the reacting intermediates. The high instability of smaller carbenium ion intermediates would increase the strength of interaction between the carbenium ion and the physisorbed alkene, and further restrict on the vibrational, translational, and rotational freedom of the alkene at the transition state with respect to the gas phase alkene. For all zeolites, oligomerization with ethoxy yielded the greatest loss in entropy while t-butoxy yielded the smallest loss of entropy, even though the energy for $\mathrm{C}^{*}$--O activation is the lowest of the alkoxides tested. As vibrational contribution to entropy is influenced by how rigid the carbenium ion becomes in the transition state, differences in the $\mathrm{C}^{*}$--O bond strength affect the overall entropy change. Thus, the stability of the carbenium ion at the transition state is the main difference resulting in changes to entropy with regards to alkoxy substitution. A similar dependence was also observed for the alkenes, as propene oligomerization typically yielded higher losses in entropy than those observed for isobutene oligomerization, with similar reasoning associated with these observed differences. 
Even with entropy considered and taken with respect to the gas phase alkene, the Gibbs free energies of activation emphasize the importance of substitution for both the alkene and alkoxy reacting species, with larger reactants being more reactive for alkene oligomerization for all tested zeolites (Figure 3.13.1). A comparison of the free energies of reactions taken across all of the zeolites examined indicates that TON is the most active for alkene oligomerization. This is expected, as TON is shown to have the largest number of close-contact interactions with the adsorbed intermediates, and it should therefore follow that TON would exhibit the highest loss of entropy with respect to those observed for the larger MFI and MOR zeolites due to greater restriction on vibrational freedom of the reacting intermediates. While TON does show comparable entropies, the differences in entropy between TON, MFI, and MOR are roughly 10 $\mathrm{kJ} / \mathrm{mol}$, which is not enough to counteract the stabilizing effect the smaller TON zeolite framework has upon the transition state. Of the zeolites studied, MOR shows the smallest change in entropy due to the relatively greater vibrational and rotational freedom in the larger MOR chamber, and yields apparent Gibbs free energies of activation comparable to those calculated for MFI for larger reactant pairs.

\subsection{Born Haber Cycle Analysis and Interaction Energy}

A Born Haber cycle analysis was used to decompose the alkene oligomerization reaction into a sequence of fundamental thermochemical steps that make up the changes that occur in the reactant and the catalyst in taking the initial reactant and catalyst state to the transition state as shown in Figure 3.14.1. These steps provide direct insights into the critical steps that control the free energy of activation. Herein we start with the bound alkoxy state ZO-R 1 . The first step in the cycle involves the complete "dealkylation" of the zeolite-bound alkoxide to form the gas phase carbenium ion $\mathrm{R}_{1}(+)$ and the negatively charged zeolite, $\mathrm{ZO}(-)$. The energy for this

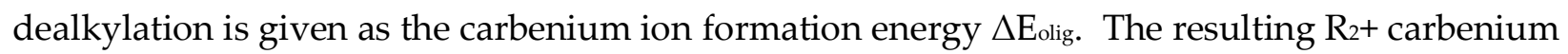
ion intermediate is then rebound to the zeolite to give the corresponding transition state complex to yield the interaction energy $\left(\Delta \mathrm{E}_{\text {int }}\right)$ between the transition state and the zeolite. The intrinsic activation barrier can then be written as the sum of the energies associated with each of the thermochemical steps. 


$$
\Delta E_{A, i n t}=\Delta E_{C F E}+\Delta E_{\text {olig }}+\Delta E_{\text {int }}
$$

The apparent barrier is simply the intrinsic barrier plus the heat of physisorption of the alkene.

$$
\Delta E_{A, \text { app }}=\Delta E_{\text {ads, alkene }}+\left(\Delta E_{C F E}+\Delta E_{\text {olig }}+\Delta E_{\text {int }}\right)
$$

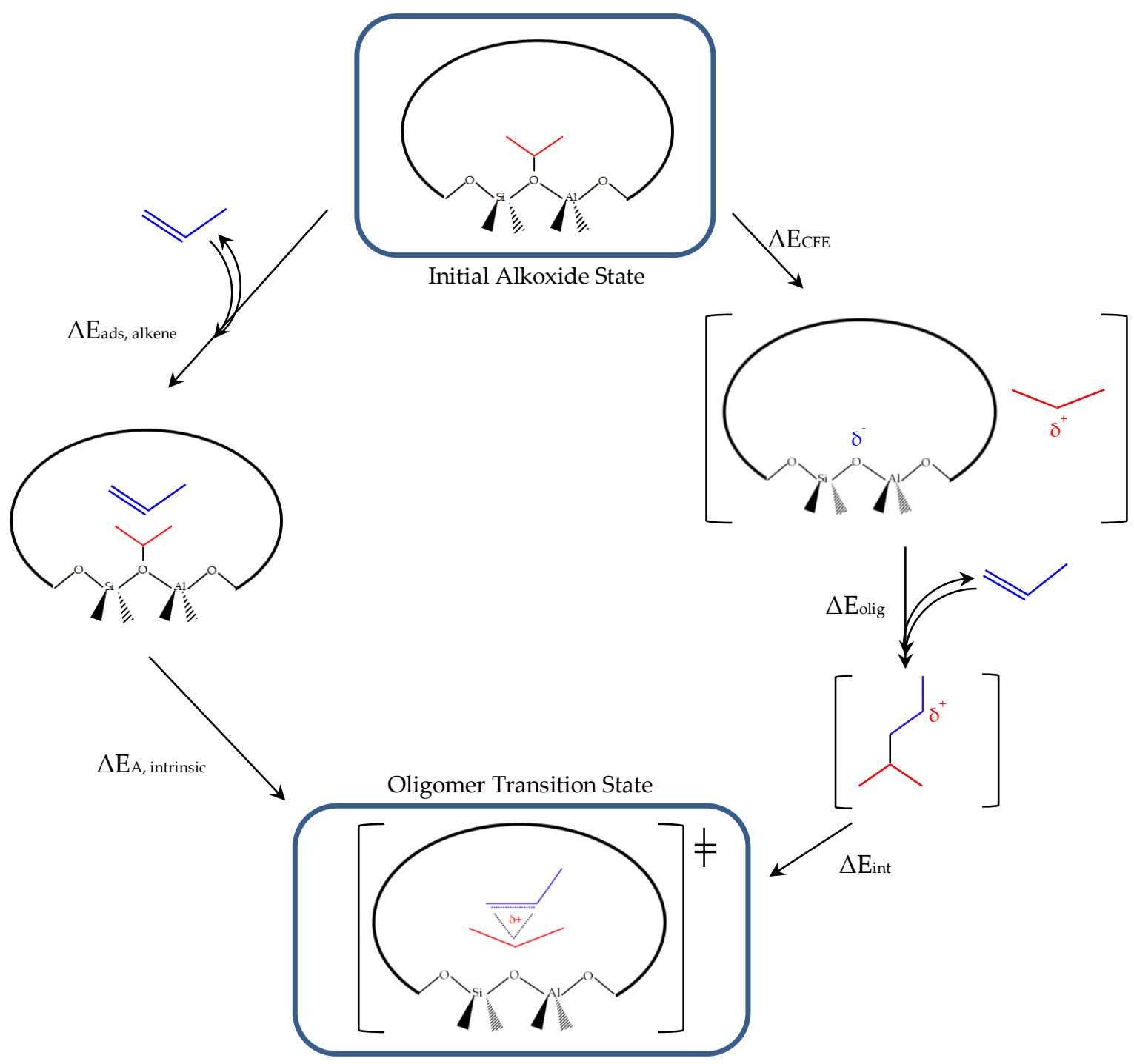

Figure 3.14.1: Born-Haber cycle for alkene oligomerization starting from adsorbed alkoxide in zeolite and ending at dimerized carbenium ion intermediate. Left side energies consist of alkene physisorption into zeolite (Eads, alkene) and intrinsic energy of activation for alkene oligomerization ( $\mathrm{E}_{\mathrm{A}}$, intrinsic). Right side energies consist of carbenium ion formation (EcF), gas phase oligomerization (Eolig), and interaction energy between the oligomer and zeolite framework (Eint). 
Herein we explore in detail the influence of both the carbenium ion formation energy as well as the interaction energy on the calculated activation barriers as they provide quantitative measures of how the intermediates bind to the zeolite. It should be noted that these steps are very similar in that they provide a measure of the binding of a carbenium ion center to zeolite, but are opposite in their sign as $\Delta \mathrm{E}_{\mathrm{CFE}}$ involves the removal of the $\mathrm{R}_{1}(+)$ carbenium ion. As gas phase $R_{2}(+)$ species is more stable than $R_{1}(+)$, the interaction energy is always smaller than the carbenium ion formation energy.

The apparent free energies reported in section 3.13 were taken with respect to the adsorbed alkoxy state and gas phase alkene. Therefore, there are two direct energetic influences that would lower the overall apparent energy: a less stable reactant state, or a more stable transition state. A less stable adsorbed alkoxy as described by carbenium ion formation energy $\left(\Delta \mathrm{E}_{\mathrm{CFE}}\right)$ would increase the energy of the reactant state, and therefore lower the overall apparent barrier.

$$
\Delta E_{C F E}=\left(E_{\text {alkoxy,gas }}+E_{\text {zeolite,blank }}\right)-E_{\text {zeoilte }+ \text { alkoxy }}
$$

In addition, influence of the zeolite at the transition state would directly affect the height of the transition state barrier, and influence the overall apparent barrier.

$$
\Delta E_{\text {int }}=E_{\text {zeolite }, T S}-\left(E_{T S, g a s}+E_{\text {zeolite }, \text { blank }}\right)
$$

Therefore, in order to isolate the influence of the zeolite upon the transition state complex, the influence of the zeolite at the reactant state $\left(\Delta \mathrm{E}_{\mathrm{CFE}}\right)$ was subtracted from the total influence of the zeolite at the transition state $\left(\Delta \mathrm{E}_{\text {int }}\right)$ to yield a referenced interaction energy $\left(\Delta \mathrm{E}_{\mathrm{int}, \mathrm{ref}}\right)$.

$$
\Delta E_{\text {int }, \text { ref }}=\Delta E_{\text {int }}-\Delta E_{C F}
$$

By subtracting out the $\Delta \mathrm{E}_{\mathrm{CFE}}$, the referenced interaction energy $\Delta \mathrm{E}_{\text {int,ref }}$ describes both the zeolite's effect on the reactant state as well as the transition state, and gives a better representation of the total zeolite effect on the apparent free energies of activation. 


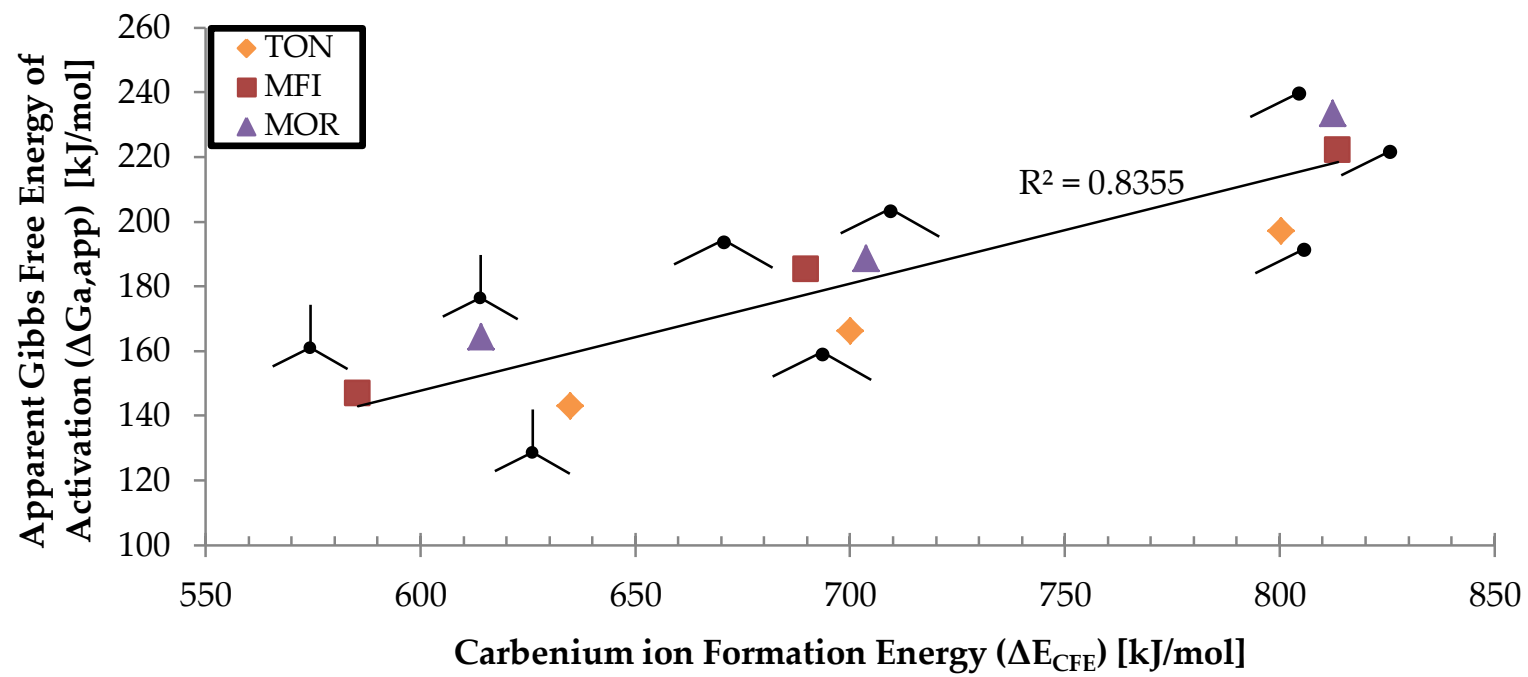

Figure 3.14.2: Comparison of carbenium ion formation energy with apparent Gibbs free energy of activation for TON, MFI, and MOR $(\mathrm{T}=600 \mathrm{~K})$.

The dealkylation of the alkoxy from the zeolite shown in Figure 3.14.2 illustrate similar conclusions to those reached in the gas phase analysis from section 3.7 regarding degree of substitution and alkoxy stability. However, due to differences in the site model, the carbenium ion formation energies calculated with Born-Haber cycle analysis are higher than those calculated for gas phase $\mathrm{C}^{*}-\mathrm{O}$ activation energies, which used the simple single-site cluster. The zeolite framework has additional secondary interactions with the $\mathrm{H}$ on the methyl ligands and the $\mathrm{O}^{*}$ on the neighboring $\mathrm{Al}-\mathrm{O}^{*}$ sites, which are not present in the single-site cluster. More importantly, the hydride capped single-site cluster decreases the overall basicity of the single $\mathrm{Al}-\mathrm{O}^{*}$ site, which weakens the $\mathrm{C}^{*}-\mathrm{O}$ bond and lowers the reaction energy barrier for carbenium ion formation. Jones et al. compared calculated DPE values from density functional theory with experimental estimates of acid strength using the Gaussian code and showed that larger clusters consisting of $>20$ T-atoms significantly weakened the calculated DPE and more closely represent the electrostatic interactions found in the actual zeolite lattice. The authors noted a clear change in acidity based upon number of T-Cells used in the model cluster [29]. The 40 $\mathrm{kJ} / \mathrm{mol}$ difference between the single-site cluster and expanded zeolite lattice observed here is thus attributed to the difference in electrostatic interactions between the two models. 
The calculated carbenium ion formation energies agree with those obtained from gas phase experiments in section 3.7, with ethoxy activation yielding the highest carbenium ion formation energy at roughly $800 \mathrm{~kJ} / \mathrm{mol}$, followed by 2-propoxy, and t-butoxy with the lowest carbenium ion formation energy at around $600 \mathrm{~kJ} / \mathrm{mol}$. The difference in alkoxy substitution far outweighs the calculated differences due to zeolite framework. The carbenium ion formation energy between ethoxy and 2-propoxy in MOR differs by $128 \mathrm{~kJ} / \mathrm{mol}$, whereas carbenium ion formation energy for 2-propoxy between TON and MOR differs by $5 \mathrm{~kJ} / \mathrm{mol}$.

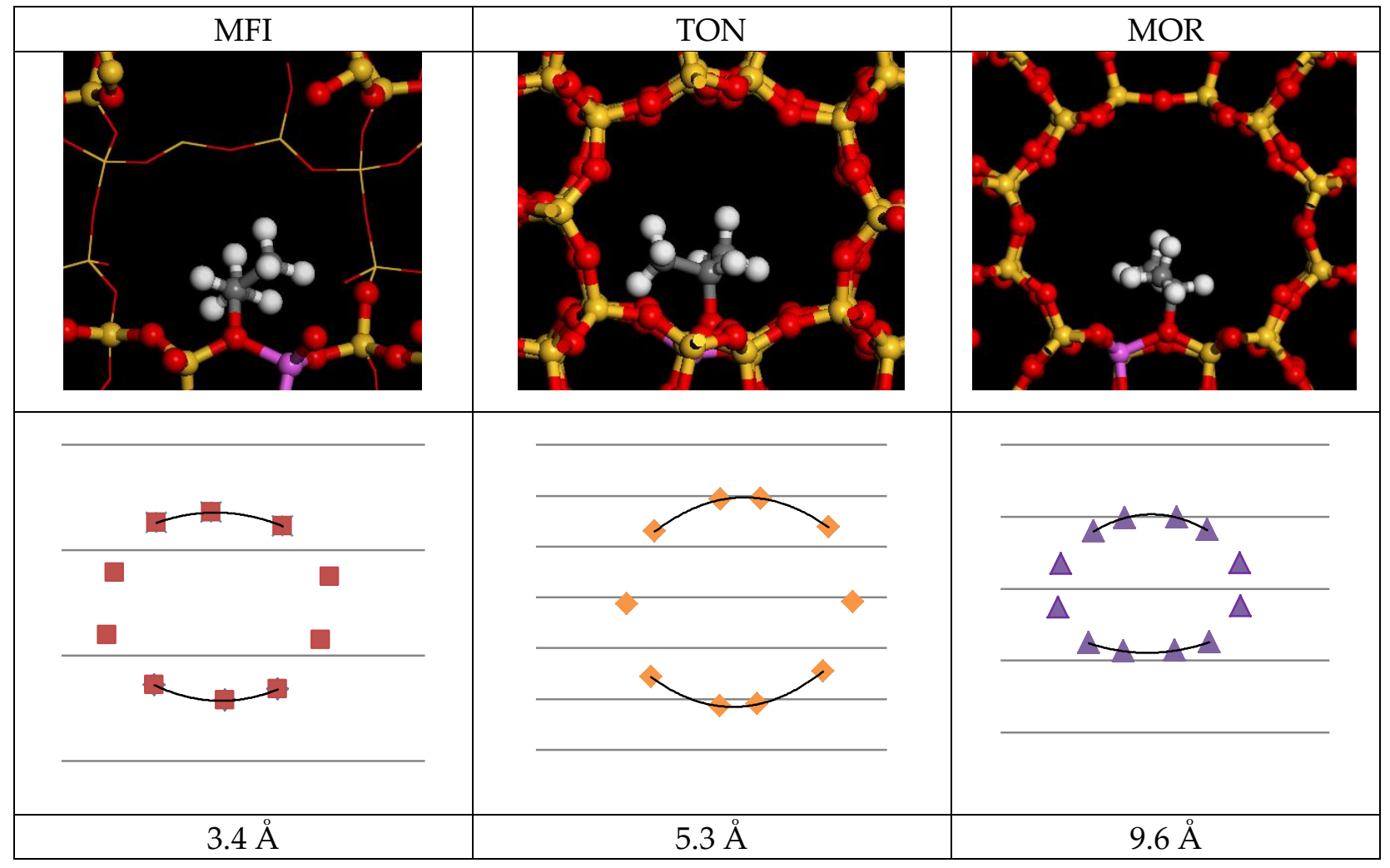

Figure 3.14.3: Fitted radius of curvature estimates for MFI, TON, and MOR.

The differences in carbenium ion formation energies for the different zeolites examined may be the result of differences in curvature, where zeolites with sharp concave pores may result in more repulsive interactions with the bound alkoxide and significantly weaken the $\mathrm{AlO}^{*}-\mathrm{R}$ bond strength. A weaker $\mathrm{AlO}^{*}-\mathrm{R}$ bond will lower the $\triangle \mathrm{E}$ cFE energy and thus assist in carbenium ion formation. The radius of curvature of the dominant zeolite channel for a 
particular zeolite was calculated fitting the atomic-scale coordinates to a parabolic function, the results of which are shown in Figure 3.14.3. The results show that the curvature of TON is much more concave than MOR. Interestingly, MFI yields the smallest radius of curvature of the zeolites tested due to the circular pore shape of MFI, followed by TON and MOR which are both more oval by comparison. However, due to the small size of the adsorbed $\mathrm{C}_{2}-\mathrm{C}_{4}$ alkoxy intermediates, the changes in curvature from one zeolite to another only slightly impact the carbenium ion formation activation.

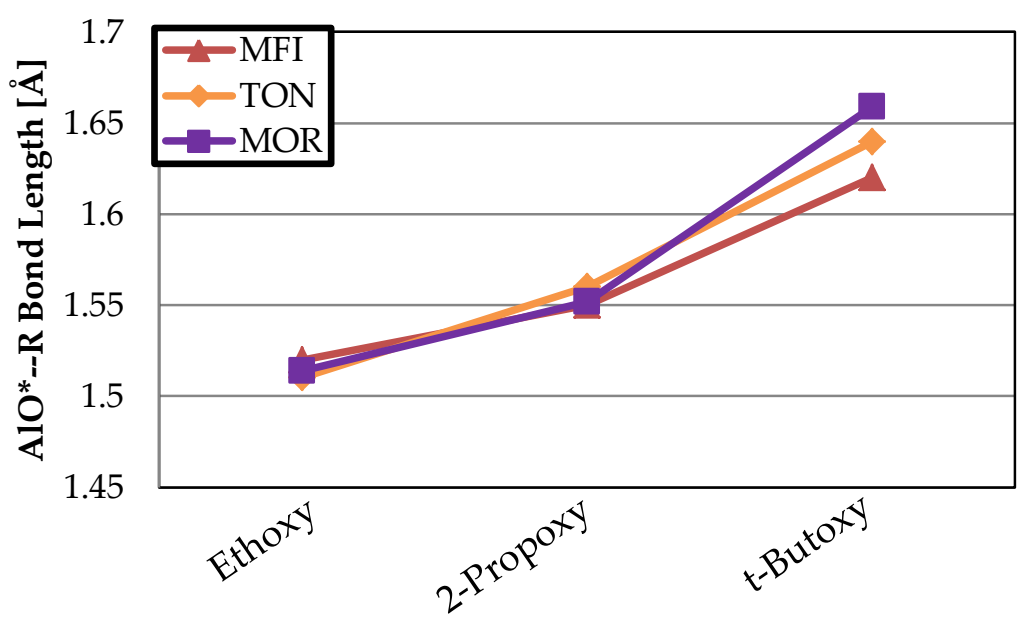

Figure 3.14.4: $\mathrm{AlO}^{*}-\mathrm{R}$ bond lengths for ethoxy, 2-propoxy, and t-butoxy in TON, MFI, and MOR.

A further analysis on the influence of curvature differences between zeolites was done by examining the $\mathrm{AlO}^{*}-\mathrm{R}$ bond lengths of the $\mathrm{C}_{2}-\mathrm{C}_{4}$ alkoxides at the adsorbed alkoxy state. A comparison of $\mathrm{AlO}^{*}-\mathrm{R}$ bond lengths between the carbenium ion carbon and $\mathrm{Al}-\mathrm{O}^{*}$ site shows very little difference in alkoxy $\mathrm{C}^{*}$--O bond length, with the largest variation found for $\mathrm{t}$-butoxy at approximately $0.04 \AA$ (Figure 3.14.4). Comparisons over zeolites with different vdW volume overlap also show little difference in total interaction to the adsorbed alkoxy state due to geometric differences in overall zeolite shape. It therefore follows that systems with higher degrees of substitution for small alkoxides have a significantly stronger effect on the carbenium ion formation energy than the differences that result in zeolite shape between TON, MOR, and MFI. 

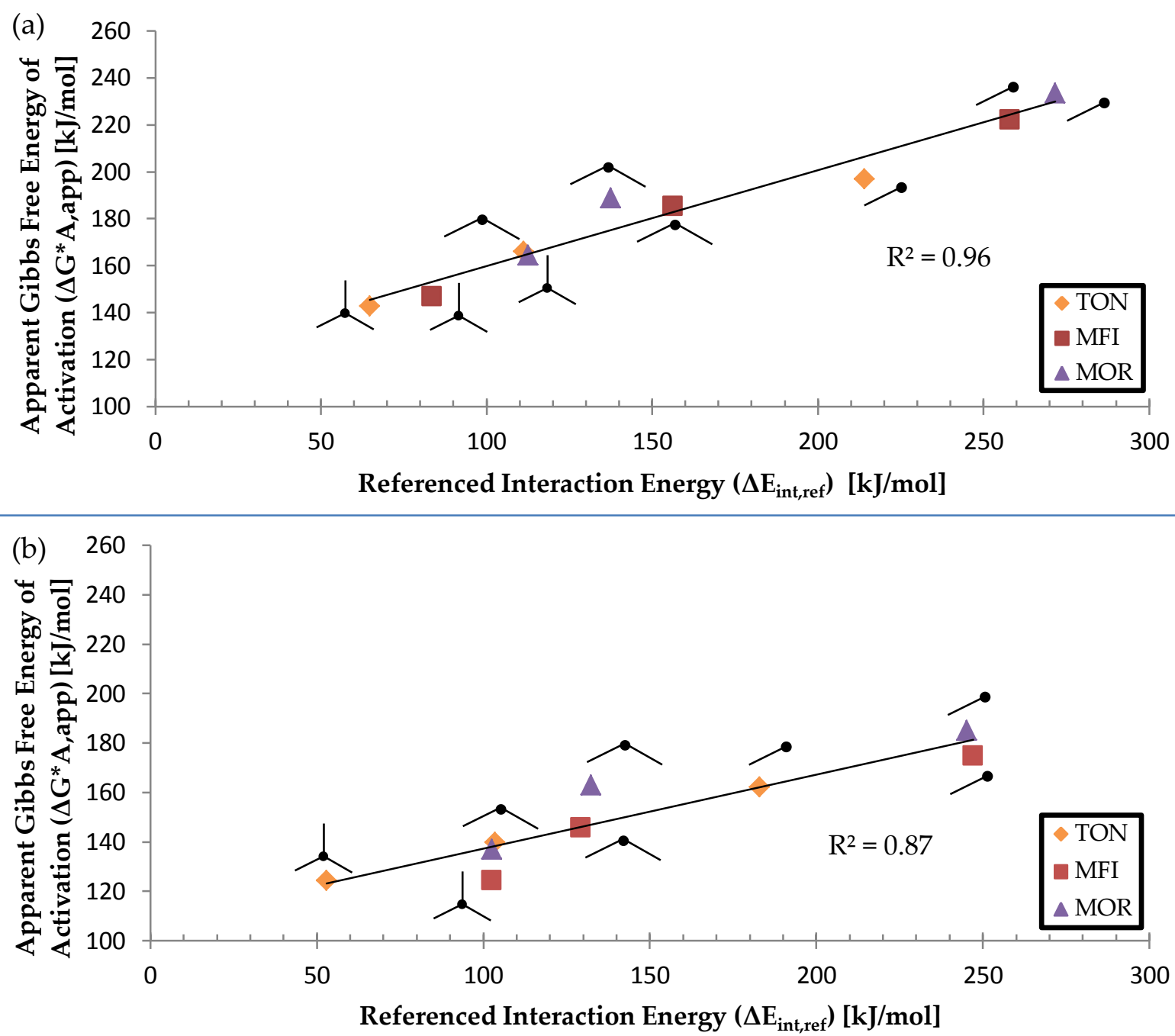

Figure 3.14.5: Relationship between apparent Gibbs free energy of activation and calculated referenced zeolite-transition state interaction energy for (a) propene oligomerization and (b) isobutene oligomerization in TON, MFI, and MOR $(\mathrm{T}=600 \mathrm{~K})$.

All calculated referenced interaction energies are positive, emphasizing the significant stability of the adsorbed alkoxy state prior to reaction, in comparison to the energy gained from the stabilizing secondary interactions at the adsorbed transition state (Figure 3.14.5). The referenced interaction energy shows a very good linear correlation with apparent Gibbs free energies of activation, with the lower interaction energies correlating with lower apparent activation energies, and signifying less stable reactant states and more stable transition states. 
As the alkoxy moves from ethoxy to t-butoxy, the referenced interaction energy decreases by roughly $50-100 \mathrm{~kJ} / \mathrm{mol}$ due to the increased stability provided by the higher order carbenium ion centers as shown with the carbenium ion formation energy. As the transition state complex increases in size, the difference in calculated referenced interaction energy decreases, emphasizing the increase in favorable secondary interactions with larger transition state complexes that is otherwise not observed in just the carbenium ion formation energy. The differences in carbenium ion formation energy in MFI for $C_{4}$ to $C_{3}$ and $C_{3}$ to $C_{2}$ alkoxides are 131 $\mathrm{kJ} / \mathrm{mol}$ and $83 \mathrm{~kJ} / \mathrm{mol}$, respectively, whereas differences in referenced interaction energy differences between $C_{2}$ to $C_{3}$ and $C_{3}$ to $C_{4}$ alkoxides are $117 \mathrm{~kJ} / \mathrm{mol}$ and $27 \mathrm{~kJ} / \mathrm{mol}$, respectively, for isobutene oligomerization in MFI.

Comparisons across zeolites show that TON consistently results in more favorable referenced interaction energies, followed by MFI and MOR, reflecting the differences in secondary interactions of the transition state complexes with the surrounding zeolite framework. In propene oligomerization, MFI exhibits slightly more referenced interaction energy than MOR, with differences ranging from $29 \mathrm{~kJ} / \mathrm{mol}$ to $14 \mathrm{~kJ} / \mathrm{mol}$ between MFI and MOR referenced interaction energies. It is unlikely that this more favorable interaction derives from differences in curvature at the Al--O* site and smaller chamber size in MFI in comparison to MOR, as the differences in carbenium ion formation energy are fairly minimal, and it is therefore due almost entirely to differences in the interactions between the smaller transition state complexes and the surrounding zeolite framework.

This hypothesis concerning more favorable referenced interaction energy with decreasing zeolite size is further supported in the case of isobutene oligomerization. While TON exhibits the most favorable referenced interaction energies, the values calculated for MFI and MOR show little discernable difference, with the largest difference observed for ethoxyisobutene oligomerization at $2 \mathrm{~kJ} / \mathrm{mol}$. The larger transition state complexes are able to interact favorably with the larger MOR framework, and the calculated interaction energy becomes comparable to those calculated for MFI. 


\subsection{Concluding Remarks}

The results reported herein shows that the relative differences in stabilization of the reacting carbenium ion by the surrounding zeolite framework as well as differences in alkene adsorption largely determine the overall activity for alkene oligomerization in various zeolite structures. The activity for alkene oligomerization among the gas phase molecules examined are determined by inherent stability of the carbenium ion intermediates that form during reaction as well as delocalization ability for the reacting alkene to stabilize the resulting positive charge on the resulting dimerized intermediate. For alkene oligomerization within a particular zeolite, the proximity of the transition state complex to the zeolite framework enables an additional stabilization of positive charge throughout the reaction via secondary interactions with both the carbenium ion intermediate and reacting alkene. In particular, interaction with the carbenium ions formed during oligomerization is found to be crucial for oligomerization activity, as the Born-Haber cycle analysis found that more stable carbenium ions yield lower relative free energies of activation for alkene oligomerization. TON is found to best stabilize the transition state during reaction as the narrow 1-D channel maximizes the number of potential secondary interactions with the carbenium ion throughout the oligomerization reaction. This effect of carbenium ion stability on catalyst activity holds large similarity to the importance of enolate stability for aldol condensation, as both aldol condensation and alkene oligomerization demonstrate that the catalyst's potential to further stabilize a charged intermediate largely determines the activity of the catalyst for the reaction.

Outside of secondary interactions, comparisons across zeolites for alkene oligomerization yield only small differences in charge transfer during carbenium ion formation

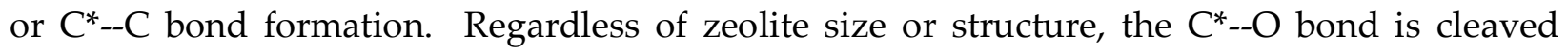
heterolytically in exactly the same way relative to a normalized reaction coordinate, with no perceivable differences in localized charge magnitude on the zeolite, alkene, or carbocation fragments at any point during carbenium ion formation. The only differences in charge magnitude are observed between zeolites following the transition state, but they are minor with differences of less than 0.1 e-. The work reported herein concludes that the shape of the zeolite has no direct coulombic effect on the separation of charge during $\mathrm{C}^{*}$---O bond cleavage that 
otherwise stabilizes or destabilizes the carbenium ion intermediate differently between zeolites. Rather, the largest effect of the zeolite during alkene oligomerization is in the secondary interactions with the alkene during physisorption and with the carbenium ion and transition state complex during alkene oligomerization. The sums of these minor interactions are what set the significant differences in activity of the zeolite for alkene oligomerization.

\section{$\underline{3.16 \text { References }}$}

[1] Bellussi, G.; Pollesel, P., "Industrial applications of zeolite catalysts: Production and uses of light olefins", Stud. Surf. Sci. Catal. 2005, 158, 1201-1212.

[2] Kiricsi, I.; Pálinkó, I.; Kollár, T., “An UV-Vis spectroscopic study on carbenium ions formed on HY-FAU zeolite upon the adsorption of various hydrocarbons", J. Mol. Struct. 2003, 651-653, 331-334.

[3] Janik, M.; Davis, R.; Neurock, M., “A quantum chemical study of tertiary carbenium ions in acid catalyzed hydrocarbon conversions over phosphotungstic acid", Catal. Today 2006, 116, 90-98.

[4] Janik, M.; Davis, R.; Neurock, M., “A density functional theory study of the alkylation of isobutene with butane over phosphotungstic acid", J. Catal, 2006, 244, 65-77.

[5] Derouane, E., "Shape selectivity in catalysis by zeolites: The nest effect", J. Catal. 1986, $100,541-544$.

[6] Derouane, E.; André, J.; Lucas, A., "Surface curvature effects in physisorption and catalysis by microporous solids and molecular sieves", J. Catal. 1988, 110, 58-73.

[7] Derouane, E.; André, J.; Lucas, A., “A simple van der Waals model for molecule-curved surface interactions in molecular-sized microporous solids", Chem. Phys. Let. 1987, 137, 336-340.

[8] Lucas, A.; Derycke, I.; Lambin, P.; Vigneron, J.; Leherte, L.; Elanany, M.; André, J.; Larin, A.; Vercauteren, D., "Confinement in molecular sieves: The pioneering physical concepts", J. Mol. Catal. A: Chem. 2009, 305, 16-23. 
[9] Tanchoux, N.; Pariente, S.; Trens, P.; Fajula, F., “Confinement and curvature effects as a tool for selectivity orientation in heterogeneous catalysis: Isomerization of n-hexene over MCM-41-type catalysts", J. Mol. Catal. A: Chem. 2009, 305, 8-15.

[10] Luo, J.; Bhaskar, B.; Yeh, Y.; Gorte, R., "n-Hexane cracking at high pressures on H-ZSM5, H-BEA, H-MOR, and USY for endothermic reforming", Appl. Catal. A: Gen 2014, 478, 228-233.

[11] Smit, B.; Maesen, T. L. M., "Molecular simulations of Zeolites: Adsorption, diffusions, and shape selectivity", Chem. Rev. 2008, 108, 4125-4184.

[12] Pantu, P.; Boekfa, B.; Limtrakul, J., "The adsorption of saturated and unsaturated hydrocarbons on nanostructured zeolites (H-MOR and H-FAU): An ONIOM study", J. Mol. Catal. A: Chem. 2007, 277, 171-179.

[13] Corma, A.; Martínez, C.; Doskocil, E., “Designing MFI-based catalysts with improved catalyst life for $\mathrm{C}_{3}{ }^{=}$and $\mathrm{C}_{5}=$ oligomerization to high-quality liquid fuels", J. Catal. 2013, $300,183-196$.

[14] Mlinar, A.; Zimmerman, P.; Celik, F.; Head-Gordon, M.; Bell, A., "Effects of Brønstedacid site proximity on the oligomerization of propene in H-MFI", J. Catal. 2012, 288, 6573.

[15] Kresse, G.; Furthmüller, J., "Efficient iterative schemes for ab initio total-energy calculations using a plane-wave basis set", Phys. Rev. B 1996, 54, 11169-11186.

[16] Grimme, S., "Semiempirical GGA-type density functional constructed with a long-range dispersion correction", J. Comp. Chem. 2006, 27, 1787-1799.

[17] Audouze, C.; Jollet, F; Torrent, M.; Gonze, X., “Comparison between projector augmented-wave and ultrasound pseudopotential formalisms at the density-functional perturbation theory level", Phys. Rev. B 2008, 78, 035105.

[18] Tang, W.; Sanville, E.; Henkelman, G., "A grid-based Bader analysis algorithm without lattice bias", J. Phys-Condens. Mat. 2009, 21, 084204.

[19] Henkelman, G.; Uberuaga, B. P.; Jónsson, H., “A climbing image nudged elastic band method for finding saddle points and minimum energy paths", J. Chem. Phys. 2000, 113, 9901-9904. 
[20] Henkelman, G.; Jónsson, H., “Improved tangent estimate in the nudged elastic band method for finding minimum energy paths and saddles points", J. Chem. Phys. 2000, 113, 9978-9985.

[21] Siepmann, J. I.; Frenkel, D., “Configurational bias Monte Carlo: A new sampling scheme for flexible chains", Mol. Phys. 1992, 75, 59-70.

[22] Martin, M.; Siepmann, J., “Novel configurational-bias Monte Carlo method for branched molecules. Transferable potentials for phase equilibria. 2. United-atom description of branched alkanes", J. Phys. Chem. B, 1999, 103, 4508-4517.

[23] Wick, C.; Martin, M.; Siepmann, J., “Transferable potentials for phase equilibria. 4. United-atom description of linear and branched alkenes and alkylbenzenes", J. Phys. Chem. B 2000, 104, 8008-8016.

[24] Chen, B.; Potoff, J. J.; Siepmann, J., "Monte Carlo calculations for alcohols and their mixtures with alkanes. Transferable potentials for phase equilibria. 5. United-atom description of primary, secondary, and tertiary alcohols", J. Phys. Chem. B 2001, 105, 3093-3104.

[25] Bai, P.; Tsapatsis, M.; Siepmann, J., “TraPPE-zeo: Transferable potentials for phase equilibria force field for all-silica zeolites", J. Phys. Chem. C 2013, 117, 24375-24387.

[26] Dassault Systèmes BIOVIA, Materials Studio, 6.1, San Diego: Dassault Systèmes, 2014.

[27] Ch. Baerlocher and L.B. McCusker, Database of Zeolite Structures: http://www.izastructure.org/databases.

[28] Ying, L.; Zhu, J.; Cheng, Y.; Wang, L., Li, X., "Kinetic modeling of $\mathrm{C}_{2}-\mathrm{C}_{7}$ olefins interconversion over ZSM-5 catalyst", J. Ind. Eng. Chem, 2016, 33, 80-90.

[29] Jones, A; Carr, R.; Zones, S.; Iglesia, E., “Acid strength and solvation in catalysis by MFI zeolites and effects of the identity, concentration and location of framework heteroatoms", J. Catal. 2014, 312, 58-68. 
CHAPTER 4: Dimethoxymethane Formation in Chabazite

4.1 Deactivation during Methanol-to-Olefins Reaction and Dimethoxymethane Formation in $\underline{\mathrm{CHA}}$

The Methanol-to-Olefins (MTO) reaction provides a direct route for the formation of olefins from methanol. Both olefin oligomerization and MTO proceed via the formation of a carbenium ion intermediate that reacts with olefins to form longer chain hydrocarbons. The predominant difference is MTO proceeds via the formation of a primary $\mathrm{CH}_{3}(+)$ carbenium ion and is partially stabilized by water. Olefin oligomerization, on the other hand, proceeds via the formation of secondary or tertiary carbenium ions that react directly with the olefin. Both reactions show important secondary interactions with the zeolite framework.

Wei et al. carried out theoretical calculations to analyze paths involved in the formation of the initial $\mathrm{C}^{*}-\mathrm{C}$ bond in MTO over H-ZSM-5 [1] and attempted to understand whether the bare Brønsted acid site or the adsorbed carbenium ion was critical for the MTO mechanism. Calculated Gibbs free energies of proposed reaction pathways involving the Brønsted acid site were compared to those involving a chemisorbed carbenium intermediate, and found that the carbenium ion route was kinetically and thermodynamically favored for the proposed concerted H-transfer and $\mathrm{C}^{*}$-C bond formation step between a chemisorbed carbocation with a physisorbed $\mathrm{CH}_{4}, \mathrm{CH}_{3} \mathrm{OH}$, or $\mathrm{CH}_{3} \mathrm{OCH}_{3}$. Their results show that, similar to alkene oligomerization, the formation and reaction of the carbenium ion is crucial to the formation of $\mathrm{C}^{*}-\mathrm{C}$ bonds for the MTO reaction in zeolites.

Zhang et al. carried out DFT cluster calculations to examine the influence of zeolite solvation and acid strength on catalyst activity for methanol-to-olefins conversion in H-ZSM-5 [2]. They examined the influence of different size clusters and suggested that the zeolite framework helped to stabilize the transition states for several elementary reactions involved in MTO. Isosurface plots of reduced density gradient for various MTO transition states were used to visualize the interactions between the transition state and surrounding zeolite. These plots showed significant van der Waal (vdW) interactions for methylation, isomerization, and C5* cracking transition states, further supporting the influence of zeolite framework on transition state stability. Ilias and Bahn analyzed six major reactions in the aromatic- and olefin-based catalytic cycles for the zeolite catalyzed methanol-to-hydrocarbons system, including 
methylation, cracking, and cyclization [3]. Through detailed comparisons of the previous computational and experimental work, they highlighted the importance of carbenium ion stability in the reactions with olefins as well as the influence of zeolite framework on the observed product selectivity.

While MTO is an important route to light alcohol upgrading, the zeolites used readily deactivate as the result of coke formation. The catalytic features that facilitate coke formation as well as the nature of the coke that is formed have been studied to some degree. Catizzone et al. analyzed the catalytic performance of various zeolites for dimethyl ether formation via methanol dehydration, including MOR, beta, SAPO-34, and ferrierite zeolites [4]. They showed significant deactivation via heavy carbon deposits in zeolites with large 3-dimensional channel system such as beta, and those zeolites consisting of 1-D channel structures with large openings or side pockets such as MOR. SAPO-34, the phosphate equivalent of the chabazite (CHA) zeolite, exhibited $>90 \%$ dimethyl ether selectivity, but suffered heavily from deactivation within the first hour on stream. Chen, Miljord, and Holmen [5] noted that the large chamber and narrow pores of SAPO zeolites facilitated the formation of coke with large crystal size and high site densities increasing rate of deactivation when comparing SAPO and CHA zeolites. They identified two possible routes for coke formation: aromatics formed from olefins resulting from the MTO reaction, and coke formed directly from the methanol/dimethyl ether mixture.

Additional studies have also noted the formation of coke from early oxygenate coupling reactions that rapidly deactivate the zeolite catalyst. Müller et al. analyzed the deactivation by coke formation on H-ZSM-5 for MTO reactions [6]. By analyzing the different coke deposits, they showed that early stages of coking were the result of coupling and dehydration of reactant oxygenates that form larger molecules and further react with olefins to form localized aromatics. Site-blocking, rather than pore-blocking, was concluded to be the cause for loss of activity for MTO reactions. Pérez-Uriate et al. examined the deactivation of H-ZSM-5 during the conversion of dimethyl ether to olefins via kinetic analysis of the coke formed from the reactions of methanol and dimethyl ether to larger molecules [7]. In particular, the formation of methanol and methoxy species from dimethyl ether was identified as a key reason for deactivation in H-ZSM-5. Reactions with the methoxy species, as well as condensation of 
reactant oxygenates, were attributed to the rate of coke formation during the reaction of dimethyl ether to olefins, and coking rates were found to increase with increasing temperature and oxygenate concentration.

Dimethoxymethane (DMM) is a side reaction product that forms during the reaction of methanol to olefins in zeolites and may ultimately offer insights into the oxygenate coupling reactions that result in deactivation. DMM formation is thought to proceed through a hydride transfer from methanol to adsorbed methoxy to form a protonated formaldehyde, C-O coupling of the protonated formaldehyde with a second methanol and subsequent dehydration to form the methoxymethyl intermediate. The methoxymethyl then undergoes oligomerization with additional methanol to form the DMM product. The reverse reaction of DMM to methanol and methoxymethyl intermediate has already been observed in literature. Celik et al. investigated the mechanism and kinetics of carbonylation of DMM to form methyl methoxyacetate in FAU and MFI zeolites [8]. Of the results collected, in situ IR spectroscopy revealed the formation of methanol and chemisorbed methoxymethyl species from physisorbed DMM at Brønsted acid sites. In addition, IR analysis revealed methoxymethyl to be the primary adsorbed species, with bands for other chemisorbed species making small peaks or shoulders to the methoxy methyl band.

Celik, Kim, and Bell examined the effect of zeolite framework and Si/Al ratio on DMM carbonylation using catalytic ratio and selectivity analysis [9]. In particular, the high selectivity in FAU was found to derive from the large super-cages that reduced DMM disproportionation in comparison to the smaller MFI cage that facilitated the side reactions due to forced close proximity of the reactants. They also proposed that the low $\mathrm{Si} / \mathrm{Al}$ ratios that resulted in decreased carbonylation rates was due to molecular crowding via intermolecular electrostatic repulsion from multiple catalytic sites within a single zeolite cage. The results from this work on the effect of zeolite shape on the DMM reactions and MTO deactivation indicate that the CHA type zeolite can readily carry out DMM formation reaction, and may provide insights into the mechanism that control the initial deactivation routes in zeolites.

While significant work regarding deactivation pathways for MTO in zeolites have revealed the crucial effects of zeolite size and oxygenate concentration, detailed mechanistic 
analysis of DMM formation as well as the effects facilitating methanol coupling for deactivation have not been studied. The work herein analyzes the proposed mechanism for dimethoxymethane (DMM) formation in CHA zeolite as a model reaction in order to better understand those governing features for one of the possible starting oxygenate coupling reactions that may participate in the deactivation of zeolites during the methanol-to-olefin reaction.

\subsection{Dimethoxymethane Formation Mechanism}

The reaction path and proposed mechanism for dimethoxymethane (DMM) formation occurs via the reactions of methanol and adsorbed methoxy intermediate within the zeolite. The reaction proceeds via a hydride transfer from the gas phase methanol to the adsorbed methoxy, to form methane which leaves in the gas phase and the hydroxymethyl $\left({ }^{+} \mathrm{H}_{2} \mathrm{C}-\mathrm{OH}\right)$ carbenium intermediate (Scheme 4.2.1) that subsequently adsorbs onto the Al-O* oxygen site to form the bound hydroxymethyl alkoxide $\left(\mathrm{H}_{2} \mathrm{C}^{*}(\mathrm{OH})-\mathrm{OAl}\right)$. A second methanol subsequently physisorbs and reacts with the adsorbed ${ }^{+} \mathrm{H}_{2} \mathrm{C}-\mathrm{OH}$ to form $\mathrm{C}-\mathrm{O}$ bond between the $\mathrm{O}$ on the alcohol and the $\mathrm{C}$ of the ${ }^{+} \mathrm{H}_{2} \mathrm{C}-\mathrm{OH}$. This is followed by rapid deprotonation to form the $\mathrm{CH}_{3} \mathrm{OCH}_{2} \mathrm{OH}$ hemiacetal intermediate and zeolite Brønsted acid site. Dehydration of the hemiacetal yields water and the $\mathrm{CH}_{3} \mathrm{OCH}_{2}{ }^{+}$intermediate, and the final $\mathrm{C}-\mathrm{O}$ bond formation and deprotonation with a third methanol molecule which results in the formation of the DMM product and the zeolite Brønsted acid site. Two of the zeolite oxygen sites are proposed to participate in the reaction to reabsorb the intermediate molecules immediately following each elementary reaction in order to stabilize intermediates with charged carbon centers.

$$
\begin{aligned}
& Z O-H+\boldsymbol{C H}_{\mathbf{3}} \boldsymbol{O} \boldsymbol{H}_{(\boldsymbol{a d s})(\mathrm{gas})} \stackrel{k_{\text {meth }}}{\rightleftharpoons} \mathrm{ZO}-\mathrm{CH}_{3}+\mathrm{H}_{\mathbf{2}} \boldsymbol{O}_{(\boldsymbol{a d s})(\mathrm{gas})} \\
& \mathrm{ZO}-\mathrm{CH}_{3}+\mathbf{C H}_{3} \boldsymbol{O H}_{(\text {ads })(\mathrm{gas})} \stackrel{k_{\mathrm{HT}}}{\rightleftharpoons} \mathrm{ZO}-\mathrm{CH}_{2} \mathrm{OH}+\mathbf{C H}_{\mathbf{4}(\text { ads })(\mathrm{gas})} \\
& \mathrm{ZO}-\mathrm{CH}_{2} \mathrm{OH}+\mathbf{C H}_{3} \mathbf{O H}_{(\boldsymbol{a d s})(\mathrm{gas})} \stackrel{k_{\text {hemi }}}{\rightleftharpoons} \mathrm{CH}_{3} \mathrm{OCH}_{2} \mathrm{OH}_{(\text {ads })(\text { gas })}+\mathrm{ZO}-\mathrm{H} \\
& \mathrm{CH}_{3} \mathrm{OCH}_{2} \mathrm{OH}_{(\text {ads })(\mathrm{gas})}+\mathrm{ZO}-\mathrm{H} \stackrel{k_{\text {dehy }}}{\rightleftharpoons} \mathrm{ZO}-\mathrm{CH}_{2} \mathrm{OCH}_{3}+\boldsymbol{H}_{2} \boldsymbol{O}_{(\text {ads })(g a s)} \\
& \mathrm{ZO}-\mathrm{CH}_{2} \mathrm{OCH}_{3}+\mathrm{CH}_{3} \mathrm{OH}_{(\text {ads })(\text { gas })} \stackrel{k_{D M M}}{\rightleftharpoons} \mathrm{CH}_{3} \mathrm{OCH}_{2} \mathrm{OCH}_{3(\text { ads })(g a s)}+\mathrm{ZO}-\mathrm{H}
\end{aligned}
$$

Scheme 4.2.1: Proposed mechanism for DMM formation, where ZO signifies zeolite oxygen site 


\section{$\underline{4.3 \text { Computational Methods }}$}

All of the calculations reported herein were carried out using periodic dispersioncorrected density functional theory (DFT-D2) calculations as implemented in the Vienna ab initio Simulation Package (VASP) [10]. The effects of dispersion were accounted for through the use of semi-empirical Grimme-D2 corrections [11]. The Perdew-Burke-Ernzerhof (PBE) exchange-correlation functional was used to calculate nonlocal gradient corrections to exchange and correlation energies. Projector-augmented wave (PAW) pseudopotentials were used to describe core electrons [12]. Valence electrons were described with a plane-wave basis set with an energy cutoff of $396 \mathrm{ev}$. The wave functions and charge density were converged to within $10^{-6} \mathrm{eV}$. Due to the large unit-cell sizes and the localized nature of the valence electrons of the zeolites, the sampling of the Brillouin zone was done at the $\gamma$ point only. All atoms were allowed to relax during geometric optimization with a force criteria and energy criteria for optimization set at $0.05 \mathrm{eV} / \AA$ and $10^{-6} \mathrm{eV}$, respectively. Atomic charges were analyzed using the Bader charge analysis [13] which describes the charge distribution by partitioning of the electron density.

The saddle points were initially located using the nudged elastic band method (NEB) to map out the reaction coordinate $[14,15]$. Thirty two evenly spaced points were chosen along the reaction coordinate and the structures at each of these points were minimized until the forces perpendicular to the reaction path converged to within $0.25 \mathrm{eV} / \AA$. The minimum energy reaction path and the approximate transition states were subsequently used as input to isolating more refined transition states using the dimer method [15]. The dimer calculations were converged until the forces perpendicular to the reaction mode were less than $0.05 \mathrm{eV} / \AA$.

\subsection{Chabazite Model}

Chabazite (CHA) zeolite is characterized by its moderate chamber formed at intersecting perpendicular channels. Fractional coordinates and lattice parameters of the CHA model (Table 4.4.1) used herein were taken from Accelrys Materials Studio structure library [16] and from the database of zeolite structures [17] (Table 4.4.1). The CHA structure contains 1 unique placement for the Al substitution, with 4 adjacent oxygens uniquely different from one another. The 
zeolite model used herein is a supercell of the rhombohedral lattice type unit expanded $2 \times 2 \times 2$, with final lattice parameters of $\mathrm{a}=\mathrm{b}=\mathrm{c}=18.842$ and $\alpha=\beta=\gamma=94.2$ degrees (Figure 4.4.2).

Table 4.4.1: Lattice parameters and atomic fractional coordinates of optimized CHA zeolite model.

\begin{tabular}{|l|l|}
\hline Space Group & R-3M (\#166) \\
\hline Unit Cell Parameters & $\mathrm{a}=\mathrm{b}=13.80258$ \\
& $\mathrm{c}=15.075288$ \\
\hline & $\alpha=\beta=90^{\circ}$ \\
& $\gamma=120^{\circ}$ \\
\hline
\end{tabular}

\begin{tabular}{|l|l|l|l|}
\hline & $x / a$ & $y / b$ & $z / c$ \\
\hline $\mathrm{Si}$ & 0.666700 & 0.562801 & 0.437700 \\
\hline $\mathrm{O}(1)$ & 0.785633 & 0.571267 & 0.465867 \\
\hline $\mathrm{O}(2)$ & 0.565700 & 0.434300 & 0.459100 \\
\hline $\mathrm{O}(3)$ & 0.654800 & 0.654000 & 0.500100 \\
\hline $\mathrm{O}(4)$ & 0.666667 & 0.597133 & 0.333333 \\
\hline
\end{tabular}
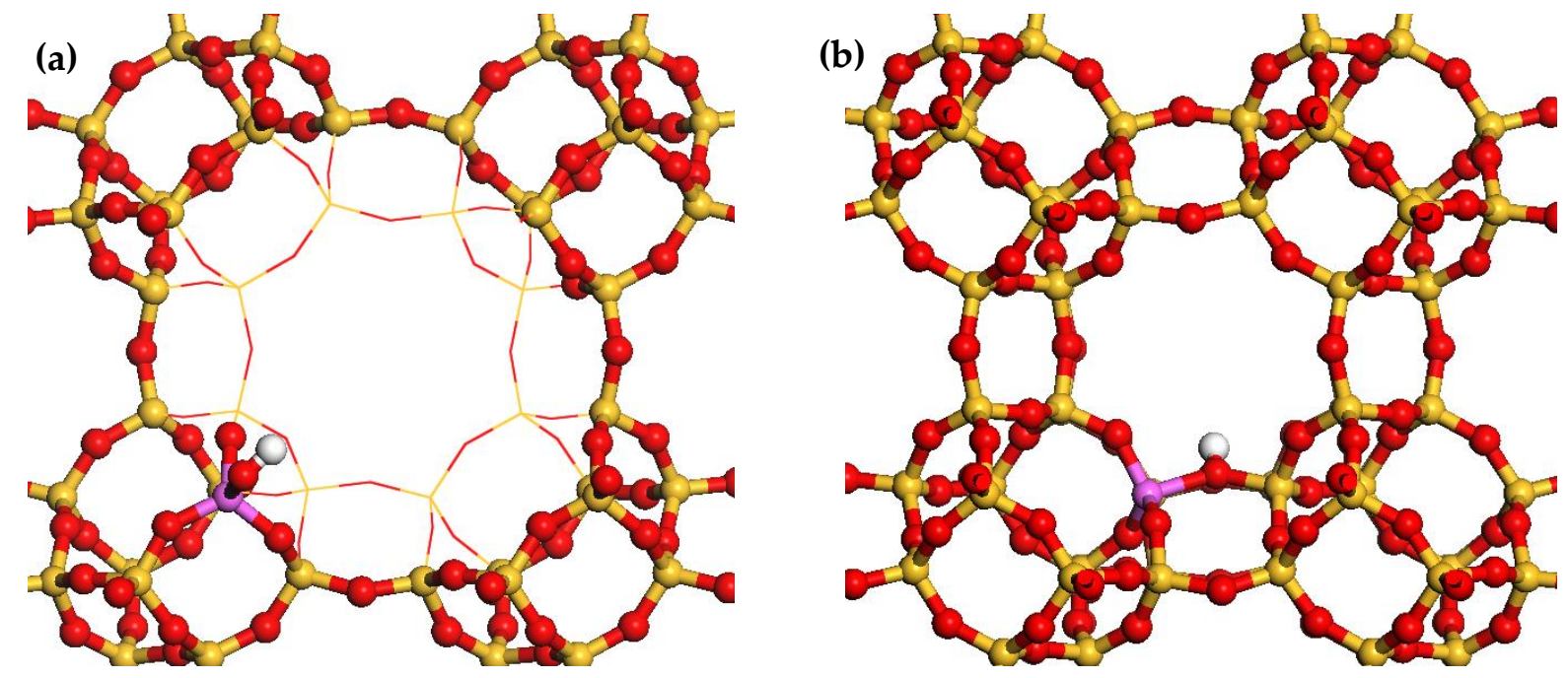

Figure 4.4.2: Chamber view (a) and pore view (b) of CHA model.

\section{$\underline{4.5 \text { Gas Phase Calculations }}$}

Gas phase calculations of the complete catalytic cycle for dimethoxymethane (DMM) formation were performed using Gaussian in order to decompose each of the elementary reaction steps into relevant gas phase thermochemical paths to capture the influence of the 
reactant on reactivity and the relevant interactions of the gas phase intermediates and catalyst that capture the influence of the catalyst (Figure 4.5.1).

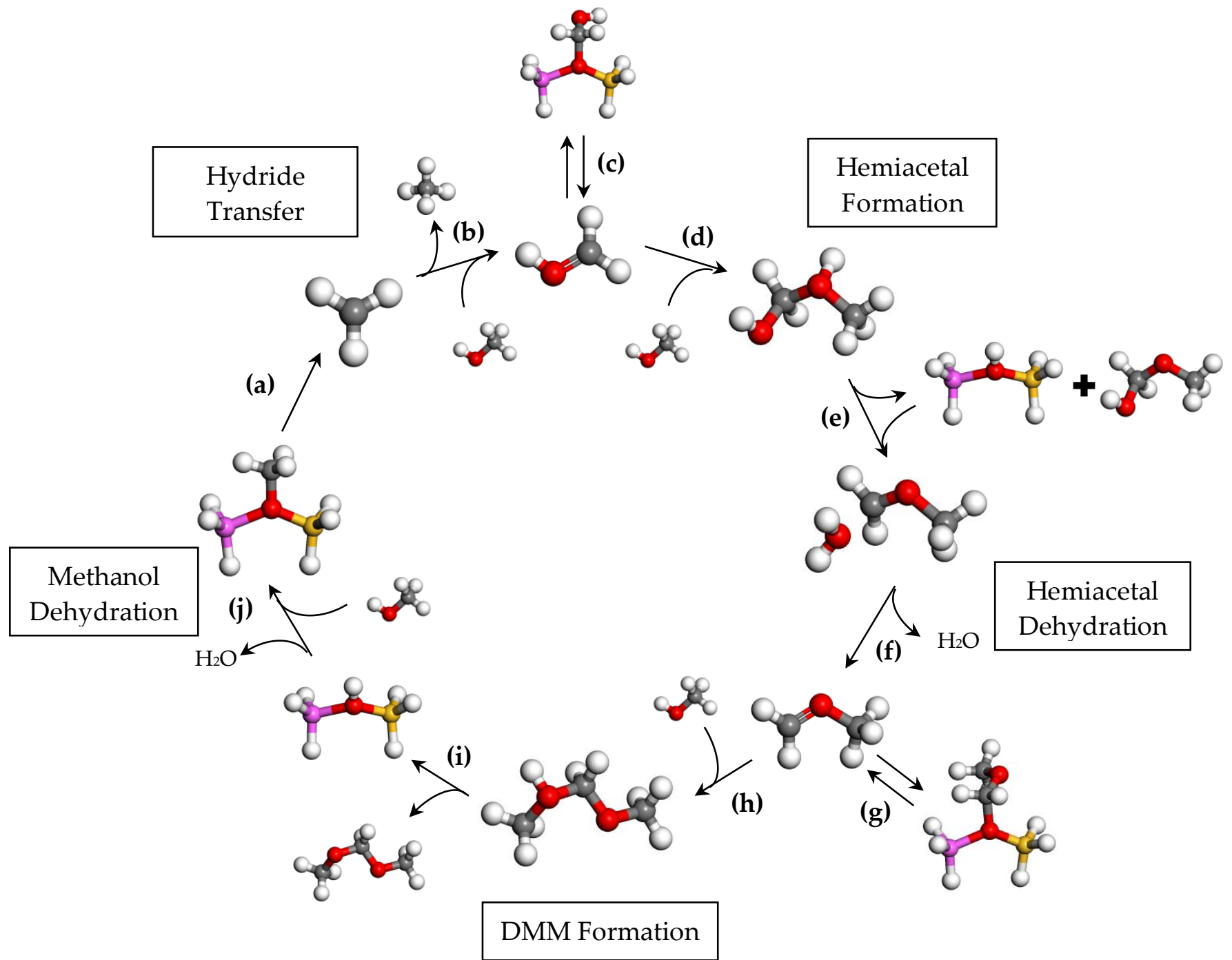

Figure 4.5.1: Catalytic cycle for DMM formation showing (a) methyl ion activation, (b) hydride transfer with methanol, (c) adsorption/desorption of hydroxymethyl, (d) hemiacetal formation with $2^{\text {nd }}$ methanol, (e) deprotonation and deprotonation of hemiacetal, (f) dehydration, (g) adsorption/desorption of methoxymethyl cation, (h) C---O bond formation with $3^{\text {rd }}$ methanol, (i) deprotonation and DMM formation, and (j) site restoration via methanol dehydration. 
DMM formation begins with hydride transfer from a physisorbed methanol to an adsorbed methoxy bound to the $\mathrm{AlO}^{*}$ site within a zeolite, thus yielding the methane product and an adsorbed hydroxymethyl $\left(\mathrm{CH}_{2} \mathrm{OH}(+)\right)$ at a neighboring $\mathrm{AlO}^{*}$ site. This first elementary step involves the formation of a methyl cation via methoxy $\mathrm{AlO}^{*}-\mathrm{CH}_{3}{ }^{*}$ activation, hydride transfer from methanol to the methyl cation, and subsequent hydroxymethyl $\mathrm{AlO}^{*}-\mathrm{C}^{*}$ bond formation. This ultimately occurs in a single concerted step. Methyl cation formation was calculated using the same zeolite cluster used in alkene oligomerization gas phase calculations for consistency and remove any possible solvation effects from the zeolite. As a result, the calculations reported on the cluster will underestimate the $\mathrm{AlO}^{*}-\mathrm{C}^{*}$ energy cost due to the fact that the cluster is capped with hydrides. Even with an underestimated value, methyl carbenium ion formation was found to have the highest energy cost of all the gas phase calculations in DMM formation, with a gas phase energy of $904 \mathrm{~kJ} / \mathrm{mol}$, where the high cost is due to the unstable nature of the gas phase $\mathrm{CH}_{3}{ }^{+}$cation intermediate.

Table 4.5.2: Gas phase energies for hydride transfer elementary step (Figure 4.5.1 (a-c)).

\begin{tabular}{|l|l|}
\hline \multicolumn{2}{|c|}{ Hydride Transfer } \\
\hline Methyl cation formation $\left(\Delta \mathrm{E}_{\mathrm{a}}\right)$ & $904 \mathrm{~kJ} / \mathrm{mol}$ \\
\hline Hydride transfer $\left(\Delta \mathrm{E}_{\mathrm{b}}\right)$ & $-283 \mathrm{~kJ} / \mathrm{mol}$ \\
\hline Hydroxymethyl cation adsorption $\left(\Delta \mathrm{E}_{\mathrm{c}}\right)$ & $-660 \mathrm{~kJ} / \mathrm{mol}$ \\
\hline
\end{tabular}

The methyl cation subsequently reacts with the methanol via a hydride from the $\mathrm{CH}_{3}{ }^{+}$ group of methanol to the methyl cation resulting in a reaction energy of $-283 \mathrm{~kJ} / \mathrm{mol}$ (Table 4.5.2). The oxygen lone pair on the hydroxymethyl allows for increased delocalization of positive charge, and for partial resonance to the reduced aldehyde form which gives additional stability to the $\mathrm{CH}_{2} \mathrm{OH}(+)$ formed, that is otherwise not present in the $\mathrm{CH}_{3}(+)$ intermediate. The stability of $\mathrm{CH}_{2} \mathrm{OH}(+)$ versus that of $\mathrm{CH}_{3}(+)$ makes the hydride transfer step from methanol to methyl cation exothermic at $-283 \mathrm{~kJ} / \mathrm{mol}$.

This effect of the alcohol group on cation stability is further illustrated by comparing the relative energy cost of $\mathrm{AlO}^{*}-\mathrm{R}$ activation between the methoxy and hydromethoxy. The activation of the bound $\mathrm{AlO}^{*}-\mathrm{CH}_{2} \mathrm{OH}^{*}$ with a gas phase reaction energy of $660 \mathrm{~kJ} / \mathrm{mol}$ is more 
favorable than the activation of the bound $\mathrm{AlO}^{*}-\mathrm{CH}_{3}{ }^{*}$ at $744 \mathrm{~kJ} / \mathrm{mol}$. This comparison further emphasizes that the additional stability provided by the lone pair on the hydroxyl group in comparison to the carbocation equivalents.

Table 4.5.3: Gas phase energies for hemiacetal formation elementary step (Figure 4.5.1 (d-e)).

\begin{tabular}{|l|l|}
\hline \multicolumn{2}{|c|}{ Hemiacetal Formation } \\
\hline $\mathrm{C}^{*}$----O bond formation between $\mathrm{CH}_{2} \mathrm{OH}(+)$ and methanol $(\Delta \mathrm{Ed})$ & $-204 \mathrm{~kJ} / \mathrm{mol}$ \\
\hline Deprotonation to form hemiacetal $(\Delta \mathrm{Ee})$ & $-462 \mathrm{~kJ} / \mathrm{mol}$ \\
\hline
\end{tabular}

The second elementary step after hydride transfer in DMM formation involves hemiacetal formation via the reaction between the bound hydroxymethyl intermediate and a $2^{\text {nd }}$ physisorbed methanol molecule to form the $\mathrm{C}^{*}-\mathrm{O}$ bond. The gas phase reaction of the hydroxymethyl cation and methanol is exothermic with a reaction energy of $-204 \mathrm{~kJ} / \mathrm{mol}$ (Table 4.5.3). The $\mathrm{HOCH}_{2}-\mathrm{O}(\mathrm{H}) \mathrm{CH}_{3}(+)$ intermediate subsequently deprotonates to form the hemiacetal intermediate $\mathrm{HOCH}_{2}-\mathrm{O}(\mathrm{H}) \mathrm{CH}_{3}(+)$ and Brønsted acid site at $-462 \mathrm{~kJ} / \mathrm{mol}$. A comparison of the gas phase energies shows that the $\mathrm{AlO}^{*}---\mathrm{CH}_{2} \mathrm{OH}^{*}$ activation at $660 \mathrm{~kJ} / \mathrm{mol}$ has an expectedly higher cost than the energy gained from $\mathrm{C}^{*}---\mathrm{O}$ bond formation between $\mathrm{CH}_{2} \mathrm{OH}(+)$ and methanol at $-204 \mathrm{~kJ} / \mathrm{mol}$. This is expected as $\mathrm{AlO}^{*}--\mathrm{CH}_{2} \mathrm{OH}^{*}$ activation involves taking a stable alkoxy species and desorbing to a form the highly unstable cation, similar to the carbenium ion formation step in alkene oligomerization discussed in chapter 3.

Table 4.5.4: Gas phase energies for hemiacetal dehydration elementary step (Figure 4.5.1 (e-g)).

\begin{tabular}{|l|l|}
\hline \multicolumn{2}{|c|}{ Dehydration of Hemiacetal } \\
\hline Protonation of -OH group on hemiacetal $\left(\Delta \mathrm{E}_{\mathrm{e}}\right)$ & $461 \mathrm{~kJ} / \mathrm{mol}$ \\
\hline Dehydration via $\mathrm{CH}_{3} \mathrm{OCH}_{2}---\mathrm{OH}_{2}$ activation $\left(\Delta \mathrm{E}_{\mathrm{f}}\right)$ & $137 \mathrm{~kJ} / \mathrm{mol}$ \\
\hline Methoxymethyl cation adsorption $\left(\Delta \mathrm{E}_{\mathrm{g}}\right)$ & $-612 \mathrm{~kJ} / \mathrm{mol}$ \\
\hline
\end{tabular}

The dehydration of the hemiacetal can also be broken down into a series of gas phase processes involving the phase energies to include the energy for deprotonation of the 
hemiacetal, gas phase dehydration, and then binding of the resulting methoxymethyl cation. The calculated energies for these steps reported in Table 4.5.4 show that the gas phase $\mathrm{CH}_{3} \mathrm{OCH}_{2}---\mathrm{OH}_{2}$ bond activation that occurs in the dehydration reaction to form water is 137 $\mathrm{kJ} / \mathrm{mol}$, which is significantly smaller than both the protonation energy of the hemiacetal by the Brønsted acid site at $461 \mathrm{~kJ} / \mathrm{mol}$ as well as the binding energy of the resulting methoxymethyl cation $\left(\mathrm{AlO}^{*}-\mathrm{CH}_{2} \mathrm{OCH}_{3}{ }^{*}\right)$ at $-612 \mathrm{~kJ} / \mathrm{mol}$.

Table 4.5.5: Gas phase energies for DMM formation elementary step (Figure 4.1.1 (h-i)).

\begin{tabular}{|l|l|}
\hline \multicolumn{2}{|c|}{ DMM Formation } \\
\hline Methoxymethyl C*---O bond formation to methanol $(\Delta \mathrm{Eh})$ & $-155 \mathrm{~kJ} / \mathrm{mol}$ \\
\hline Deprotonation to form DMM product $(\Delta \mathrm{Ei})$ & $-432 \mathrm{~kJ} / \mathrm{mol}$ \\
\hline
\end{tabular}

The final elementary step in the formation of DMM involves the addition of a third methanol molecule to the bound methoxymethyl $\left(\mathrm{AlO}^{*}-\mathrm{CH}_{2} \mathrm{OCH}_{3}\right)$ and the formation of the

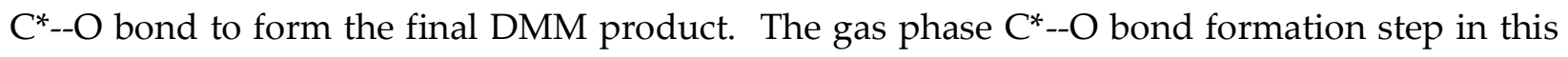
reaction is also rather small at $-155 \mathrm{~kJ} / \mathrm{mol}$, in comparison with the Brønsted acid site formation at $-432 \mathrm{~kJ} / \mathrm{mol}$ (Table 4.5 .5 ) or methoxymethyl cation formation at $612 \mathrm{~kJ} / \mathrm{mol}$. In every elementary step, the decomposition of gas phase energies are consistently dictated by the stability of charged intermediate species via DMM formation, just as it was stressed in alkene oligomerization and aldol condensation. It should therefore follow that any effect by the CHA catalyst that further stabilizes the cation intermediate during reaction or the charged intermediates after reaction will directly influence the overall catalyst activity for DMM formation.

In the subsequent sections, we discuss the elementary hydride transfer, hemiacetal formation, dehydration, and oligomerization and deprotonation elementary reaction steps within CHA in more detail. 


\section{$\underline{4.6 \text { Hydride Transfer }}$}

Under working conditions, the acid sites responsible for DMM formation are thought to be covered by methoxy intermediates and, as such, the reactions that lead to DMM formation are taken with respect to the bound methoxy. Hydride transfer then proceeds via the weak physisorption of methanol into $\mathrm{CHA}$ at $-19 \mathrm{~kJ} / \mathrm{mol}$ along with intrinsic activation for hydride transfer between the physisorbed methanol and the bound $\mathrm{CH}_{3}(+)$ which results in an apparent energy barrier of $69 \mathrm{~kJ} / \mathrm{mol}$. The reaction proceeds by the activation of the $\mathrm{AlO}^{*}-\mathrm{CH}_{3}{ }^{*}$ bond together with $\mathrm{C}-\mathrm{H}$ activation of the bound methanol to form a hydride to stabilize the ensuing the $\mathrm{CH}_{3}(+)$ carbenium ion intermediate. The transition state which is shown in Figure 4.6.1 displays classic Sn2 reaction characteristics, with the $\mathrm{O}^{*}-\mathrm{C}^{+}-\mathrm{H}^{-}$angle at $167^{\circ}$, methyl carbocation in the trigonal planer sp2 hybridization, and geometric inversion upon $\mathrm{C}^{*--\mathrm{O}}$ activation and $\mathrm{C}^{*}---\mathrm{H}$ bond formation. In addition, the proton on the reacting methanol interacts with the zeolite framework to help stabilize the increase in positive charge accumulating on the ${ }^{+} \mathrm{H}_{2} \mathrm{C}-\mathrm{OH}$ via partial resonance to the stabile formaldehyde molecule.

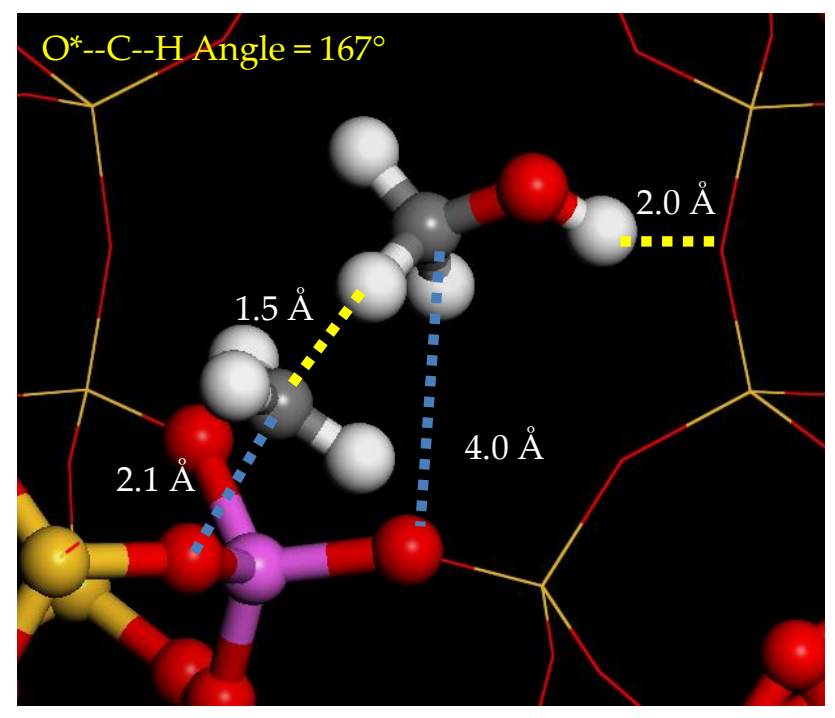

Figure 4.6.1: Transition state structure for hydride transfer between $\mathrm{CH}_{3}{ }^{+}$carbenium ion and physisorbed methanol.

The energy profile taken with respect to the (+)C--H(-) bond length shows the expected increase in energy with increasing $(+) \mathrm{C}--\mathrm{H}(-)$ bond length as the $\mathrm{CH}_{2} \mathrm{OH}(+)$ intermediate remains physisorbed due to the unstable nature of the charged carbon species (Figure 4.6.2). 
Upon the formation of methane, however, the $\mathrm{CH}_{2} \mathrm{OH}(+)$ intermediate adsorbs onto the $\mathrm{AlO}^{*}$ oxygen site.
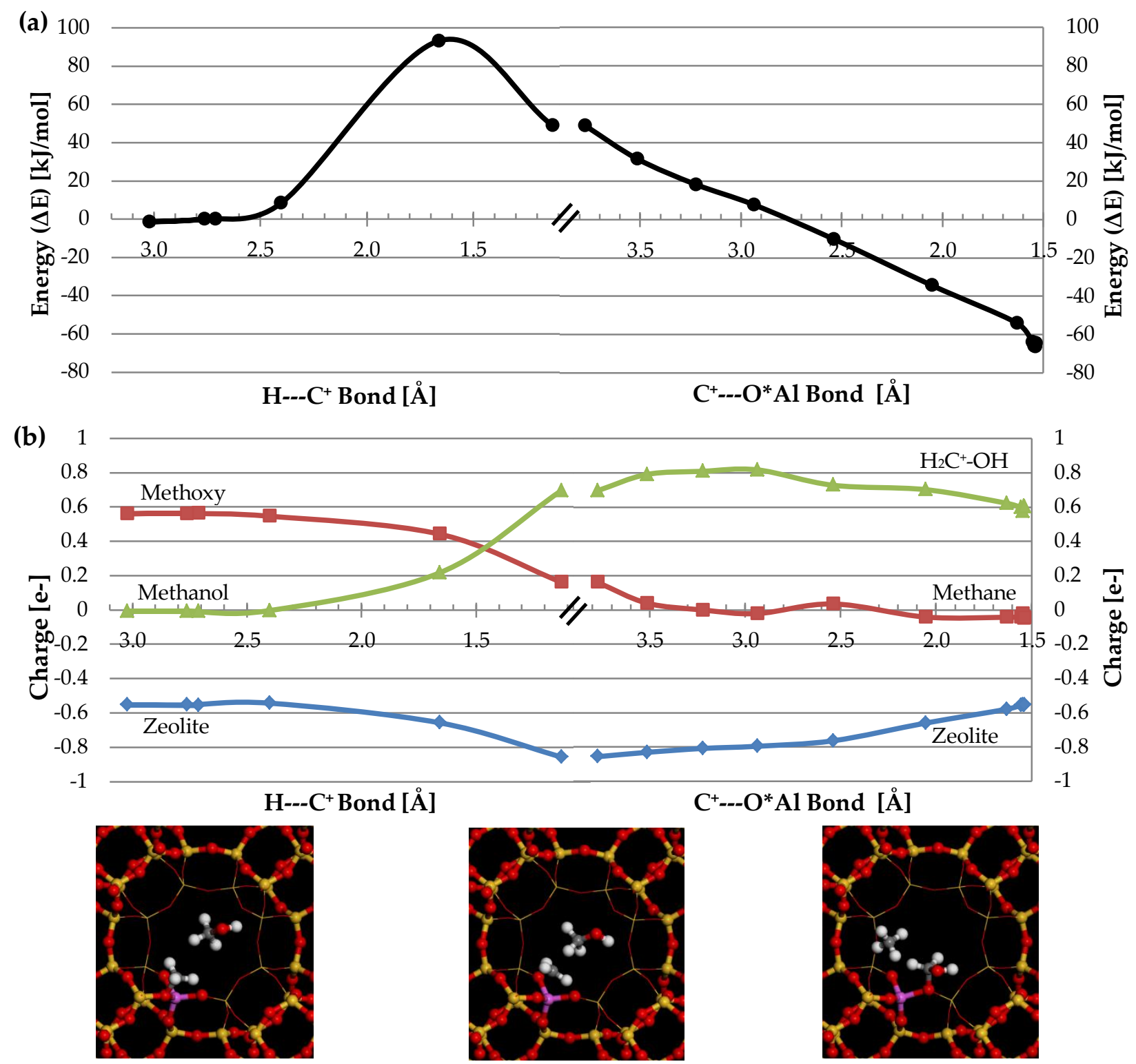

Figure 4.6.2: Energy profile and Bader charge analysis of summed charges on methyl (red), methanol (green), and zeolite (blue) measured across the $\mathrm{H}---\mathrm{C}^{*}$ coordinate during methanol hydride transfer, and across $\mathrm{C}^{*}--\mathrm{O}^{*} \mathrm{Al}$ coordinate during hydroxymethyl adsorption.

The energy progressively decreases from endothermic to exothermic ultimately resulting in a final apparent heat of reaction of $-85 \mathrm{~kJ} / \mathrm{mol}$. A Bader charge analysis along the 
reaction path shows a clear gain in positive charge from 0 to +0.7 accumulated on the hydroxymethyl intermediate in the transition state. This increase in charge on the $\mathrm{CH}_{2} \mathrm{OH}(+)$ species in the transition state is balanced by the gain of negative charge from -0.6 to -0.8 within the zeolite framework. As the ${ }^{+} \mathrm{H}_{2} \mathrm{C}-\mathrm{OH}$ intermediate adsorbs onto the $\mathrm{AlO}^{*}$ site, the zeolite site renormalizes back to -0.6 charge while the positive charge on the ${ }^{+} \mathrm{H}_{2} \mathrm{C}-\mathrm{OH}$ intermediate drops slightly from 0.7 to 0.6 ; the same charge observed for the adsorbed methoxy species at the reactant state. Following the hydride transfer, methane desorbs from the zeolite for $6 \mathrm{~kJ} / \mathrm{mol}$. This negligibly small energy results from methane's extremely limited interactions with the large CHA chamber.

The heats of reaction progress from exothermic to endothermic as the hydroxymethyl intermediate forms and adsorbs onto the $\mathrm{AlO}^{*}$ site is explained by the comparison of hydride affinity differences between gas phase methane and methanol. From gas phase calculations measured previously, the hydride affinity gain of methane outweighs the hydride affinity cost for methanol by $-283 \mathrm{~kJ} / \mathrm{mol}$. The hydride affinity gains outweigh the loss of energy associated with the somewhat weaker binding of the hydroxymethyl product $(660 \mathrm{~kJ} / \mathrm{mol})$ versus the methoxy reactant $(904 \mathrm{~kJ} / \mathrm{mol})$, thus resulting in the overall exothermicity for hydride transfer from physisorbed methanol to the bound methoxy in CHA.

\section{$\underline{4.7 \text { Hemiacetal Formation }}$}

The hydroxymethyl intermediate that forms can react with a second methanol molecule

to form the $\mathrm{HOCH}_{2}-\mathrm{O}(\mathrm{H})-\mathrm{CH}_{3}$ intermediate that deprotonates to form the hemiacetal. The methanol physisorbs near the bound hydroxymethyl intermediate resulting in an adsorption energy of $-29 \mathrm{~kJ} / \mathrm{mol}$. The binding energy is slightly higher than that for the adsorption of the $1^{\text {st }}$ methanol molecule as the negative $-\mathrm{OH}$ group of the adsorbed methanol binds to the positive carbon center of the $\mathrm{CH}_{2} \mathrm{OH}(+)$ intermediate (Figure 4.7.1). The reaction proceeds via the addition of the $-\mathrm{OH}$ from the physisorbed methanol to the $\mathrm{C}^{*}$ of the resulting $\mathrm{CH}_{2} \mathrm{OH}$ carbenium ion intermediate followed by the subsequent deprotonation of the positively charged $\mathrm{HOCH}_{2}-\mathrm{O}(\mathrm{H})-\mathrm{CH}_{3}$ intermediate to form the hemiacetal and regenerate the Brønsted acid site. The intrinsic energy barrier for this reaction was calculated to be $33 \mathrm{~kJ} / \mathrm{mol}$. The reaction 
proceeds via the backside attack of the oxygen on methanol onto the positively charged $\mathrm{CH}_{2} \mathrm{OH}(+)$ carbon center, where the transition state structure shows hydroxymethyl carbon in a sp2 orientation and a ${ }^{*} \mathrm{O}--\mathrm{C}--\mathrm{O}$ angle of $151^{\circ}$. The transition state also shows a tri-coordinated orientation, in which the negatively charged methanol oxygen is equidistant to the hydroxymethyl's positively charged carbon and adjacent hydrogen at $2.3 \AA$. Comparison to the second hydrogen at the same carbon center yields $2.6 \AA$; a $0.3 \AA$ increase.

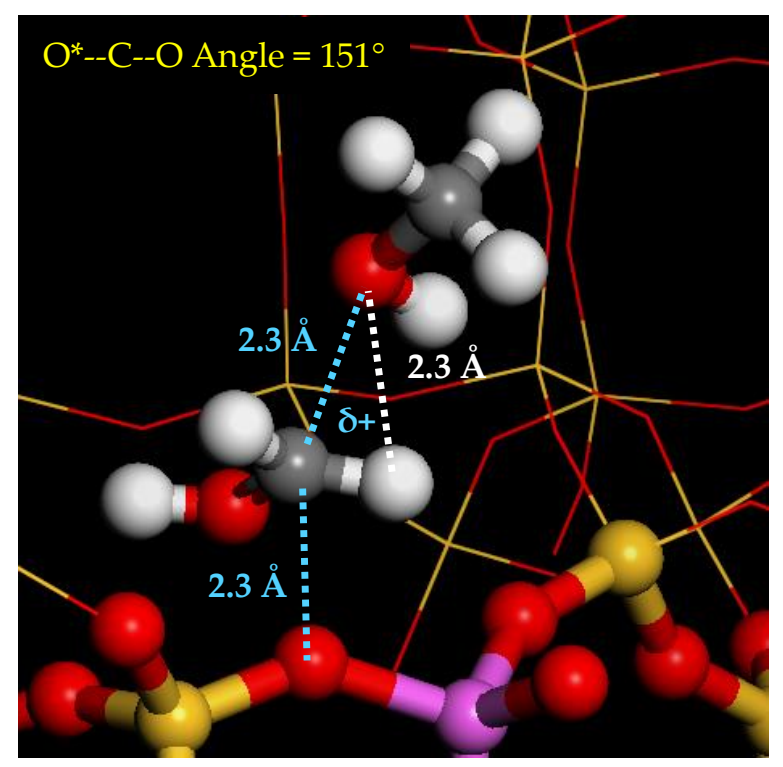

Figure 4.7.1: Transition state structure for $C^{*}$---O bond formation between hydroxymethyl cation and physisorbed methanol.

Bader charge analysis shows separation of charge during hemiacetal formation that yields the positively charged hydroxymethyl carbenium ion that lies between both the negatively charged oxygen of the zeolite and the negatively charged oxygen of the reacting methanol upon the heterolytic activation of the $\mathrm{C}^{*}$--O bond (Figure 4.7.2). A comparison of the charges on the reacting intermediates to the energy profile along the reaction coordinate shows that the highest point in energy activation matches the point in the charge analysis where the hydroxymethyl has the most positive charge. Interestingly, the methanol exhibits minor charge transfer, increasing from 0 to +0.06 at the transition state, whereas the $\mathrm{CH}_{2} \mathrm{OH}(+)$ cation increases from +0.56 to +0.67 and the zeolite negative charge changes from -0.56 to -0.73 from 
the reactant state to the transition state. This suggests that the transition state coincides with the heterolytic activation of $\mathrm{AlO}^{*}-\mathrm{CH}_{2} \mathrm{OH}$ to form the hydroxymethyl intermediate, with the physisorbed methanol experiencing minimal charge transfer until the subsequent $\mathrm{C}^{*}-\mathrm{O}$ bond formation between the hydroxymethyl cation and physisorbed methanol following the transition state.
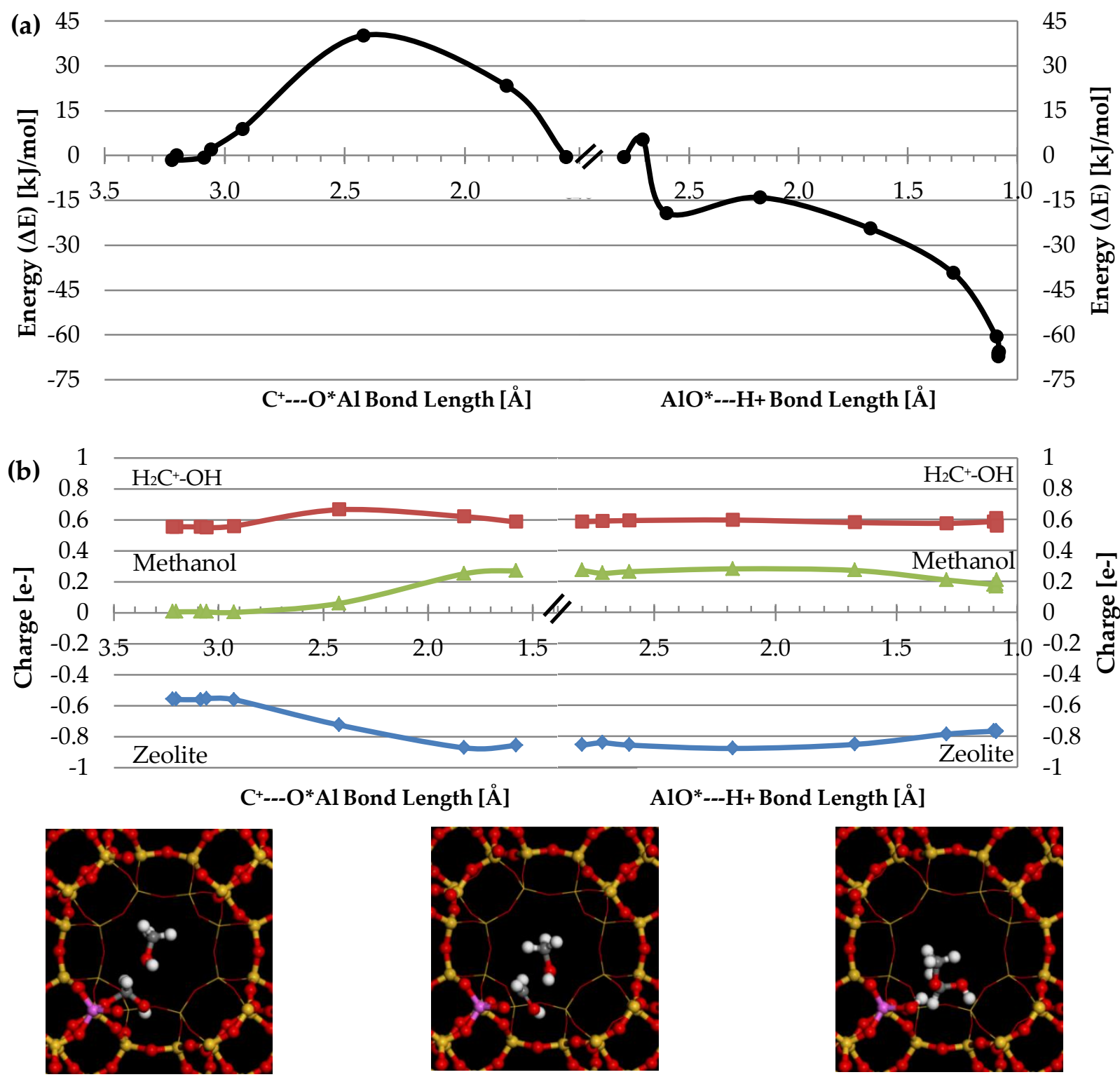

Figure 4.7.2: (a) Energy profile and (b) Bader charge analysis of hemiacetal formation, with summed charges on hydroxymethyl cation (red), methanol (green), and zeolite (blue) measured

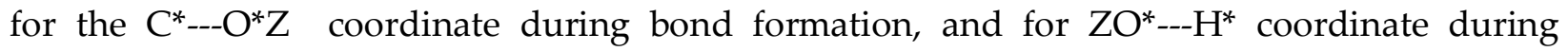
deprotonation to form the hemiacetal intermediate. 
Additional peaks appear along the reaction energy profile after the initial formation of the $\mathrm{C}^{*}-\mathrm{O}$ bond that correspond to the rotation and subsequent deprotonation of the $\mathrm{O}-\mathrm{H}$ group of the $\mathrm{HOCH}_{2}-\mathrm{O}(\mathrm{H})-\mathrm{CH}_{3}$ carbenium ion intermediate to form the hemiacetal product. These steps are all significantly lower in energy and downhill from the C-O bond formation step (Figure 4.7.2). As such, the rotation and deprotonation are inconsequential to the energetic barrier of the hemiacetal elementary reaction.

The charge analysis for the $\mathrm{C}^{*}-\mathrm{O}$ activation shows that the zeolite framework unexpectedly loses only 0.1 e- negative charge upon adsorbing the proton to the catalytic $\mathrm{O}^{*}$ site and still remains fairly negative at 0.8 e- total charge at the product state, in comparison to the adsorbed state with -0.6 e-. Analyzing the bond lengths at the product state, the $\mathrm{O}---\mathrm{H}^{+}$bond length between the hemiacetal and adsorbed proton is $1.38 \AA$; a distance well within possible hydrogen-bonding. Therefore, the minute changes in accumulated charge at the product state are due to the continued interaction between the hemiacetal and adsorbed proton, as the positive charge continues to interact with the hemiacetal intermediate.

\subsection{Dehydration}

The protonation and dehydration of the hemiacetal that forms occurs in a single concerted step. As the primary hydroxyl group of the $\mathrm{HO}-\mathrm{CH}_{2} \mathrm{OCH}_{3}$ is protonated, the terminal $\mathrm{H}_{2} \mathrm{O}--\mathrm{C}^{*}$ bond activates with an intrinsic activation energy of $88 \mathrm{~kJ} / \mathrm{mol}$. The primary mode for the transition state is the elongation of the $\mathrm{H}_{2} \mathrm{O}-\mathrm{C}^{*}$ bond forming water and methoxymethyl intermediate with sp2 planar configuration, where both the resulting water and the $\mathrm{CH}_{3} \mathrm{OCH}_{2}$ species that form interacts with neighboring $\mathrm{AlO}^{*}$ sites (Figure 4.8.1). The water that forms interacts with the $\mathrm{AlO}^{*}$ site via H-bonding during the reaction, whereas the cation carbon center of the methoxymethyl cation sits above the second neighboring $\mathrm{AlO}^{*}$ site, though the interaction is likely minimal due to the increased distance between them at $3.0 \AA$. This orientation also allows for $\mathrm{H}_{2} \mathrm{O}-\mathrm{C}^{*}$ bond activation to occur parallel to the two $\mathrm{O}^{*}$ sites. The reaction ends with water separating from the methoxymethyl intermediate and the methoxymethyl cation absorbing onto the neighboring $\mathrm{AlO}^{*}$ site, stabilizing the newly formed carbon center with an intrinsic heat of reaction of $23 \mathrm{~kJ} / \mathrm{mol}$. Water desorption from the zeolite 
is more expensive than methane at $38 \mathrm{~kJ} / \mathrm{mol}$, which likely derives from the additional cost of breaking polar interactions with the zeolite framework.

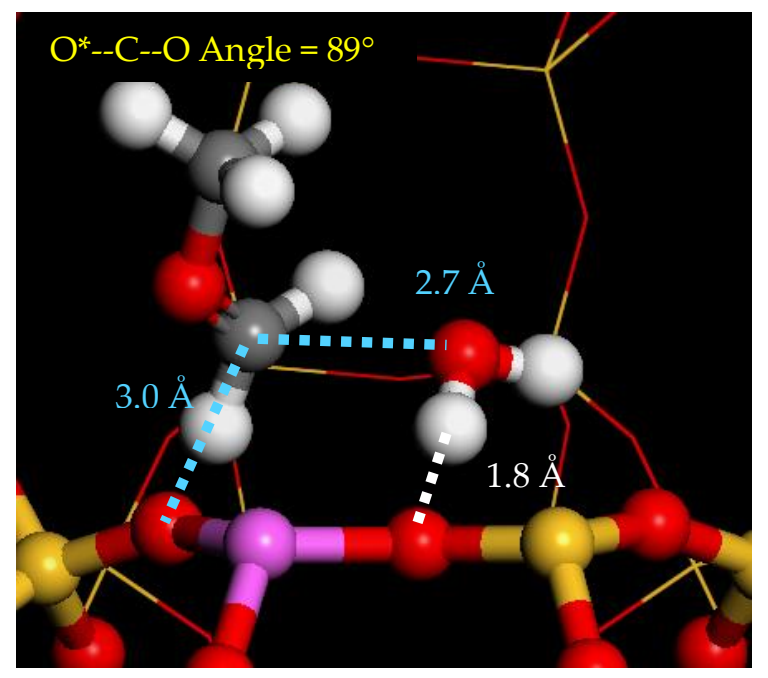

Figure 4.8.1: Transition state for dehydration of the hemiacetal to form water and methoxymethyl carbenium ion.

The Bader charge results in Figure 4.8.2b show an increase in the positive charge on the methoxymethyl intermediate while the water molecule charge drops from +0.2 to 0 as the reaction proceeds to the full formation of water and the methoxymethyl carbenium ion. The zeolite framework only gains slight negative charge, which mirrors the same slight negative charge loss during the deprotonation from the previous hemiacetal step. The proton at the dehydration transition state still has an $\mathrm{O}^{*}---\mathrm{H}$ bond length of $1.8 \AA$ which is sufficiently close enough for continued secondary interactions that further stabilize the $\mathrm{C}^{*}$---O cleavage transition state.

Just as was seen in hemiacetal formation, the highest point of positive charge accumulated on the methoxymethyl lines up with the peak of the energy barrier for dehydration (Figure 4.8.2), and coincides with the formation of the unstable $\mathrm{CH}_{3} \mathrm{OCH}_{2}(+)$ carbenium ion species. Following the transition state, the water remains charge neutral while the positive and negative charges on the methoxymethyl and zeolite framework decrease respectively as the intermediate adsorbs onto the $\mathrm{AlO}^{*}$ site. The zeolite framework returns to - 
0.6 charge, which was the same as for the adsorbed methoxy and adsorbed hydroxymethyl resting states.
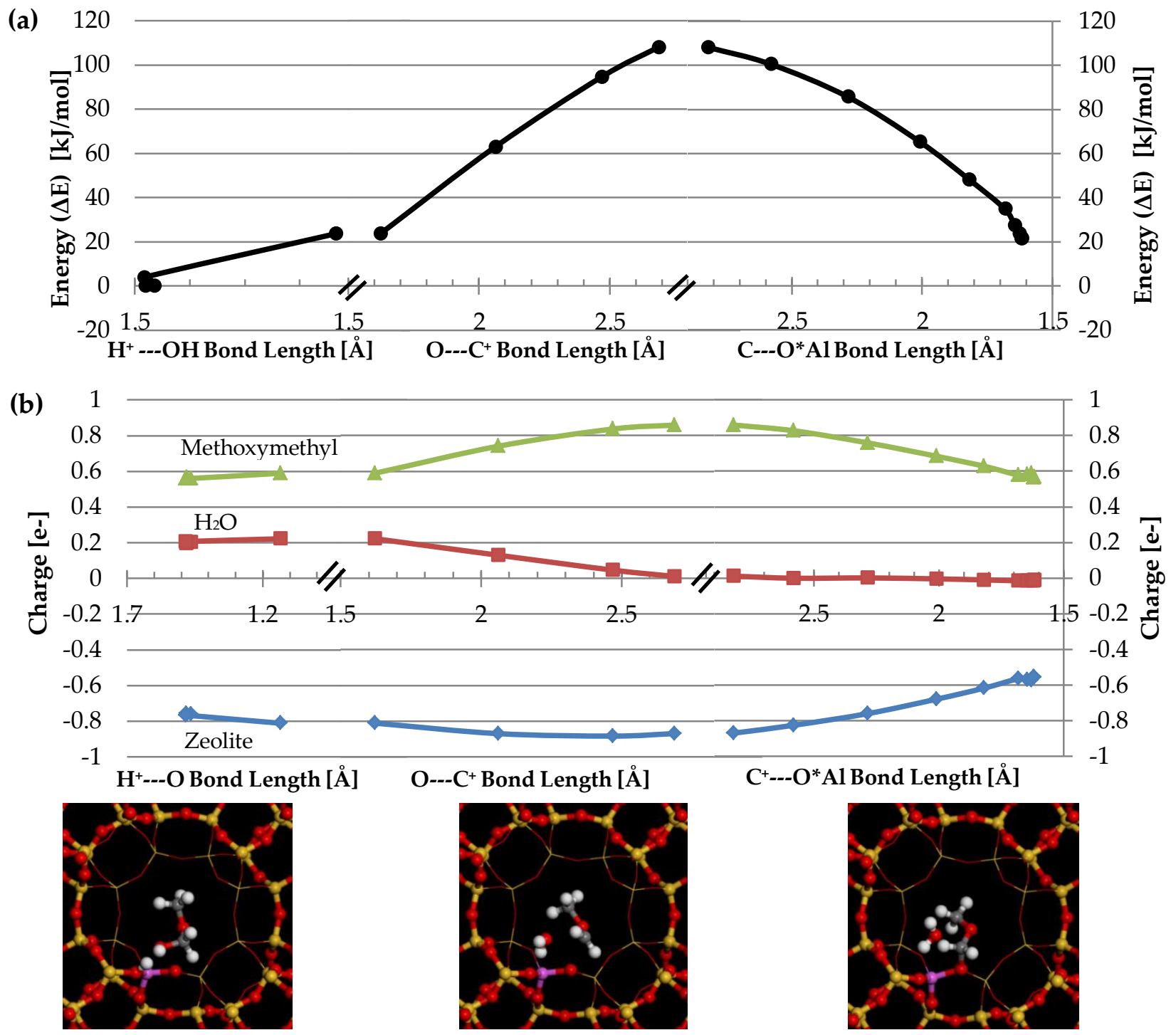

Figure 4.8.2: (a) Energy profile and (b) Bader charge analysis of hemiacetal dehydration, with methoxymethyl (green), water (red), and zeolite fragments (blue) measured for the $\mathrm{H}^{*}$---O coordinate during protonation, $\mathrm{O}---\mathrm{C}^{*}$ coordinate during dehydration, and $\mathrm{C}^{*}---\mathrm{O}^{*} \mathrm{Al}$ coordinate for methoxymethyl adsorption.

As noted in the previous step involving hemiacetal formation, the product state shows possible hydrogen bonding between the water and adsorbed methoxymethyl intermediate. 
While the Bader charge analysis does not show an exchange of charge between the water and methoxymethyl intermediate, the $\mathrm{O}---\mathrm{H}$ bond length between the water's oxygen and methoxymethyl's hydrogen is $2.4 \AA$, which lies just within range for hydrogen bonding. Furthermore, due to the accumulation of positive charge as shown from the Bader charge analysis, this distance is likely more than enough for secondary interactions that will sufficiently affect the desorption energy cost for the water molecule from the CHA zeolite.

\section{$\underline{4.9}$ Oligomerization and Deprotonation}

Following hemiacetal dehydration, the reaction continues with the adsorption of the final methanol reactant at $-38 \mathrm{~kJ} / \mathrm{mol}$ yields, and is $9 \mathrm{~kJ} / \mathrm{mol}$ stronger than that for the adsorption of the $2^{\text {nd }}$ methanol molecule $(-29 \mathrm{~kJ} / \mathrm{mol})$ and $19 \mathrm{~kJ} / \mathrm{mol}$ stronger than that for the adsorption of the first methanol molecule $(-19 \mathrm{~kJ} / \mathrm{mol})$. Similar to the second methanol adsorption, this increase in adsorption energy likely derives from the $\mathrm{O}---\mathrm{H}$ interaction between the hydroxyl group of methanol and the adsorbed methoxymethyl. The reaction proceeds via the activation of the $\mathrm{AlO}^{*}-\mathrm{C}$ bond between the bound methoxymethyl intermediate and $\mathrm{AlO}^{*}$ site, to form the sp2 planar methoxymethyl carbenium ion. The positive charge on the $\mathrm{CH}_{2}$ carbon center is stabilized by its interaction with the negatively charged $\mathrm{AlO}^{*}$ of the zeolite and the weakly nucleophilic oxygen of the physisorbed methanol. There is a geometric inversion at the planar

carbenium ion carbon center as it reacts with the physisorbed methanol to form the $\mathrm{C}^{*---\mathrm{O}}$ bond, resulting in an intrinsic energy barrier of $18 \mathrm{~kJ} / \mathrm{mol}$ (Figure 4.9.1). The resulting $\mathrm{CH}_{3} \mathrm{OCH}_{2}-\mathrm{O}(\mathrm{H}) \mathrm{CH}_{3}(+)$ coupled intermediate readily deprotonates to reform the Brønsted acid site and form the DMM product with a heat of reaction at $-54 \mathrm{~kJ} / \mathrm{mol}$. The energy profile for the overall reaction shown in Figure 4.8.2 indicates that there is no barrier for the subsequent deprotonation as the energies continue downhill to the DMM product state. Following deprotonation, the DMM product desorbs from the zeolite with a desorption energy of 99 $\mathrm{kJ} / \mathrm{mol}$; a significantly larger cost due to the product's size and hydrogen bonding to the newly formed Brønsted acid site. 


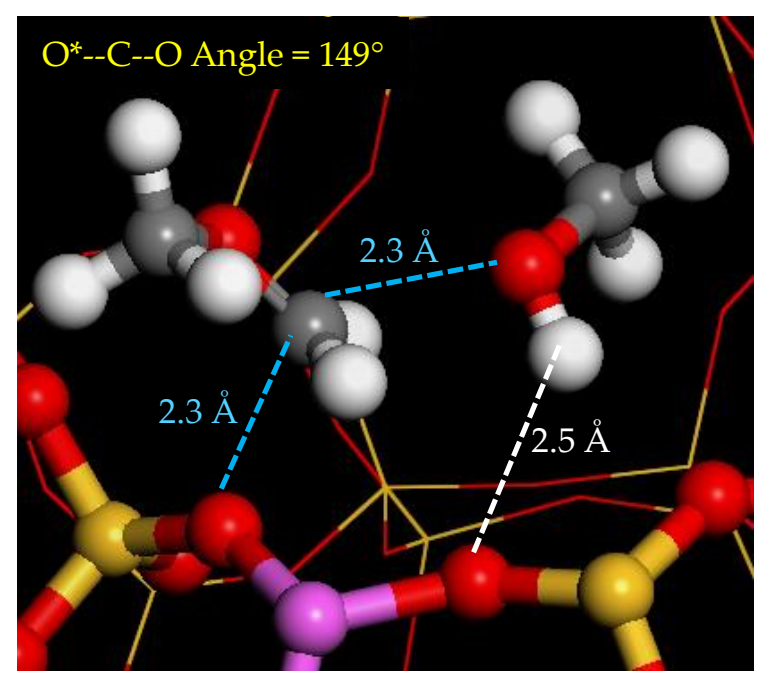

Figure 4.9.1: Transition state for DMM formation via $\mathrm{C}^{*}$---O bond formation and deprotonation.

The low intrinsic activation barrier for the $\mathrm{C}^{*--} \mathrm{O}$ bond formation between the methoxymethyl intermediate and physisorbed methanol derives from the secondary interactions between the transition state complex with the zeolite lattice as well as the stabilization of the positive charge on the carbenium ion that forms in the transition state between the negatively charged zeolite lattice and the weakly nucleophilic oxygen on the physisorbed methanol reactant. The non-bonding electrons on the oxygen in the methoxymethyl ion stabilize and dissipate the positive charge in the carbenium ion. The zeolite further stabilizes the transition state through secondary interactions localized near the $\mathrm{AlO}^{*}$ sites, as is evident by the $2.3 \AA$ distance between the $\mathrm{AlO}^{*}$ site and the positive carbon center of the methoxymethyl intermediate.

A Bader charge analysis shows that the methoxymethyl intermediate only gains +0.1 charge throughout the elementary reaction (Figure 4.9.2). The charge analysis also reveals that the positive charge is distributed to the methanol reactant even before reaching the transition state, confirming strong interactions between the methoxymethyl cation and the physisorbed methanol in $\mathrm{C}^{*}-\mathrm{O}$ bond formation. This continues into the transition state, with the highest point along the energy profile coinciding with highest amount of combined summed positive charge accumulated on both the methanol and methoxymethyl ion with a charge total of +0.88 . 

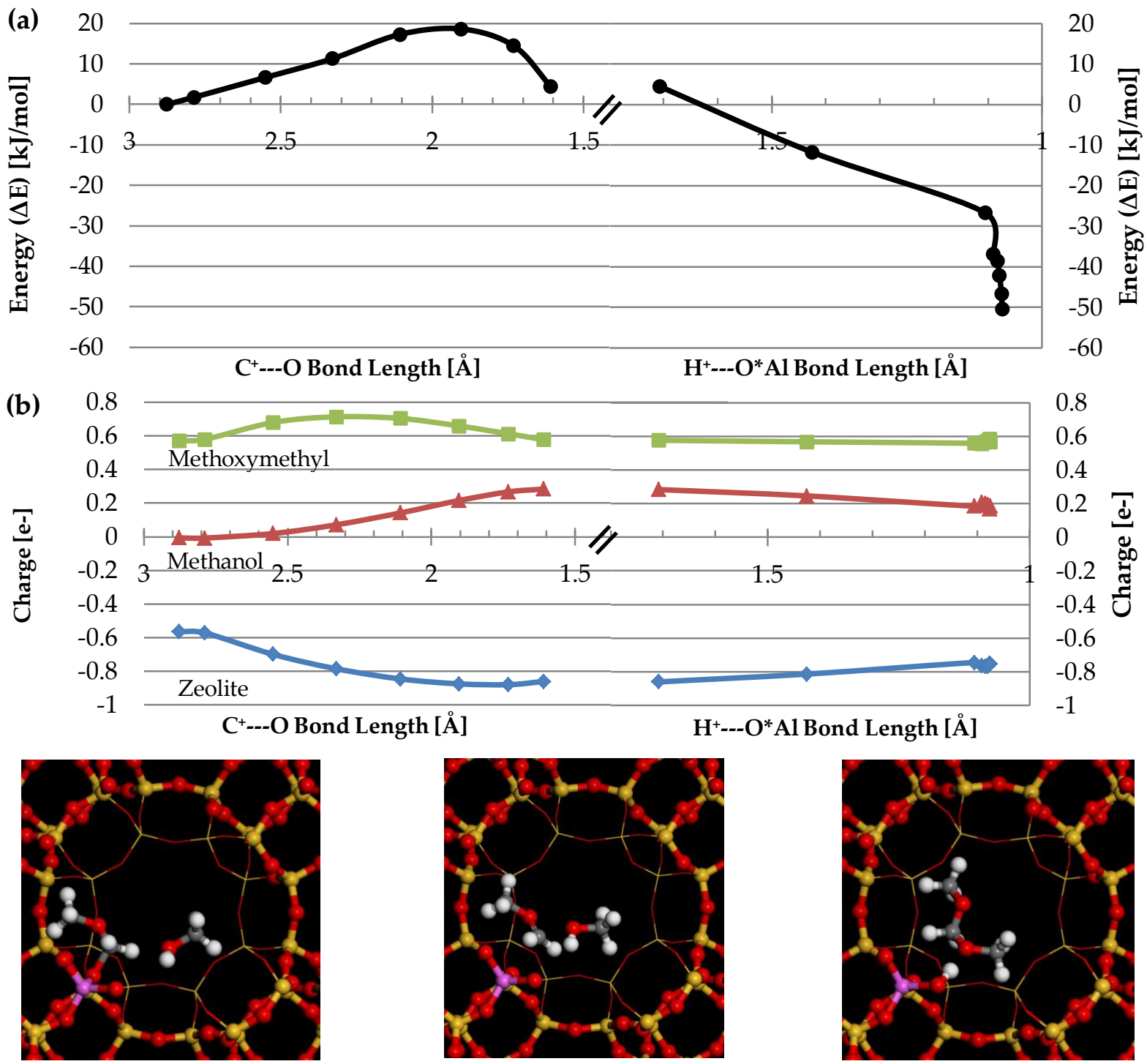

Figure 4.9.2: (a) Energy profile and (b) Bader charge analysis of oligomerization and deprotonation, with methoxymethyl (green), water (red), and zeolite fragments (blue) measured for the $\mathrm{C}^{*---\mathrm{O}}$ coordinate during oligomerization and $\mathrm{H}^{+}---\mathrm{O}^{*} \mathrm{Al}$ coordinate during deprotonation.

The positive charge that is transferred to the physisorbed methanol during reaction accumulates on the proton of the methanol, which lies within $2.5 \AA$ to the neighboring $\mathrm{AlO}^{*}$ site at the transition state. This is sufficiently close to maintain weak hydrogen bonding with the neighboring $\mathrm{AlO}^{*}$ site and assist in stabilization of charge at the cation intermediate. The close 
location of the proton to the $\mathrm{AlO}^{*}$ site along with the significant positive charge on the proton explains the ease of deprotonation that follows oligomerization. There is only a minor degree of charge transfer $(+0.1)$ observed between the separating DMM molecule and zeolite framework. This illustrates the near concerted nature of a two-step oligomerization/deprotonation process, where the major electronic effects occur during oligomerization and only minor changes in accumulated charge occur following the transition state during the deprotonation event. The DMM final product still maintains a total positive charge of +0.08 due to close proximity to the newly formed Brønsted acid site at $1.4 \AA$.

\subsection{Apparent Energies of Activation for DMM Formation in CHA}

The intrinsic activation energies, along with the overall reaction energies for the 4 major reaction steps (hydride transfer, hemiacetal formation, dehydration, and DMM formation) involved the reaction of methanol to DMM were used to construct the overall reaction energy profile depicted in Figure 4.10.1. The energies reported in the overall energy paths depicted in Figure 4.10.1 are taken with respect to the adsorbed methoxy reactant state and gas phase methanol molecule. The most enthalpically limiting step involves the first hydride transfer between $\mathrm{CH}_{3}(+)$ carbenium ion and physisorbed methanol to form methane and the $\mathrm{CH}_{2} \mathrm{OH}(+)$ intermediate. The high exothermic heats of reaction for the reactions of hydride transfer, hemiacetal formation, and DMM formation coupled with gains in energy from the subsequent adsorption of methanol reveal that the energetic profile proceeds downhill following the apparent hydride transfer barrier at $69 \mathrm{~kJ} / \mathrm{mol}$, taken with respect to the adsorbed methoxy reactant state. Even ignoring the gains in energy resulting from three methanol physisorptions at $-19 \mathrm{~kJ} / \mathrm{mol},-29 \mathrm{~kJ} / \mathrm{mol},-38 \mathrm{~kJ} / \mathrm{mol}$, respectively, the significant exothermic heats of reaction from all elementary steps, except for endothermic dehydration, supress all later elementary step apparent barriers to be lower than that for hydride transfer and making the reverse barrier for hydride transfer signifacntly lower than the forward barrier for hemiacetal formation.

The high apparent activation energy for hydride transfer reflects the significant difficulty associated with $\mathrm{AlO}^{*}-\mathrm{C}^{*}$ bond activation of the methoxy from the $\mathrm{AlO}^{*}$ site to form a methyl carbenium ion transition state in comparison to the larger delocalized charged 
intermediate species involved in the subsequent reactions. The methyl cation in the hydride transfer elementary step is the only non-oxygenate intermediate throughout the DMM formation mechanism, as all other intermediates are oxygenates of various length. The oxygen lone pair greatly stabilizes the localized positive charge of each charged oxygenate intermdiate during $\mathrm{AlO}^{*}-\mathrm{C}^{*}$ activation off of the $\mathrm{AlO}^{*}$ site. Calculated gas phase $\mathrm{AlO}^{*}-\mathrm{C}^{*}$ activation energies further illustrate differences in inherent cation stability, as the $\mathrm{AlO}^{*}-\mathrm{C}^{*}$ gas phase energy for methyl cation formation was $244 \mathrm{~kJ} / \mathrm{mol}$ higher than that for hydroxymethyl formation, and $292 \mathrm{~kJ} / \mathrm{mol}$ higher than methoxymethyl cation formation. This is testament to the low delocalization potential of the methyl cation center, and suggests that hydride transfer is the most enthalpically difficult elementary step for DMM formation in CHA.

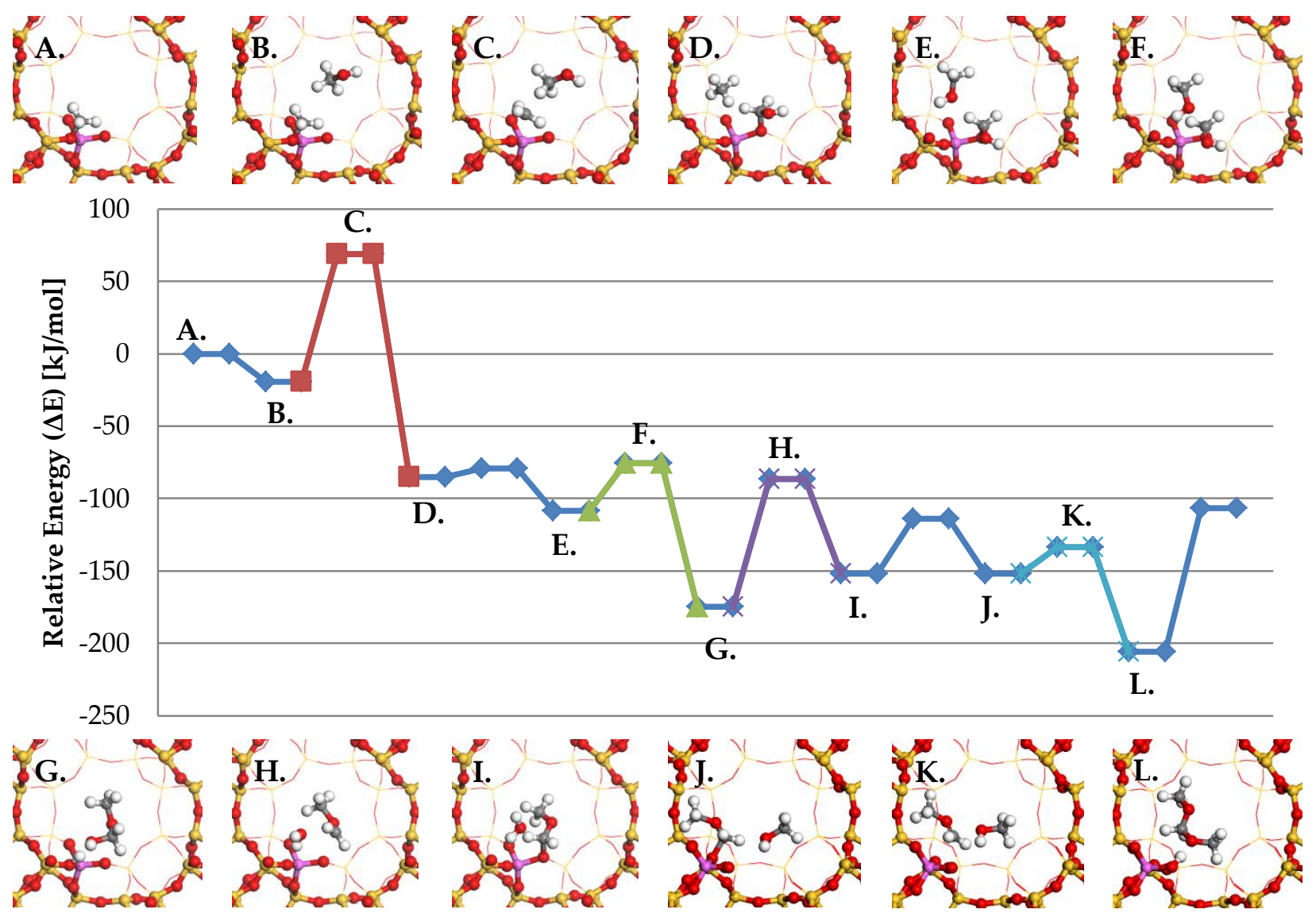

Figure 4.10.1: Energy diagram of DMM formation reaction referenced to the adsorbed methoxy reactant state illustrating apparent activation energies for methoxy-methanol hydride transfer (A-C), hemiacetal formation (E-G), dehydration (G-I), and DMM formation (J-L). 
The influence of the zeolite in solvating the transition states was analyzed by examining the vdW radii overlap between the transition states and the zeolite chamber. The results indicate that there are only localized interaction between the zeolite framework immediately surrounding the $\mathrm{AlO}^{*}$ sites (Figure 4.10.2). The transition state complexes sit well outside the majority of vdW radii of the surrounding CHA chamber "walls". The only overlap with the atoms in the transition state involve the atoms in the $\mathrm{SiO}_{2}$ framework that immediately surrounds the $\mathrm{AlO}^{*}$ site which stabilize the carbenium ion intermediates and hydrogen bond to the hydroxyl protons on physisorbed methanol. Even in the formed DMM product, the majority of close-contact interactions are localized around the $\mathrm{AlO}^{*}$ sites with only minimal interaction between the CHA chamber wall and the far methyl fragment furthest from the catalyst site. Therefore, the effect of catalyst likely results in the effect on the charged cation species that desorb from the $\mathrm{AlO}^{*}$ site, with only minor effects on the physisorbed molecules due to the large differences in size between the large chamber and small reacting molecules.
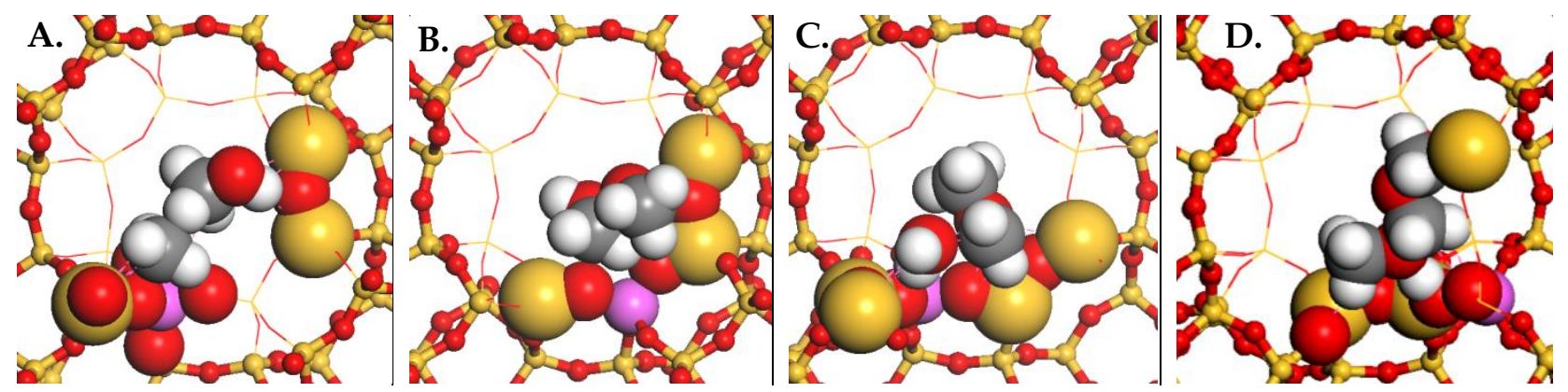

Figure 4.10.2: Illustration of reduced vdW spheres of close-contact CHA framework atoms and

transition state structures for (a) methoxy-methanol hydride transfer, (b) hemiacetal formation, (c) dehydration, and (d) oligomerization for DMM formation.

\section{$\underline{4.11 \text { Concluding Remarks }}$}

First principle density functional theory calculations show that methanol can react catalytically within CHA to form dimethoxymethane (DMM). The reaction appears to proceed via hydride transfer from methanol to a bound surface methoxy intermediate to form hydroxymethyl intermediate, the subsequent reaction of hydroxymethyl intermediate with a second physisorbed methanol molecule to form the $\mathrm{CH}_{3} \mathrm{OCH}_{2} \mathrm{OH}$ hemiacetal, the dehydration 
of the hemiacetal to form the methoxymethyl intermediate, and the oligomerization of the methoxymethyl intermediate and a third methanol to form DMM. In each of the elementary steps, the resulting carbenium ion that forms via the activation of the $\mathrm{AlO}^{*}-\mathrm{C}^{*}$ bond for alkoxy bound intermediate is planar and sp2 in character, thus highlighting the importance of cation stability for the overall reaction. Comparisons of the accumulated positive charge on the carbenium ion illustrates that the relative stability inherent to the intermediate, with the hydroxymethyl and methoxymethyl intermediates possessing higher delocalization potential due to the lone pair on the oxygen that sit $\alpha$ to the cationic carbon center. The oxygenate cations show accumulation of positive charge at the transition state,whereas the methyl cation formed during hydride transfer did not accumulate additional positive charge. Instead, the charge profile shows an immediate transfer of positive charge through the methyl cation directly to the reacting methanol, illustrating the low delocalization potential of the methyl cation. The relatively low stability of the methyl cation is directly responsible for the large energetic barrier for hydride transfer as well as the steep reverse barrier, making hydride transfer the kinetically limiting step in the catalytic conversion of methanol to DMM.

The influence of the CHA zeolite on the reaction was found to be mostly localized at the $\mathrm{AlO}^{*}$ sites due to the large chamber size relative to the small reacting intermediates. Analysis of vdW overlap suggests that CHA stabilizes the cation that results in the activation of $\mathrm{AlO}^{*}-\mathrm{C}^{*}$ via weak interactions between the physisorbed molecules, cationic transition state and the local oxygens near the $\mathrm{AlO}^{*}$ site. The lack of interaction with the rest of the $\mathrm{CHA}$ chamber as well as the exothermic nature of the proposed mechanism suggests that the CHA zeolite has potential to accommodate further coupling reactions of much larger molecules before steric limitations of the ridged framework hinder their formation.

\section{$\underline{4.12}$ References}

[1] Wei, Z.; Chen, Y.; Li, J.; Guo, W.; Doug, M.; Qin, Z.; Wang, J.; Jiao, H.; Fan, W., “Stability and reactivity of intermediates of methanol related reactions and $\mathrm{C}-\mathrm{C}$ bond formation over H-ZSM-5 acidic catalyst: A computational analysis", J. Phys. Chem. C 2016, 120, 6075-6087. 
[2] Zhang, W.; Chu, Y.; Wei, Y.; Xiangfeng, Y.; Xu, S.; Huang, J.; Zhang, M.; Zheng, A.; Deng, F.; Liu, Z., “Influences of the confinement effect and acid strength of zeolite on the mechanism of Methanol-to-Olefins conversion over H-ZSM-5: A theoretical study of alkenes-based cycle", Micropor. Mesopor. Mat. 2016, 216-229.

[3] Ilias, S.; Bahn, A., "Mechanism of the catalytic conversion of methanol to hydrocarbons", ACS Catal. 2013, 3, 18-31.

[4] Catizzone, E; Aloise, A.; Migliori, M.; Giordano, G., "From 1-D to 3-D zeolite structures: Performance assessment in catalysis of vapour-phase methanol dehydration to DME", Micropor. Mesopor. Mat. 2017, 243, 102-111.

[5] Chen, D; Moljord, K.; Holmen, A., "A methanol to olefins review: Diffusion, coke formation and deactivation on SAPO type catalysts", Micropor. Mesopor. Mat. 2012, 164, 239-250.

[6] Müller, S.; Liu, Y.; Vishnuvarthan, M.; Sun, X.; Veen, A.; Haller, G.; Sanchez-Sanchez, M.; Lercher, J., "Coke formation and deactivation pathways on H-ZSM-5 in the conversion of methanol to olefins", J. Catal. 2015, 325, 48-59.

[7] Pérez-Uriate, P.; Ateka, A.; Gayubo, A.; Cordero-Lanzac, T.; Aguayo, A.; Bilbao, J., “Deactivation kinetics for the conversion of dimethyl ether to olefins over a HZSM-5 zeolite catalyst", Chem. Eng. J. 2017, 311, 367-377.

[8] Celik, F.; Kim, T.; Mlinar, A.; Bell, A., “An investigation into the mechanism and kinetics of dimethoxymethane carbonylation over FAU and MFI zeolites", J. Catal. 2010, 274, 150162.

[9] Celik, F.; Kim, T.; Bell, A., "Effect of zeolite framework type and Si/Al ratio on dimethoxymethane carbonylation", J. Catal., 2010, 270, 185-195.

[10] Kresse, G.; Furthmüller, J., "Efficient iterative schemes for ab initio total-energy calculations using a plane-wave basis set", Phys. Rev. B 1996, 54, 11169-11186.

[11] Grimme, S., "Semiempirical GGA-type density functional constructed with a long-range dispersion correction", J. Comp. Chem. 2006, 27, 1787-1799. 
[12] Audouze, C.; Jollet, F; Torrent, M.; Gonze, X., “Comparison between projector augmented-wave and ultrasound pseudopotential formalisms at the density-functional perturbation theory level", Phys. Rev. B 2008, 78, 035105.

[13] Tang, W.; Sanville, E.; Henkelman, G., "A grid-based Bader analysis algorithm without lattice bias", J. Phys-Condens. Mat. 2009, 21, 084204.

[14] Henkelman, G.; Uberuaga, B. P.; Jónsson, H., “A climbing image nudged elastic band method for finding saddle points and minimum energy paths", J. Chem. Phys. 2000, 113, 9901-9904.

[15] Henkelman, G.; Jónsson, H., "Improved tangent estimate in the nudged elastic band method for finding minimum energy paths and saddles points", J. Chem. Phys. 2000, 113, 9978-9985.Christensen, A.; Carter, E. A. Phys. Rev. B 1998, 58, 8050-8064.

[16] Dassault Systèmes BIOVIA, Materials Studio, 6.1, San Diego: Dassault Systèmes, 2014.

[17] Ch. Baerlocher and L.B. McCusker, Database of Zeolite Structures: http://www.izastructure.org/databases. 
CHAPTER 5: Conclusions on the Similarities Between Aldol Condensation, Alkene Oligomerization, and DMM Formation

The work discussed herein has focused on the formation of $\mathrm{C}-\mathrm{C}$ and $\mathrm{C}-\mathrm{O}$ bonds over weak Lewis acid-base systems as well as Brønsted acid systems to understand the mechanism that control such paths for the synthesis of chemical as well as fuel intermediates. We highlight the similarities as well as the differences between aldol condensation, alkene oligomerization, and pathways to form DMM. First-principle density functional theoretical calculations were carried out in order to establish the intrinsic activation barriers, reaction energies for the individual elementary steps, and to establish the apparent activation energies and overall reaction energies in order to compare with experimental results. In an effort to understand the critical factors that control the elementary reaction steps, we have carried out detailed energetic decomposition of the elementary steps into basic thermochemical factors and features that control each step. Bader charge analysis together with the analysis of the vdW and closecontact bonds reveal similar conclusions regarding the kinetically relevant features facilitating light oxygenate and olefin conversions over metal oxides or zeolites.

\section{$\underline{5.1 \text { Mechanistic Similarity }}$}

Base-catalyzed aldol condensation is characterized by the formation of a negatively charged intermediate species, namely the enolate ion, which is followed by $\mathrm{C}^{*}$ - $\mathrm{C}$ bond formation between the newly formed charged intermediate and a weakly electrophilic reactant, in this case an adsorbed aldehyde or ketone. Charge analysis of enolate formation reaction confirms the accumulation of negative charge on the enolate during deprotonation of the surface aldehyde.

Alkene oligomerization is characterized by the formation of a positively charged intermediate species, namely the carbenium ion, which undergoes $C^{*}-C$ bond formation between the newly formed charged intermediate and a weakly nucleophilic reactant, in this case a physisorbed alkene. Analysis of positive charge accumulated the alkoxy confirms the formation of the carbenium ion during $\mathrm{AlO}^{*}-\mathrm{C}^{*}$ bond activation. In addition, a charge analysis 
along the reaction coordinate shows a direct transfer of positive charge from the carbenium ion to the weakly nucleophilic alkene during $\mathrm{C}^{*}---\mathrm{C}$ bond formation.

The proposed mechanism for DMM formation consists of four elementary steps. Hydride transfer is marked by the activation of the $\mathrm{AlO}^{*}-\mathrm{C}^{*}$ bond to form a carbenium ion together with simultaneous activation of a $\mathrm{C}^{*}---\mathrm{H}$ bond of the physisorbed methanol to stabilize the carbenium ion. The resulting hydroxymethyl intermediate subsequently reacts with a second methanol molecule to form the $\mathrm{CH}_{3} \mathrm{OCH}_{2} \mathrm{OH}$ hemiacetal via the simultaneous activation of the $\mathrm{AlO}^{*}-\mathrm{C}^{*}$ bond to form the $\mathrm{CH}_{2} \mathrm{OH}(+)$ carbenium ion that is stabilized by the interaction with the weakly nucleophilic oxygen of the second physisorbed methanol. The hemiacetal subsequently dehydrates to form the methoxymethyl cation, which subsequently binds to the $\mathrm{AlO}^{*}$ active site. The final oligomerization reaction to form DMM begins with methoxymethyl carbenium ion formation via $\mathrm{AlO}^{*}-\mathrm{C}^{*}$ activation that is stabilized and forms a $\mathrm{C}^{*}$--O bond with a third physisorbed methanol. Bader charge analysis of each elementary step confirms the formation of positively charged intermediate species, and the transfer of charge to weakly nucleophilic methanol or the $\mathrm{AlO}^{*}$ conjugate base site.

When described in this manner, the similarities in mechanism of these seemingly unique reactions are clear as all 3 reaction mechanisms involve the formation of a charged intermediate, and subsequent bond formation to a weakly nucleophilic or electrophilic reactant.

\section{$\underline{5.2 \text { Heterolytic Activation of Charged Intermediates }}$}

The formation of the charged intermediate observed in aldol condensation, alkene oligomerization, and DMM formation proceeds via heterolytic bond activation, which proceeds with minimal charge to the acting catalyst. A charge analysis of aldol condensation showed a nearly equal charge transfer between the enolate ion and separating proton of 0.4 charge, with less than +0.1 charge lost to the surface via the adsorbing proton and no charge change within the second neighboring spectator aldehyde.

A charge analysis of t-butyl alkene oligomerization in TON, MFI, and MOR consistently displayed equal charge transfer between the separating t-butyl cation and $\mathrm{AlO}^{*}$ site during carbenium ion formation, with less than 0.1 charge lost to the surrounding $\mathrm{SiO}_{2}$ framework and 
0 charge transferred to the physisorbed alkene. It was not until after the t-butyl cation formed did the physisorbed alkene undergo charge transfer with the carbenium ion during $\mathrm{C}^{*}$ - $\mathrm{C}$ bond formation. This charge transfer profile that separates the formation of the cation from the subsequent $C^{*}-C$ bond formation signifies a possible shift in mechanism, in which the stable tertiary t-butyl does not carry out an electrophilic attack until it has fully formed the cation, thus resulting in a nearly sequential mechanism. This is rather different than the simultaneous cation formation and $\mathrm{C}^{*}-\mathrm{C}$ bond formation that occurs with ethyl and 2-propyl cation intermediates. A detailed charge analysis of smaller ethyl and 2-propoyl cations also suggested heterolytic splitting of the $\mathrm{AlO}^{*}-\mathrm{C}^{*}$ bond, as every case found the majority of charge to be localized at the $\mathrm{AlO}^{*}$ site with only minimal charge lost to the rest of the $\mathrm{SiO}_{2}$ framework.

A charge analysis of the first elementary step of DMM formation displays a charge transfer profile that nearly overlaps directly with ethyl carbenium ion activation from alkene oligomerization. In addition, the reverse hydroxymethyl adsorption during hydride transfer and the forward hydroxymethyl cation formation from hemiacetal formation both directly overlap with the 2-propyl cation formation profile. For each cation formation step in hydride transfer, there are two distinct heterolytic cleavage steps that directly overlap with those observed for alkene oligomerization. The $\mathrm{H}_{2} \mathrm{O}--\mathrm{C}^{*}$ cleavage that results in dehydration shows strong heterolytic cleavage characteristics, with the charge localized entirely on the separating cation species and resulting in a minimal change in the change on the zeolite until the methoxymethyl adsorption. Finally, the reactivation of the methoxymethyl for oligomerization to form the DMM product displays this same charge profile observed in the previous three elementary steps, and with the same characteristics described repeatedly in this section.

The data presented here provide strong evidence supporting the similar heterolytic nature of charged intermediate formation across these reactions and all tested catalysts.

\subsection{Delocalization and Steric Limitations of Electrophilic/Nucleophilic Reactant}

In both aldol condensation and alkene oligomerization, the $C^{*}-C$ bond formation step is directly influenced by the size of the carbon chain length as a result of delocalization potential 
over the chain length as well as steric repulsion induced via intermolecular interactions between carbon tails of the reacting intermediate species.

Comparison of various sized aldehydes for $C^{*}-C$ bond formation in aldol condensation displayed increasing intrinsic activations energies with increasing carbon chain length for both the enolate as well as the neighboring aldehyde. The coupling of sp2 carbon centers forces the carbon tails into close proximity with one another. This is reflected in close $\mathrm{C}^{*}-\mathrm{C}$ distances between different carbon chains as reported in the transition states for $\mathrm{C}^{*} \mathrm{C}$ bond formation. The close contact results in steric repulsion that increases the intrinsic activation energies associated with larger transition state complexes. In particular, the nucleophilic primary carbon on the ethanal enolate yields the lowest barriers for $C^{*---C}$ bond formation $(6 \mathrm{~kJ} / \mathrm{mol}-14 \mathrm{~kJ} / \mathrm{mol})$ in comparison to the secondary nucleophilic carbon centers on propanal and butanal enolates. The influence of steric repulsion is further emphasized in the comparison of propanal selfcoupling to propanal-acetone cross-coupling, in which the tertiary carbon center of acetone increased the calculated intrinsic energy barrier by $28 \mathrm{~kJ} / \mathrm{mol}$ in comparison to the secondary carbon center of propanal. Bader charge analysis comparison of acetone to propanal suggests that acetone likely exhibits a slight benefit from higher accumulation of positive charge due to lower delocalization potential, but this effect is overtaken by the large intermolecular repulsion.

Similar conclusions were also reached with regards to $\mathrm{C}^{*}-\mathrm{C}$ bond formation in alkene oligomerization for carbenium ions and alkenes with varying degrees of substitution. Steric limitations were found to be important factors in the diminishing energetic returns calculated for gas phase $C^{*}-\mathrm{C}$ bond formation, as more substituted carbon centers would induce far larger repulsion than otherwise observed for secondary or primary hydrocarbon intermediates. This was also observed in the increased spacing between carbenium ion and alkene at the transition state for more substituted carbon centers, as the high steric repulsion would force the two reacting intermediates to lie further apart within the transition state complex. These effects, however, can also be explained by the increased stability of higher substituted carbon centers that would allow for larger distances between reacting carbon centers at the transition state. This study cannot fully conclude the effects of steric repulsion on $C^{*}-C$ bond formation for alkene oligomerization and requires further investigation through a more detailed energy 
decomposition analysis that would allow decoupling of attractive and repulsive secondary interactions that might be achieved through a force field model.

The activation barriers and kinetics are also largely influenced by the ability of the carbenium ion and the physisorbed alkene to delocalize positive charge that forms in the transition state. This was made apparent in the comparison of isolated positive charge calculated on gas phase molecules using Mulliken charge analysis which showed the lowest localized positive charge at the carbenium ion carbon center with highest degrees of substitution. The t-butyl cation yielded the lowest localized positive charge at +0.24 e- whereas the ethyl cation resulted in the highest at +0.63 e-. This influence of delocalization was also observed in the charge transfer profiles calculated along the reaction coordinate, with the less stable ethyl cation displaying immediate transfer of all positive charge to the reacting alkene due to the high instability of the primary carbenium ion center that forms in the ethyl cation. As a comparison, $\mathrm{t}$-butyl cation yielded a more gradual transfer of positive charge, where the positive charge was observed to accumulate on the carbenium ion during $\mathrm{AlO}-\mathrm{C}^{*}$ activation, and then finally transfer to the physisorbed alkene during $\mathrm{C}^{*}$ - $\mathrm{C}$ bond formation, thus illustrating the greater stability of $t$-butyl tertiary carbon center. It is therefore readily apparent that the differences in delocalization potential within the charged cation species directly affects the cation stability and further reactivity for $\mathrm{AlO}^{*}-\mathrm{C}^{*}$ activation necessary for alkene oligomerization.

In addition to the size and the degree of substitution of the reacting alkoxide, the size and substitution of the reacting alkene can also significantly influence the catalytic behavior. A detailed charge analysis for the reactions of propene verses those with isobutene provided important insights into the difference in accumulated charge at the resulting oligomer carbon center. The reaction of ethoxy-isobutene resulted in an accumulation of +0.25 at the oligomer carbon center whereas the reaction of ethoxy-propene yielded a charge accumulation of +0.36 . This quantifies the differences in potential stability provided to the reacting carbenium ion upon $C^{*}-C$ bond formation to the dimerized intermediate, and explains the higher activity of isobutene for alkene oligomerization in comparison to the less active propene that consistently yielded less favorable free energies of activation for all reacting pairs. Surprisingly, the Bader 
charge analysis profiles displayed a near perfect overlap between propene and isobutene, with only the total accumulated charge differing between the two alkenes. This was also observed for aldol condensation, where the neighboring aldehyde or ketone was found to have a negligible effect on both the energetics and charge transfer during enolate formation. This simply reflects that the alkene only affects the distribution of positive charge during $\mathrm{C}^{*}-\mathrm{C}$ bond formation, the start of which relies heavily upon the inherent stability of the carbenium ion.

Whether an aldehyde coupling to an enolate or an alkene oligomerizing with a carbenium ion, the data presented herein show that the bond formation steps of these reactions are determined by intermolecular steric effects between repulsive carbon tails and delocalization potential within resulting charged intermediates following $\mathrm{C}^{*}-\mathrm{C}$ bond formation.

\subsection{Transition State Structure and Energetic Barriers Associated with Ion Formation}

Alkene oligomerization and DMM formation display structurally similar transition states with planar or nearly-planer orientations during the formation of charged intermediates, while the transition states for enolate formation in aldol condensation result in more restricted orientations by comparison. A detailed analysis of the transition state structures shown in Figure 2.9.1 involved in the heterolytic activation of the $\mathrm{C} \alpha-\mathrm{H}$ bond of aldehydes and ketones to form the enolate intermediate along with a proton over the oxide surfaces proceed via the formation of a nearly planar $\mathrm{M}--\mathrm{O}=\mathrm{CH}-\mathrm{C}^{(\delta-)}(\mathrm{R})--\mathrm{H}^{(\delta+)}-\mathrm{O}$ transition state with an $\mathrm{M}-\mathrm{O}=\mathrm{C}$ bond angle that is on average enolate formation during aldol condensation displays nearly planar orientation, with an average angle of $158^{\circ}$ that is structurally limited by the $\mathrm{Zr}-\mathrm{O}^{*}$ bond between the oxygen of the enolate and the $\mathrm{Zr}^{*}$ metal site of the metal oxide surface. A detailed analysis of the different elementary steps involved in the aldol condensation and a comparison with the experimental results indicate that the enolate formation step as the rate determining step rather than the $C^{*}-C$ bond formation step. The bulkier enolates result in higher apparent free energies of activation, and more constricted angles due to steric limitations of the carbon backbone with the oxide surface that limits the $\mathrm{C}_{\alpha--\mathrm{H}}$ proton from leaning over towards the $\mathrm{O}^{*}$ site.

In the case of alkene oligomerization, the alkoxy sits directly between the negative $\mathrm{AlO}^{*}$ site and the nucleophilic $\pi$-bond of the alkene. The secondary interactions that take place in 
zeolites can aid in solvating the transition state complexes and allow for less constricted intermediate placement than observed on the metal oxide surface. The angle between the $\mathrm{AlO}^{*}$ site, carbenium ion carbon center, and physisorbed alkene $\left(A l O^{*}-R_{1}(\delta+)-R_{2}\right)$ is limited by the steric limitations of the zeolite framework on the physisorbed alkene above the carbenium ion, as well as the inherent stability of the carbenium ion. Alkene oligomerization transition states within MOR and MFI zeolites display roughly $170^{\circ}$ angles between the $\mathrm{AlO}^{*}$ site, carbenium ion carbon, and reacting carbon on the physisorbed alkene, with the carbenium ion at the sp2 planar orientation that undergoes geometric inversion upon $C^{*}-C$ bond formation with the alkene. Oligomerization transition states in TON show angles of $168^{\circ}$ to $118^{\circ}$ for ethyl to t-butyl carbenium ions. These are likely constrained by steric limitations of the zeolite framework with the reaction intermediates as suggested by close-contact analysis. Energetic decomposition from Born-Haber cycle analysis shows that the carbenium ion formation holds high significance to the overall energetic barrier associated with alkene oligomerization, and any additional stability provided by zeolite via secondary interactions lowers the overall energetic barrier.

A number of the elementary steps involved in the formation of DMM in CHA proceed via the formation of carbenium ion intermediates and display near- $180^{\circ} \mathrm{AlO}^{*}-\mathrm{R}_{1}(\delta+)--\mathrm{R}_{2}$ angles where $R_{1}(\delta+)$ is the formation of the carbenium ion and $R_{2}$ is the nucleophile that $R_{1}$ attacks. The angle for hydride transfer was calculated to be $167^{\circ}$ whereas that for hemiacetal formation is $151^{\circ}$. The transition state for hemiacetal formation partially resembles that for alkene oligomerization transition state, with the reacting methanol oxygen shared between the hydroxymethyl carbon and neighboring proton in a similar trigonal orientation reminiscent of alkene oligomerization. During the dehydration elementary step, the $\mathrm{H}_{2} \mathrm{O}-\mathrm{C}^{*}$ separation takes place parallel to the $\mathrm{AlO}^{*}$ sites with re-adsorption beginning just as dehydration completes. The last elementary step, oligomerization to form DMM final product, displays similar $149^{\circ}$ angle between the $\mathrm{AlO}^{*}$ site, methoxymethyl cation intermediate, and reacting hydroxyl on the physisorbed methanol. The angle is less than $180^{\circ}$ in order to maintain the $\mathrm{O}---\mathrm{H}$ interaction between the neighboring $\mathrm{AlO}^{*}$ site and methanol proton. As observed with dehydration, the $\mathrm{C}^{*}$---O bond formation and deprotonation during oligomerization to form DMM occur near simultaneously. There is observed very similar characteristics across all transition states in 
geometric configuration during DMM formation, with the cation intermediate orientated in a near sp2 planar configuration at the transition state that undergoes geometric inversion.

As was observed for aldol condensation and alkene oligomerization, gas phase energetic analysis of each elementary step for DMM formation shows cation formation to be the most energetically expensive process. Comparisons of charge transfer profiles with energetic profiles of each elementary step illustrate the point of highest energetic difficulty coincides with the largest accumulation of charge on the reacting cation as it is formed via $\mathrm{AlO}^{*}-\mathrm{C}^{*}$ activation or $\mathrm{H}_{2} \mathrm{O}^{*}-\mathrm{C}^{*}$ activation in the case of dehydration. In addition, the carbenium ion is shown to be stabilized via secondary interactions with the surrounding zeolite and physisorbed reacting molecule where possible

Aldol condensation, alkene oligomerization, and DMM formation reactions have striking similarities concerning the formation of charged intermediates in both transition state structure and energetic difficulty to the overall reaction. It is clear from these systems that the charged intermediate formation step is consistently the most difficult step and more demanding than the subsequent bond formation steps, regardless of reactant or catalyst.

\subsection{The Role of the Catalyst on Intermediate Stabilization}

Born-Haber cycle analysis of aldol condensation and alkene oligomerization yield the same conclusions regarding reactivity based on stabilizing interactions between the reacting intermediates and the catalyst at transition state. The analysis found a strong linear correlation between lower apparent free energies of activation and more favorable interaction energies.

In aldol condensation, this interaction energy was attributed to the coulombic interactions between intermediates based on localized charge strength, the proximity of the adsorbed proton and enolate on the metal oxide surface, and effects of the metal oxide surface on the adsorbed intermediates. While the delocalization potential of the enolate ion is inherent to the reactants, the metal oxide catalyst is responsible for both the proximity of the proton and enolate at transition state as well as the stabilization of the separating intermediates during heterolytic $\mathrm{C}^{*}$---H cleavage. Bader charge analysis reveals that the strength of the Lewis acid metal sites and Lewis base oxygen sites determine the amount of charge separated between the 
enolate and proton, with stronger site pairs allowing for greater separation of charge due to increased stability provided to the respective charged intermediates.

For alkene oligomerization, more favorable referenced interaction energies with the transition state were attributed to lower apparent free energies of activation with the same strong linear correlation identified for aldol condensation. In addition to the inherent carbenium ion stability determined by alkoxy substitution, smaller zeolite structures allowed for higher secondary interactions with the transition state by maximizing the catalytic stabilizing effects on the charged intermediates. These secondary effects of the zeolite on the charged intermediates are also influenced by the inherent stability of the carbenium, as less inherently stabilized carbenium ion such as ethyl cations showed far higher differences in referenced secondary energy between TON, MFI, and MOR than observed for t-butyl cation regarding catalytic differences. Unlike in the case of aldol condensation, Bader charge analysis showed almost no coulombic differences in the $\mathrm{AlO}^{*}--\mathrm{C}^{*}$ heterolytic split for differing zeolites, as the chemical make-up of all tested zeolites are all $\mathrm{SiO}_{2}$ with a single Al-site.

With regards to DMM formation in CHA, analysis of potential secondary interactions via close-contact analysis of transition state structures within the zeolite reveal the transition state to interact with the catalyst close to the $\mathrm{AlO}^{*}$ sites with only minimal secondary interactions to the hydroxyl group on physisorbed methanol. With regards to literary evidence such as "nest effect" introduced by Derouane describing on the effect of zeolite framework [1-3] and importance of catalytic interaction to free energies of activation, conclusions reached in similar systems and reactions heavily imply the potential for additional coupling reactions in CHA to form larger products that can deposit within the zeolite chamber and deactivate the catalyst.

It was already previously noted that the charged intermediate formation step yields the highest energetic barrier for aldol condensation, alkene oligomerization, and DMM formation. It therefore follows that these observed coulombic and secondary interactions between catalyst and reacting intermediates would facilitate charged species formation, and result in more favorable energies. Born-Haber cycle analysis of both aldol condensation and alkene oligomerization yield quantifiable evidence illustrating this effect, and its application to 
possible coupling reactions in CHA assists to further the understanding of deactivation in CHA type zeolite during methanol-to-olefin reactions.

\subsection{Application to Future Studies}

The work reported here analyzes and identifies the shared importance of charged intermediate stability, catalytic stabilization of transition states, and intermolecular and catalytic steric effects on the activity of heterogeneous catalysts for aldol condensation, alkene oligomerization, and DMM formation. Each of these reactions hold significant potential to the future of catalytic conversion of biomass derivatives through better understanding of features that facilitate these reactions as well as insights into deactivation routes that hinder these systems. In addition, the implications of these qualitative similarities of seemingly different systems illustrate an importance in the application of these conclusions towards future studies in the pursuit of light olefin and oxygenate upgrading. A computation study on methanol-toolefins reaction by Zhang et al. has already noted extremely similar conclusions to those reached here regarding importance on transition state stability by the zeolite framework [4]. The alkylation of isobutane by isobutene also concludes a familiar importance of carbenium ion stability by the physisorbed isobutene that facilitates alkylation in comparison to hydride transfer [5]. The conclusions reached here and their relevance across various heterogenous systems help to further the understandings of other oxygenate and olefin coupling reactions beyond what is studied here, and may help to accelerate the pursuit of catalytic conversion of biomass derivatives.

\subsection{References}

[1] Derouane, E., "Shape selectivity in catalysis by zeolites: The nest effect", J. Catal. 1986, $100,541-544$.

[2] Derouane, E.; André, J.; Lucas, A., "Surface curvature effects in physisorption and catalysis by microporous solids and molecular sieves", J. Catal. 1988, 110, 58-73. 
[3] Derouane, E.; André, J.; Lucas, A., “A simple van der Waals model for molecule-curved surface interactions in molecular-sized microporous solids", Chem. Phys. Let. 1987, 137, 336-340.

[4] Zhang, W.; Chu, Y.; Wei, Y.; Xiangfeng, Y.; Xu, S.; Huang, J.; Zhang, M.; Zheng, A.; Deng, F.; Liu, Z., “Influences of the confinement effect and acid strength of zeolite on the mechanism of Methanol-to-Olefins conversion over H-ZSM-5: A theoretical study of alkenes-based cycle", Micropor. Mesopor. Mat. 2016, 216-229.

[5] Janik, M.; Davis, R.; Neurock, M., “A density functional theory study of the alkylation of isobutene with butane over phosphotungstic acid", J. Catal. 2006, 244, 65-77. 